\title{
Ethyl Nitroacetate in Aza-Henry Addition on Trifluoromethyl Aldimines: A Solvent-Free Procedure To Obtain Chiral Trifluoromethyl $\alpha, \beta$-Diamino Esters
}

Luca Parise, Alessia Pelagalli, Lucio Pellacani, Fabio Sciubba, Maria Cecilia Vergari, and Stefania Fioravanti*

Dipartimento di Chimica, Università di Roma La Sapienza, P.le Aldo Moro 5, I-00185 Roma, Italy

E-mail: stefania.fioravanti@uniromal.it

Supporting Information

Computational details

${ }^{1} \mathrm{H},{ }^{13} \mathrm{C}$ and ${ }^{19} \mathrm{~F}$ NMR spectra of all new compounds

S3-S73

2D NMR spectra and optimized geometries to determine

the absolute configuration of the new chiral centers

S74-S89 


\section{Computational details}

All optimized geometries were located using hybrid functional theory (B3LYP) ${ }^{1}$ and the 6$31 \mathrm{G}(\mathrm{d}, \mathrm{p})^{2}$ basis set using the continuum solvation model $^{3}$ with chloroform $(\varepsilon=4.71)$ as the solvent conforming to the experimental conditions. All calculations were carried out using the Gaussian 09 program. ${ }^{4}$

\footnotetext{
${ }^{1}$ Becke, A. D. J. Chem. Phys. 1993, 98, 1372-1377.
}

2 (a) Ditchfield, R.; Hehre, W. J.; Pople, J. A. J. Chem. Phys. 1971, 54, 724-728. b) Hehre, W. J.; Ditchfield, R.; Pople, J. A. J. Chem. Phys. 1972, 56, 2257-2261. c) Hariharan, P. C.; Pople, J. A. Theor. Chim. Acta 1973, 28, 213-223.

${ }^{3}$ Barone, V.; Cossi, M.; Tomasi, J. J. Comput. Chem., 1998, 19, 404-17.

${ }^{4}$ Gaussian 09, Revision D.01, Frisch, M. J.; Trucks, G. W.; Schlegel, H. B.; Scuseria, G. E.; Robb, M. A.; Cheeseman, J. R.; Scalmani, G.; Barone, V.; Mennucci, B.; Petersson, G. A.; Nakatsuji, H.; Caricato, M.; Li, X.; Hratchian, H. P.; Izmaylov, A. F.; Bloino, J.; Zheng, G.; Sonnenberg, J. L.; Hada, M.; Ehara, M.; Toyota, K.; Fukuda, R.; Hasegawa, J.; Ishida, M.; Nakajima, T.; Honda, Y.; Kitao, O.; Nakai, H.; Vreven, T.; Montgomery Jr., J. A. J.; Peralta, E.; Ogliaro, F.; Bearpark, M.; Heyd, J. J.; Brothers, E.; Kudin, K. N.; Staroverov, V. N.; Keith, T.; Kobayashi, R.; Normand, J.; Raghavachari, K.; Rendell, A.; Burant, J. C.; Iyengar, S. S.; Tomasi, J.; Cossi, M.; Rega, N.; Millam, J. M.; Klene, M.; Knox, J. E.; Cross, J. B.; Bakken, V.; Adamo, C.; Jaramillo, J.; Gomperts, R.; Stratmann, R. E.; Yazyev, O.; Austin, A. J.; Cammi, R.; Pomelli, C.; Ochterski, J. W.; Martin, R. L.; Morokuma, K.; Zakrzewski, V. G.; Voth, G. A.; Salvador, P.; Dannenberg, J. J.; Dapprich, S.; Daniels, A. D.; Farkas, O.; Foresman, J. B.; Ortiz, J. V.; Cioslowski, J.; Fox, D. J. Gaussian, Inc., Wallingford CT, 2013. 


\section{${ }^{1} \mathrm{H},{ }^{13} \mathrm{C}$, and ${ }^{19} \mathrm{~F}$ NMR spectra of all new compounds}

Ethyl 3-(cyclohexylamino)-4,4,4-trifluoro-2-nitrobutanoate<smiles>CCOC(=O)C(=C(NC1CCCCC1)C(F)(F)F)[N+](=O)[O-]</smiles>

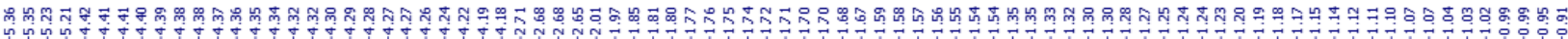
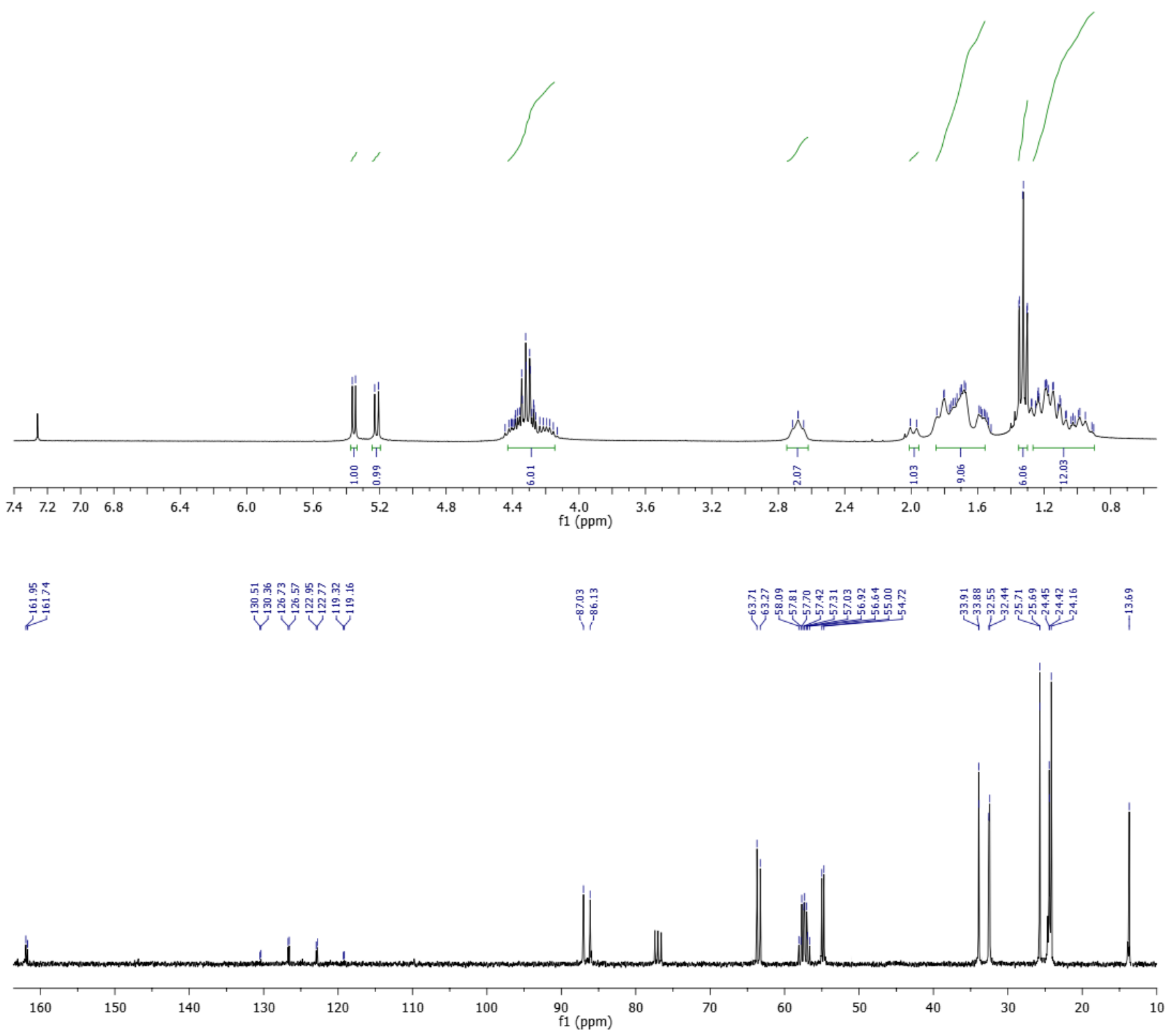


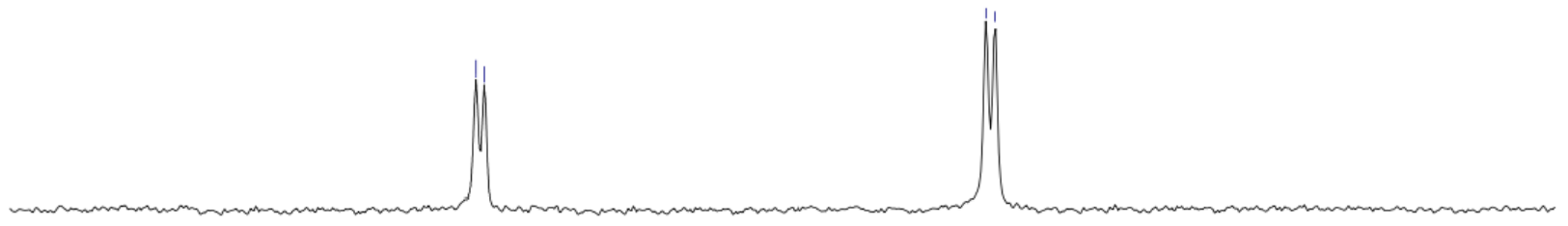

$\begin{array}{llllllllllllllllllllllllll}-69.6 & -69.8 & -70.0 & -70.2 & -70.4 & -70.6 & -70.8 & -71.0 & -71.2 & -71.4 & -71.6 & -71.8 & -72.0 & -72.2 & -72.4 & -72.6 & -72.8 & -73.0 & -73.2 & -73.4 & -73.6 & -73.8 & -74.0\end{array}$ 
Ethyl 3-(benzylamino)-4,4,4-trifluoro-2-nitrobutanoate<smiles>CCOC(=O)C(NCc1ccccc1)=C([N+](=O)[O-])C(F)(F)F</smiles>
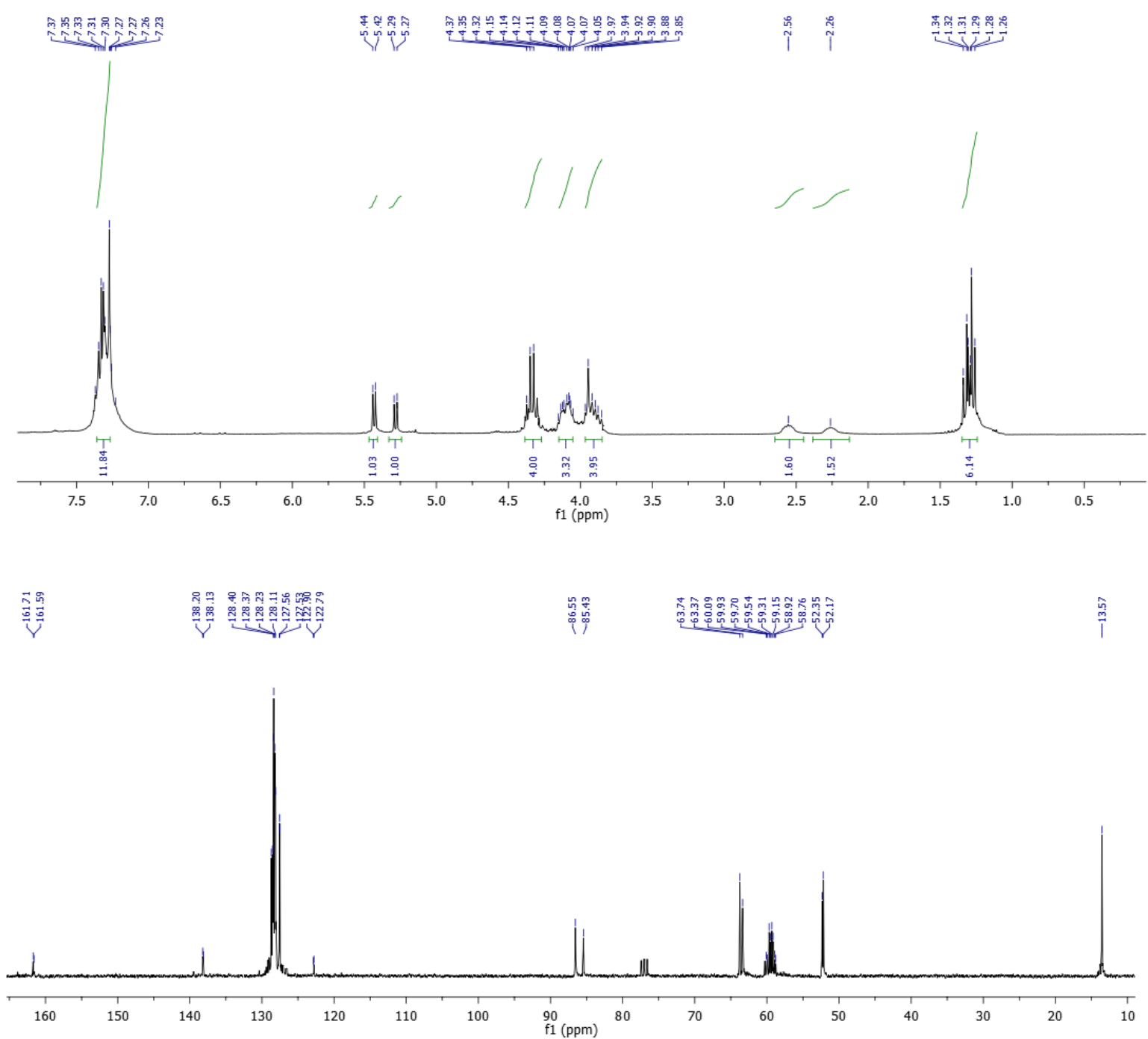


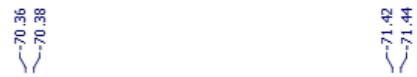

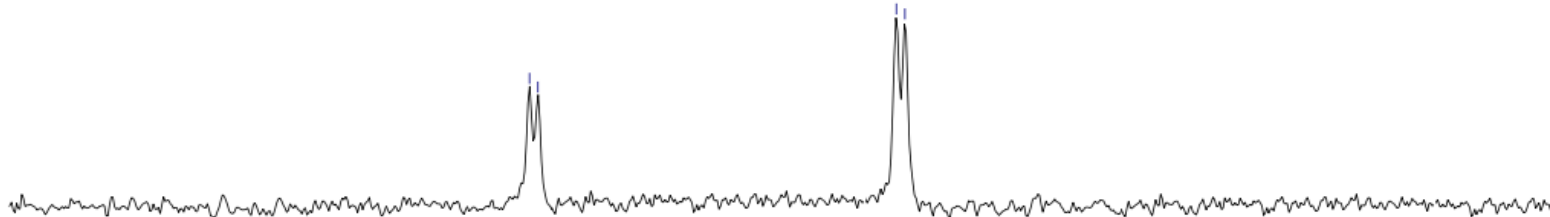

$\begin{array}{llllllllllllllllllllllll}-69.0 & -69.2 & -69.4 & -69.6 & -69.8 & -70.0 & -70.2 & -70.4 & -70.6 & -70.8 & -71.0 & -71.2 & -71.4 & -71.6 & -71.8 & -72.0 & -72.2 & -72.4 & -72.6 & -72.8 & -73.0 & -73.2\end{array}$ 
Ethyl 3-(cyclopentylamino)-4,4,4-trifluoro-2-nitrobutanoate<smiles>CCOC(=O)C(=C(NC1CCCC1)C(F)(F)F)[N+](=O)[O-]</smiles>

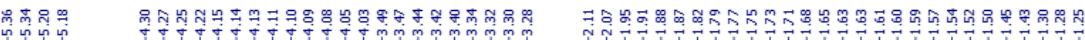
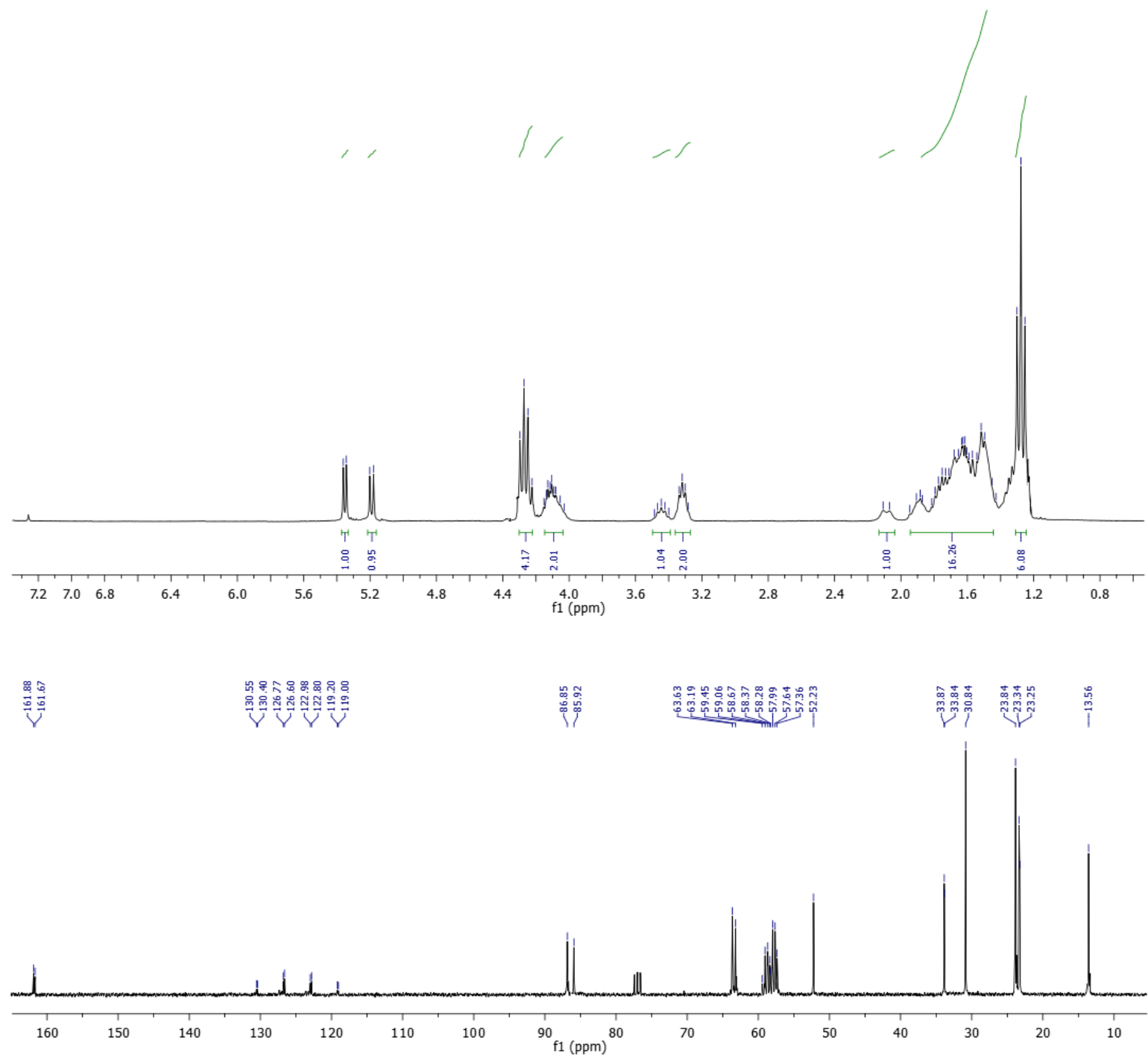


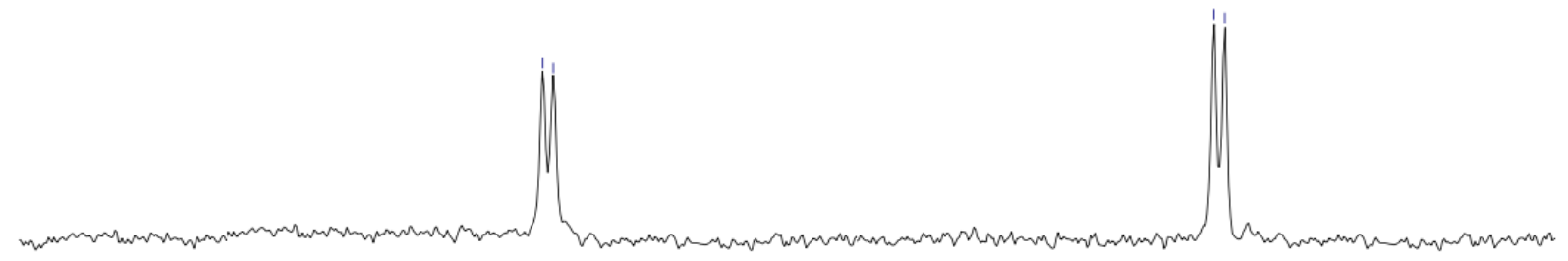

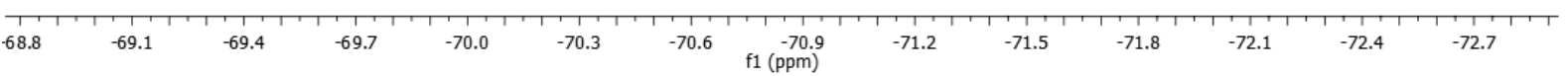


Ethyl 4,4,4-trifluoro-2-nitro-3-(pentylamino)butanoate<smiles>CCCCCNC(=C(COCC)C(F)(F)F)[N+](=O)[O-]</smiles>

3,3'd

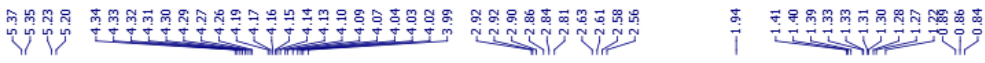
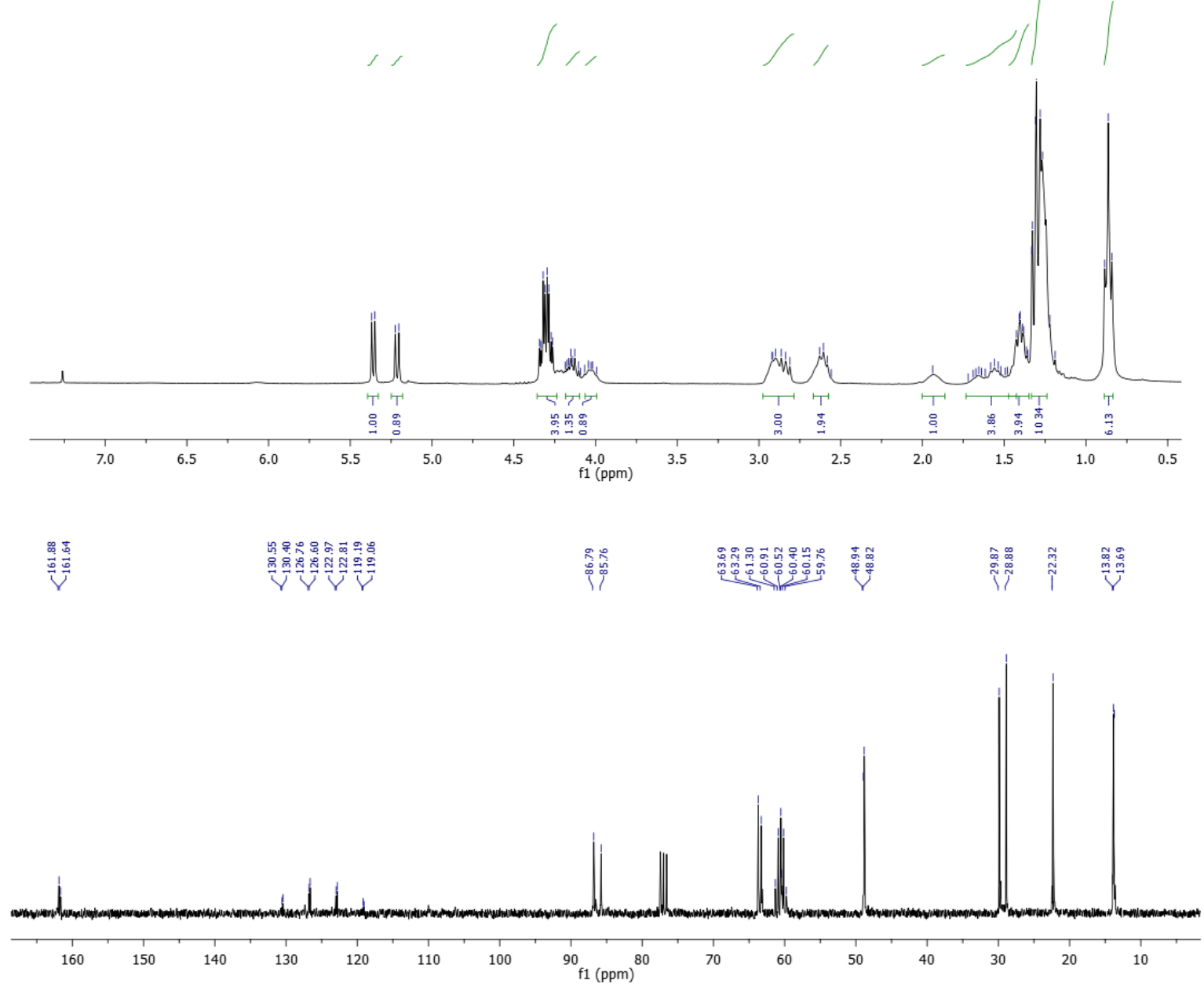
语

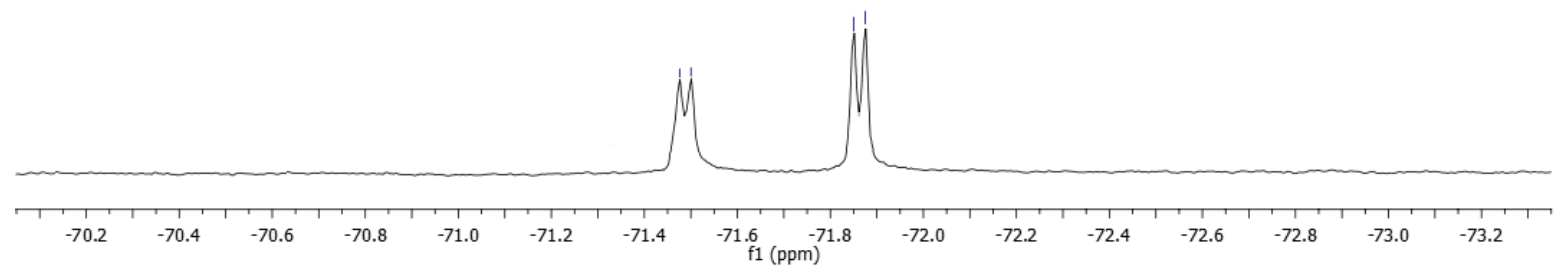


Ethyl 4,4,4-trifluoro-3-[(3-methoxy-3-oxopropyl)amino]-2-nitrobutanoate<smiles>CCOC(=O)C(NCCC(=O)OC)=C([N+](=O)[O-])C(F)(F)F</smiles>

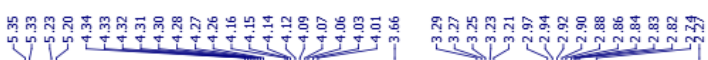
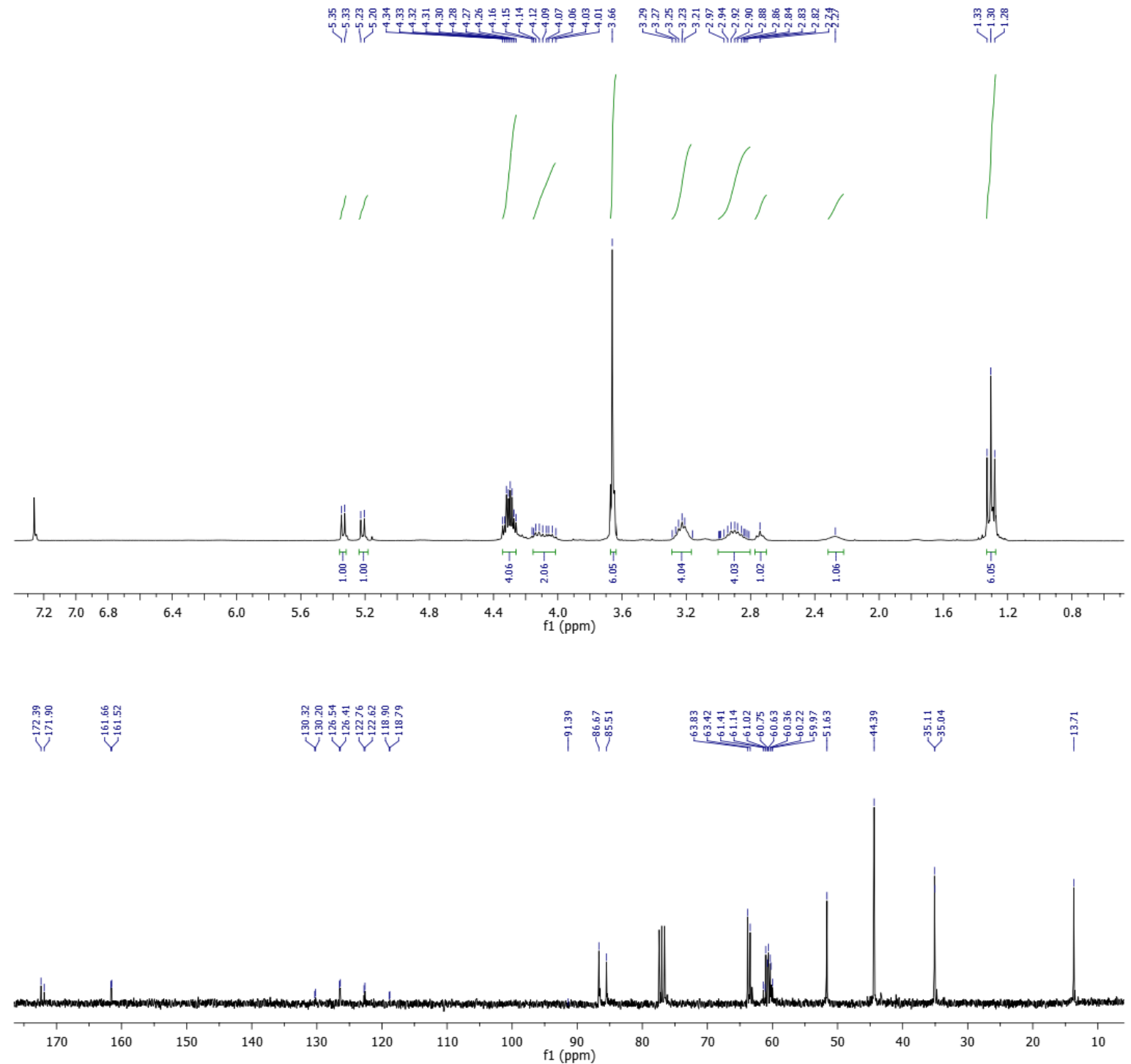


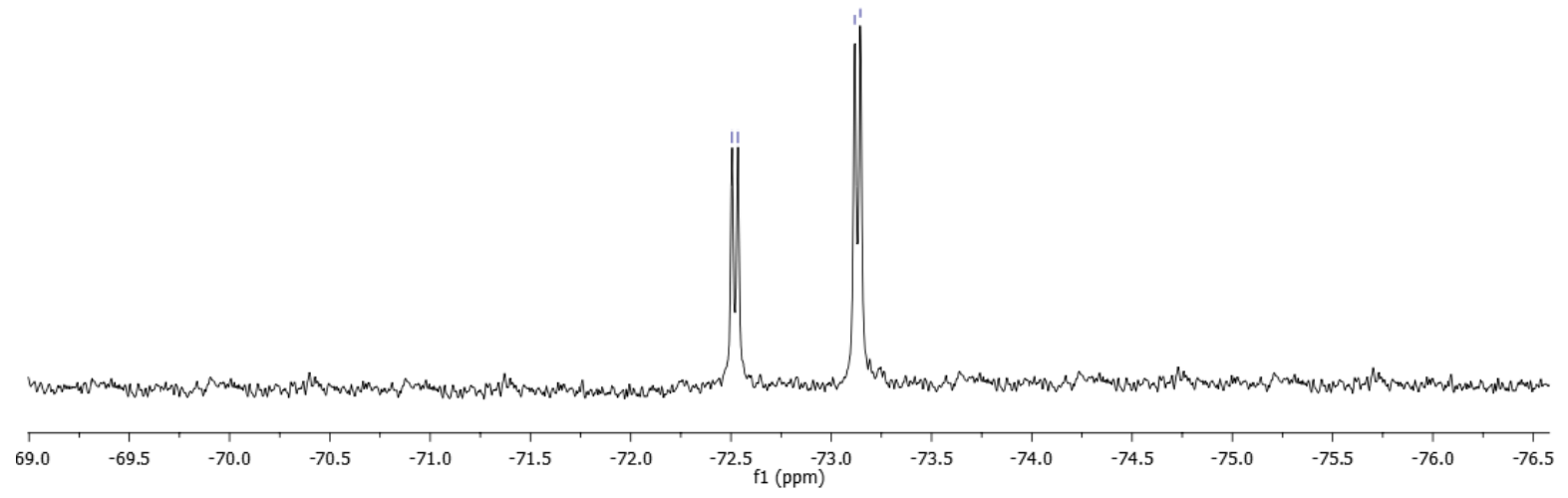


Ethyl 4,4,4-trifluoro-3-[(4-methoxyphenyl)amino]-2-nitrobutanoate<smiles>CCOC(=O)C(Nc1ccc(OC)cc1)=C([N+](=O)[O-])C(F)(F)F</smiles>

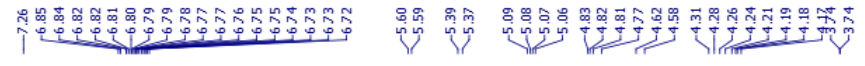
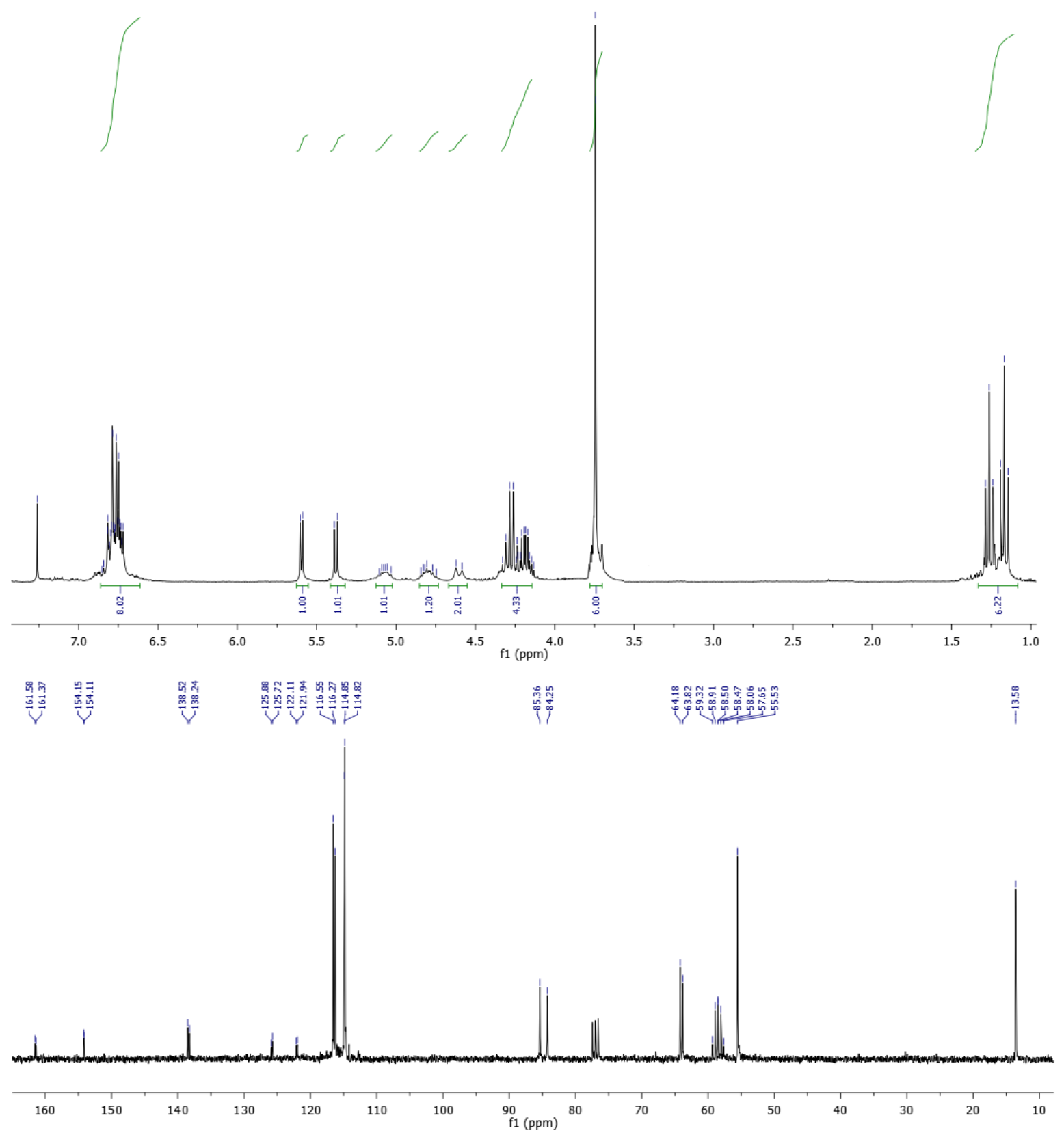


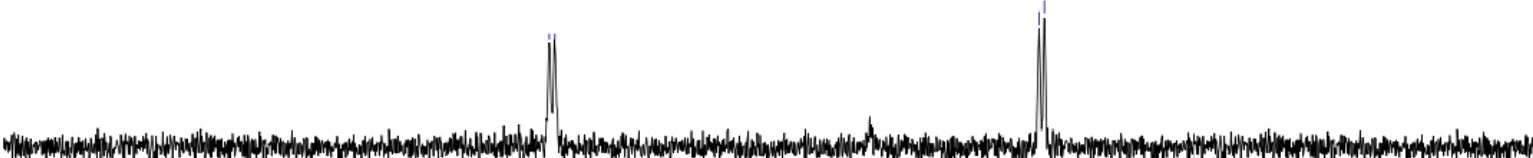
M

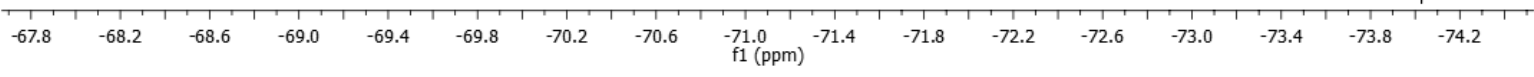


Ethyl 2-amino-3-(cyclohexylamino)-4,4,4-trifluorobutanoate<smiles>CCOC(N)[C@H](NC1CCCCC1)C(F)(F)F</smiles><smiles>CCOC(N)[C@H](NC1CCCCC1)C(F)(F)F</smiles>

syn-4a

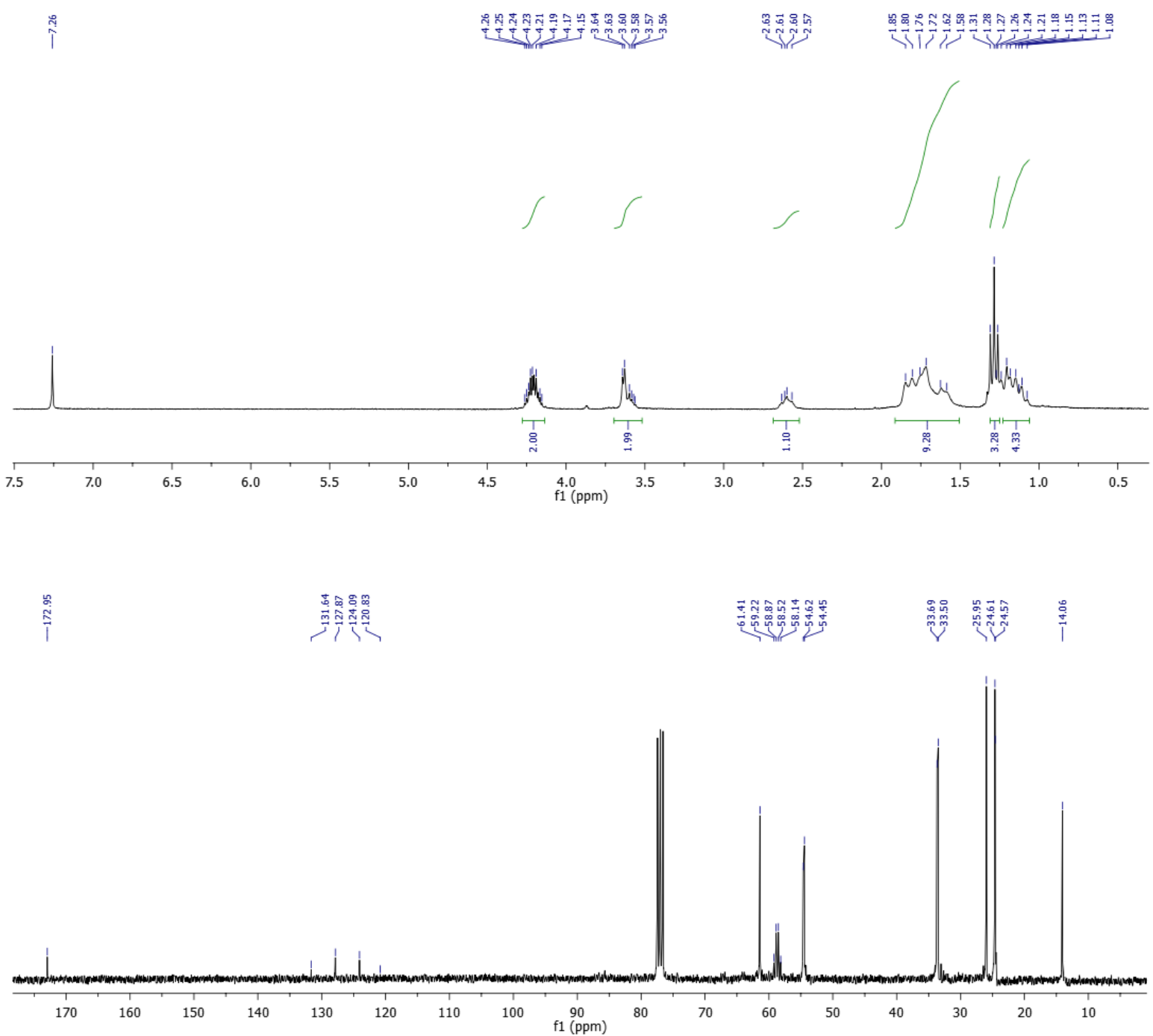




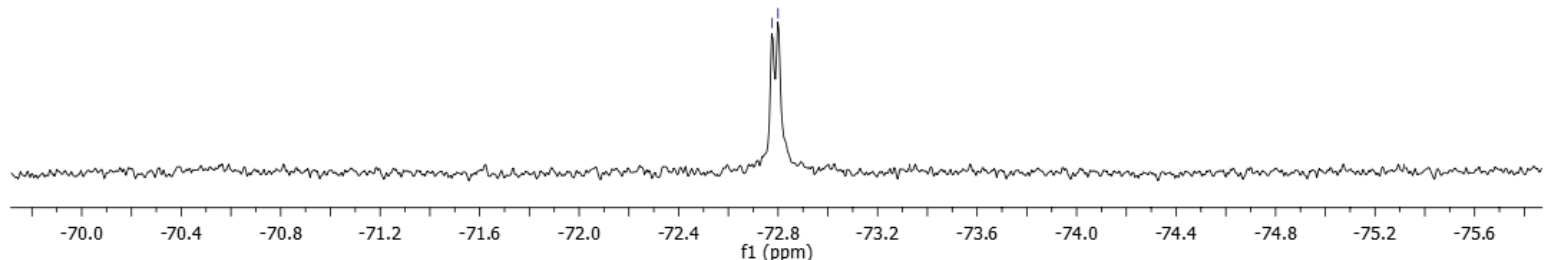


Ethyl 2-amino-3-(cyclohexylamino)-4,4,4-trifluorobutanoate<smiles>CCOC(N)[C@H](NC1CCCCC1)C(F)(F)F</smiles><smiles>CCOC(N)[C@H](NC1CCCCC1)C(F)(F)F</smiles>

anti-4'a

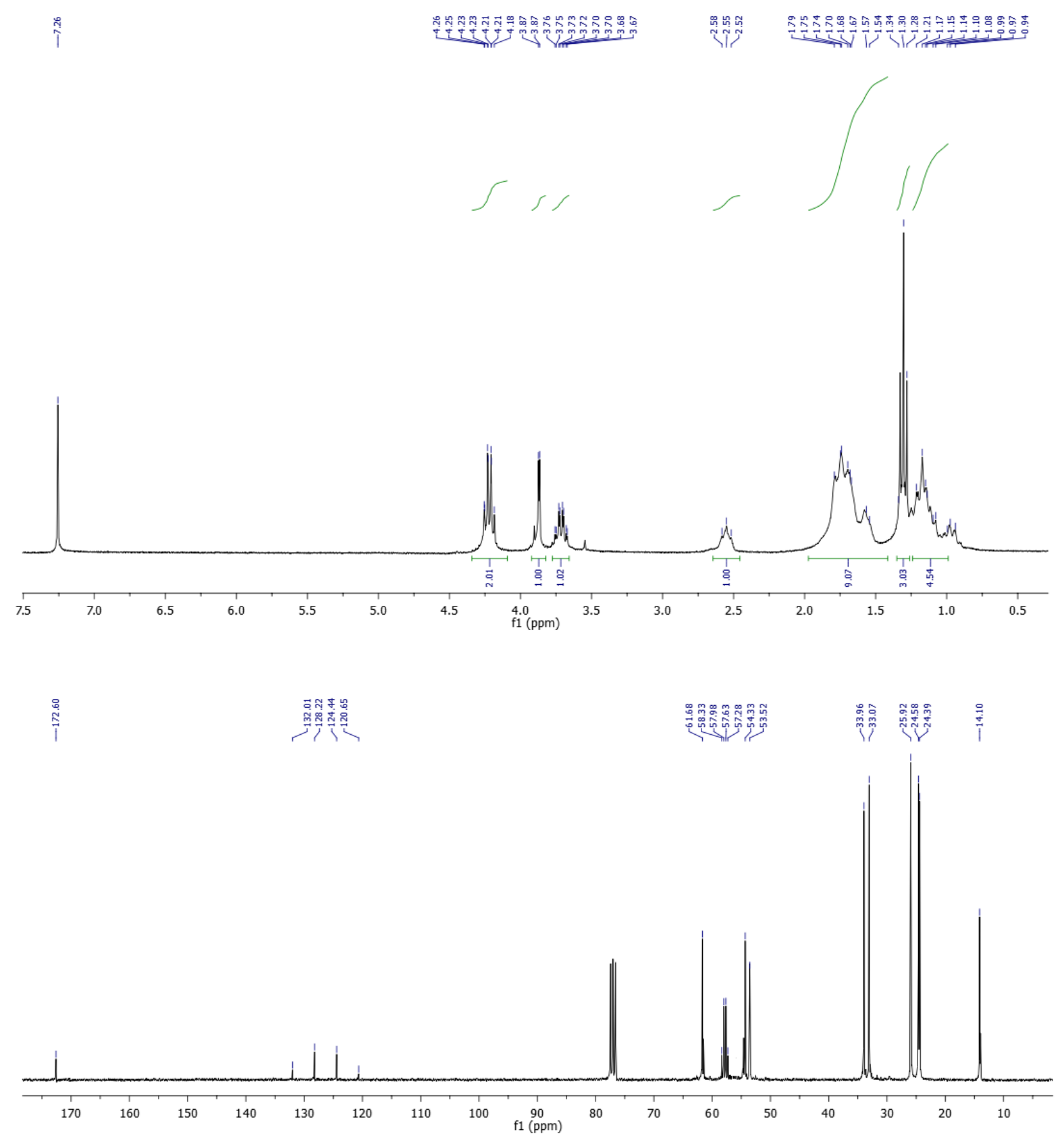




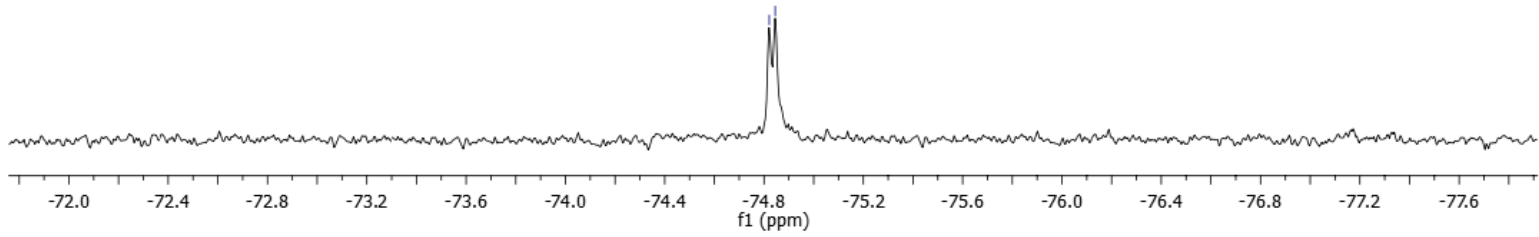




\section{Ethyl 2-amino-3-(benzylamino)-4,4,4-trifluorobutanoate}<smiles>CCOC(N)[C@H](NCc1ccccc1)C(F)(F)F</smiles><smiles>CCOC(N)[C@H](NCc1ccccc1)C(F)(F)F</smiles>

syn-4b

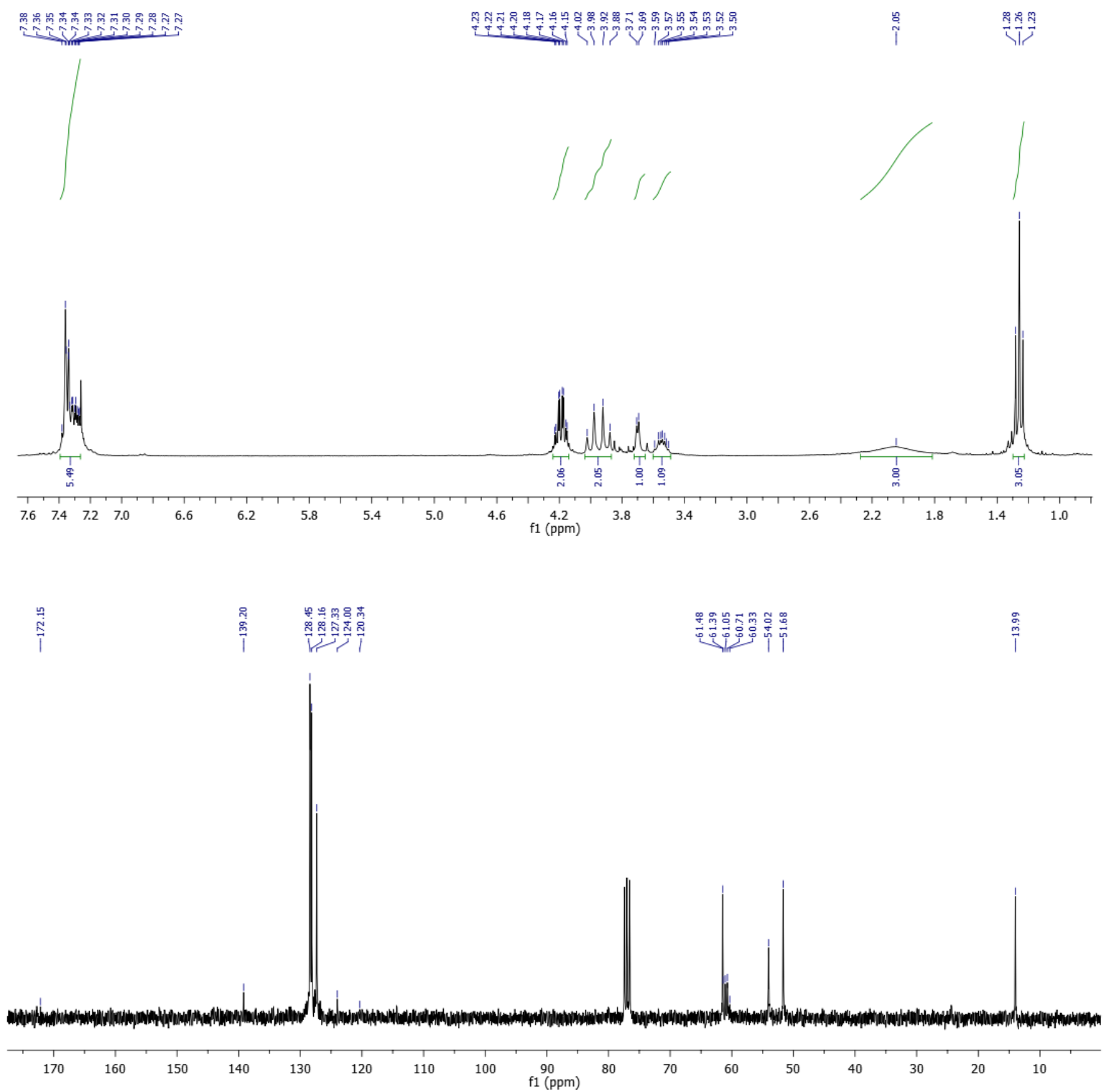




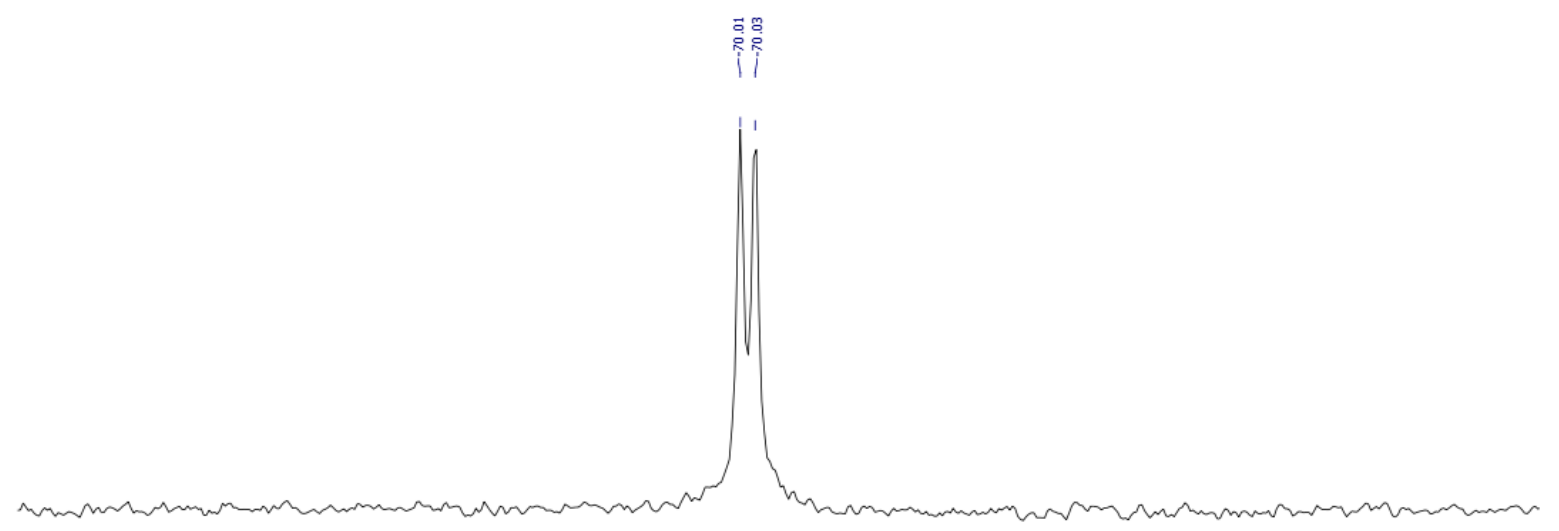

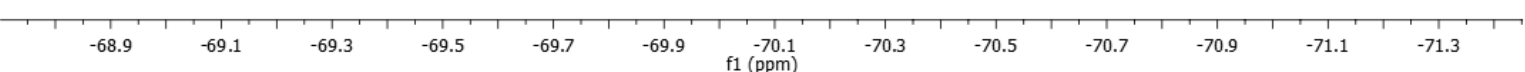


Ethyl 2-amino-3-(benzylamino)-4,4,4-trifluorobutanoate<smiles>CCOC(N)[C@H](NCc1ccccc1)C(F)(F)F</smiles><smiles>CCO[C@H](N)[C@H](NCc1ccccc1)C(F)(F)F</smiles>

anti-4'b

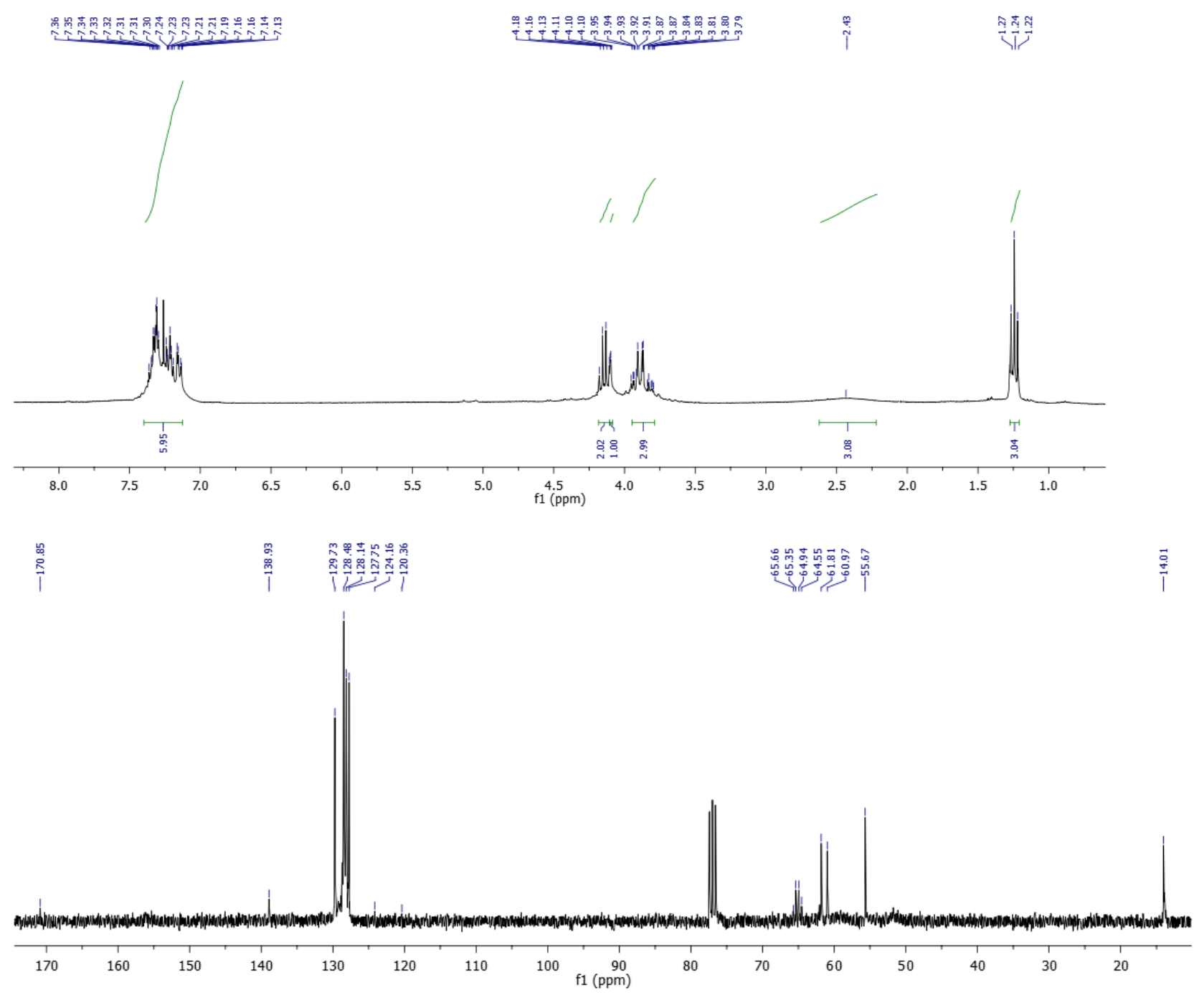




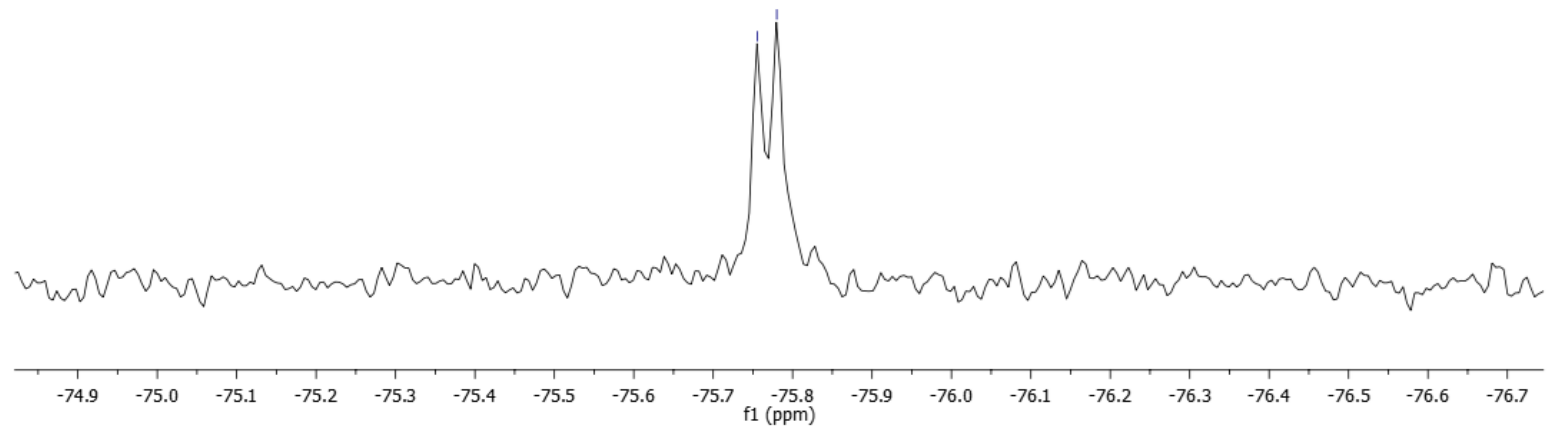


Ethyl 2-amino-4,4,4-trifluoro-3-[(4-methoxyphenyl)amino]butanoate<smiles>CCOC(N)[C@H](Nc1ccc(OC)cc1)C(F)(F)F</smiles><smiles>CCOC(N)[C@H](Nc1ccc(OC)cc1)C(F)(F)F</smiles>

syn-4f

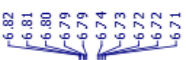

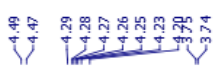

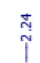
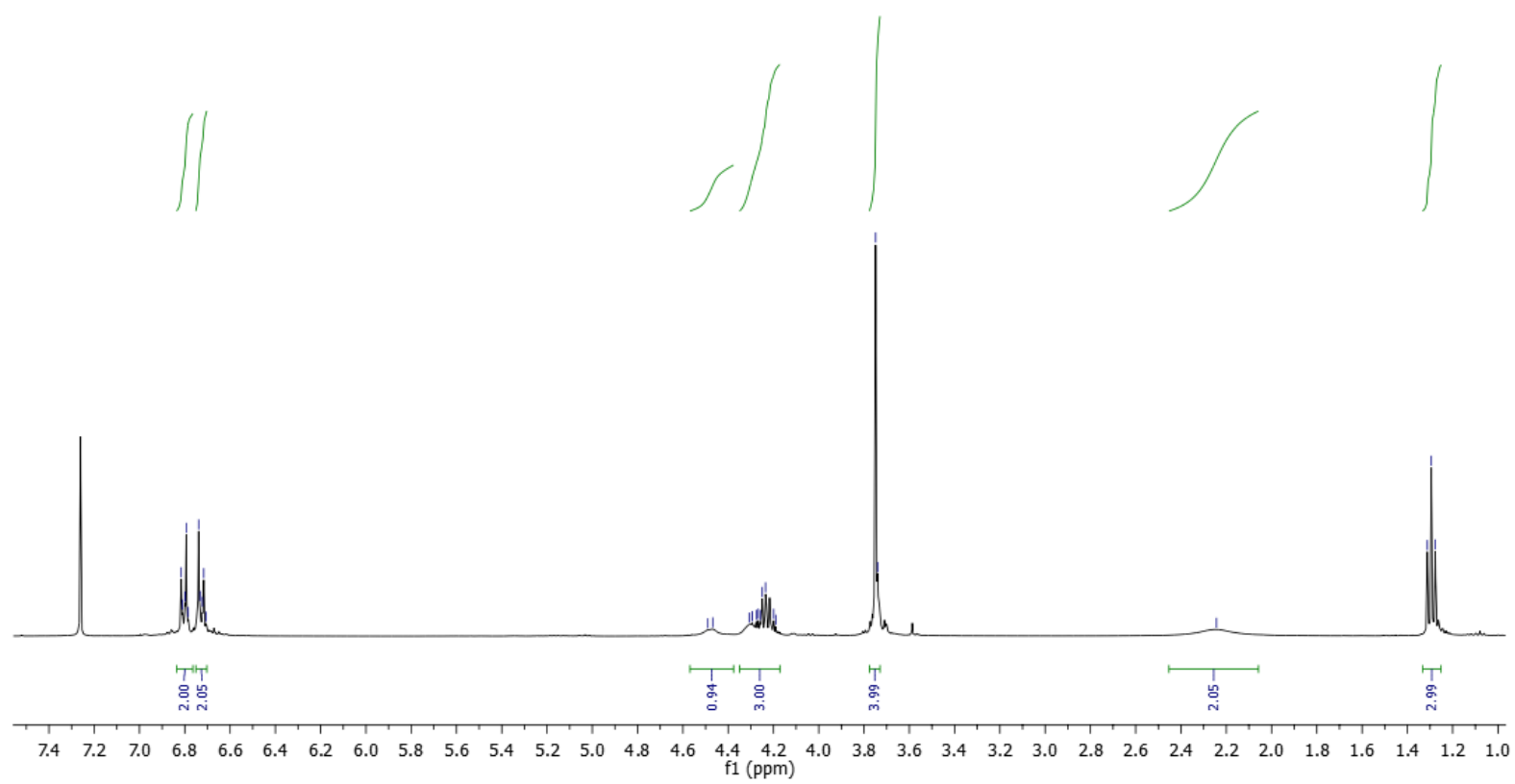

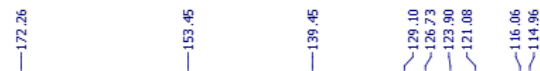

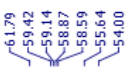

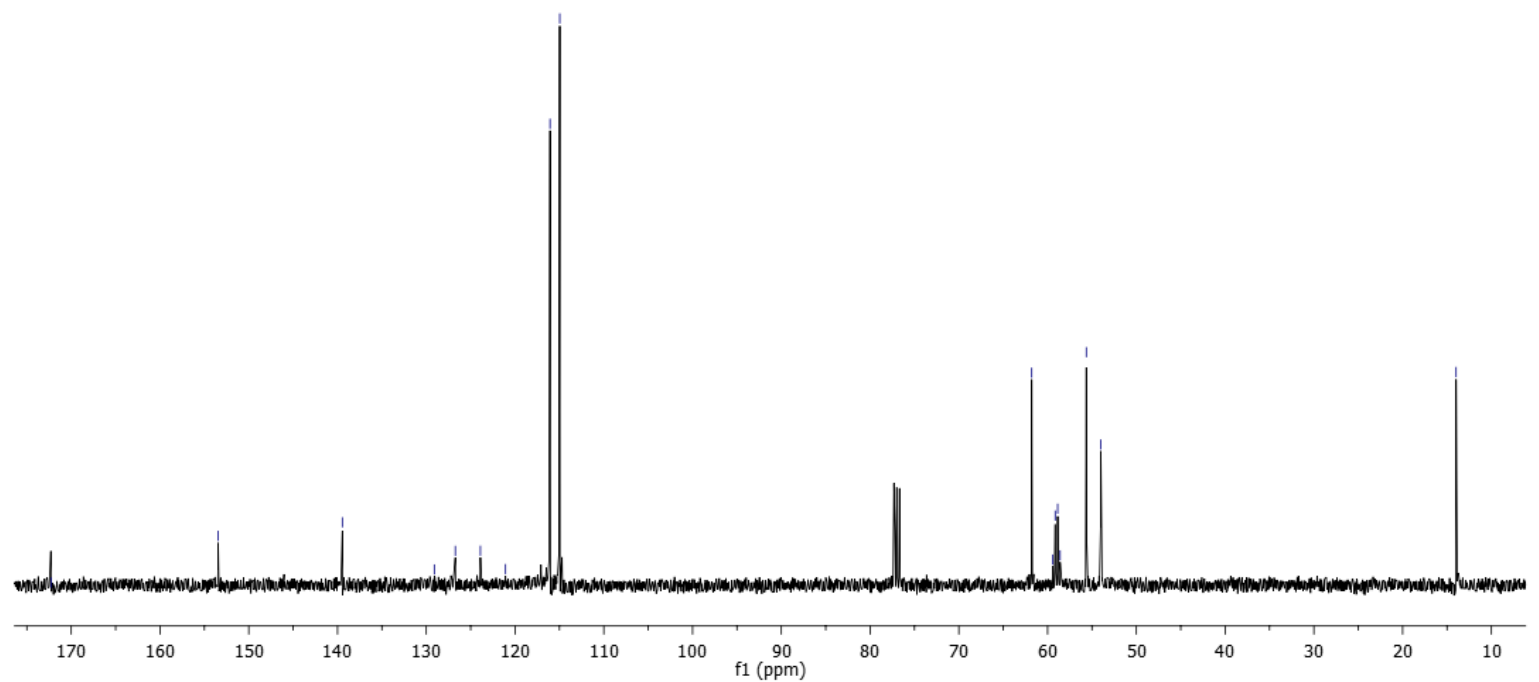




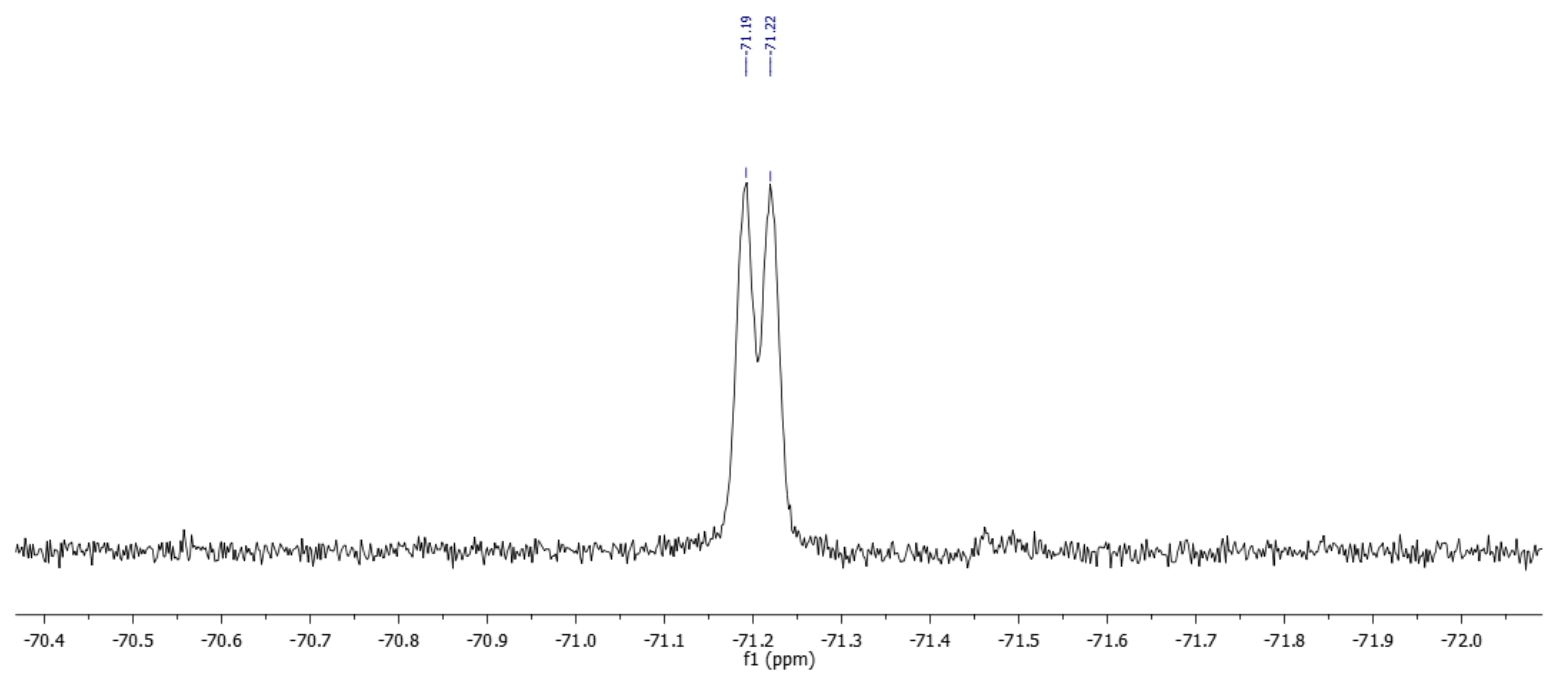


Ethyl 2-amino-4,4,4-trifluoro-3-[(4-methoxyphenyl)amino]butanoate<smiles>CCOC(N)[C@H](Nc1ccc(OC)cc1)C(F)(F)F</smiles><smiles>CCOC(N)[C@H](Nc1ccc(OC)cc1)C(F)(F)F</smiles>
anti-4'f

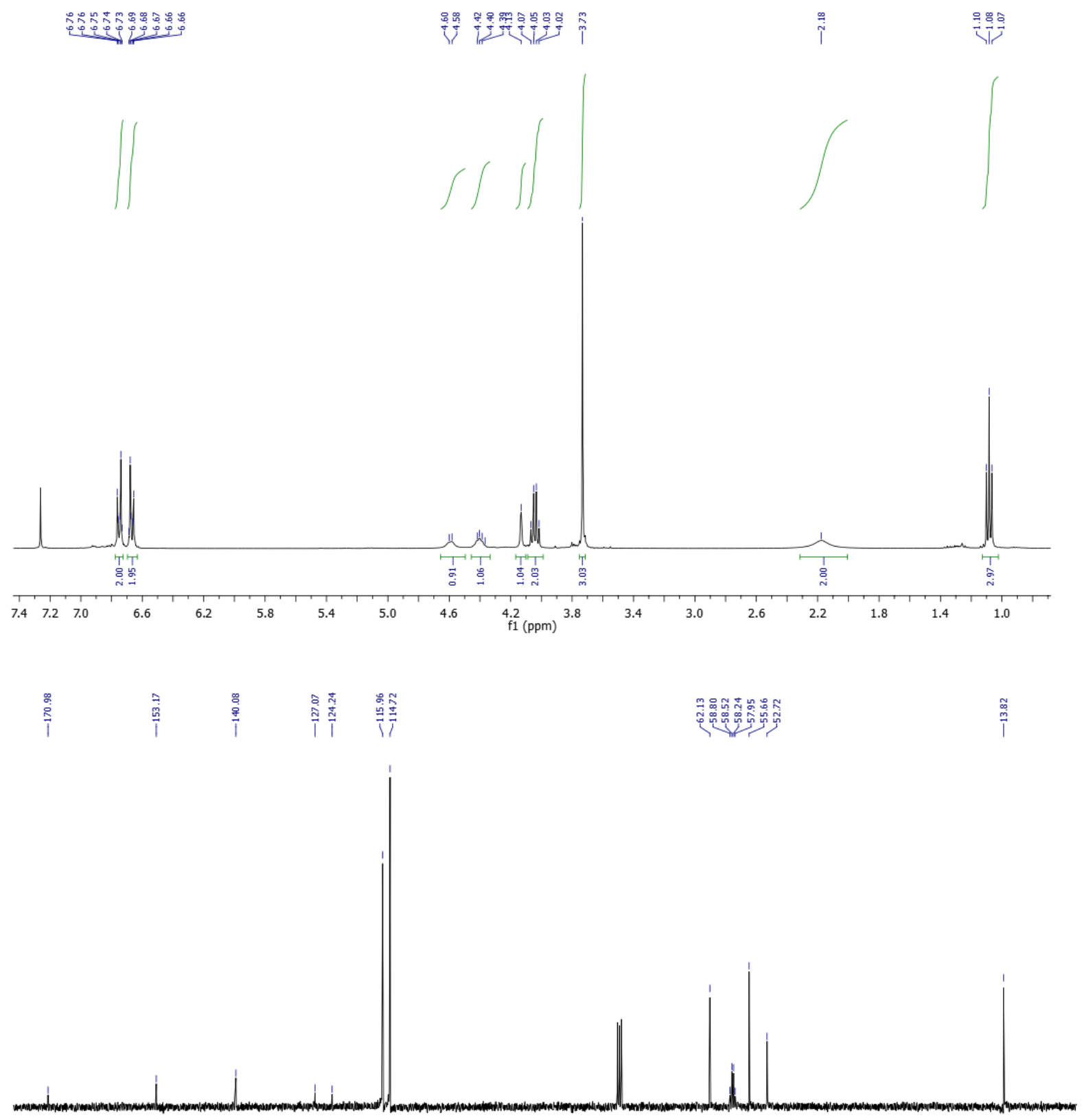

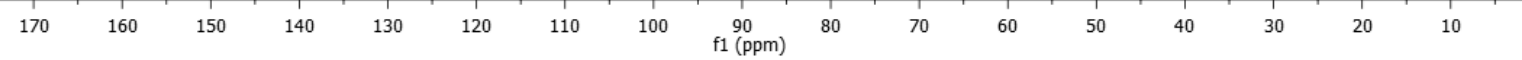




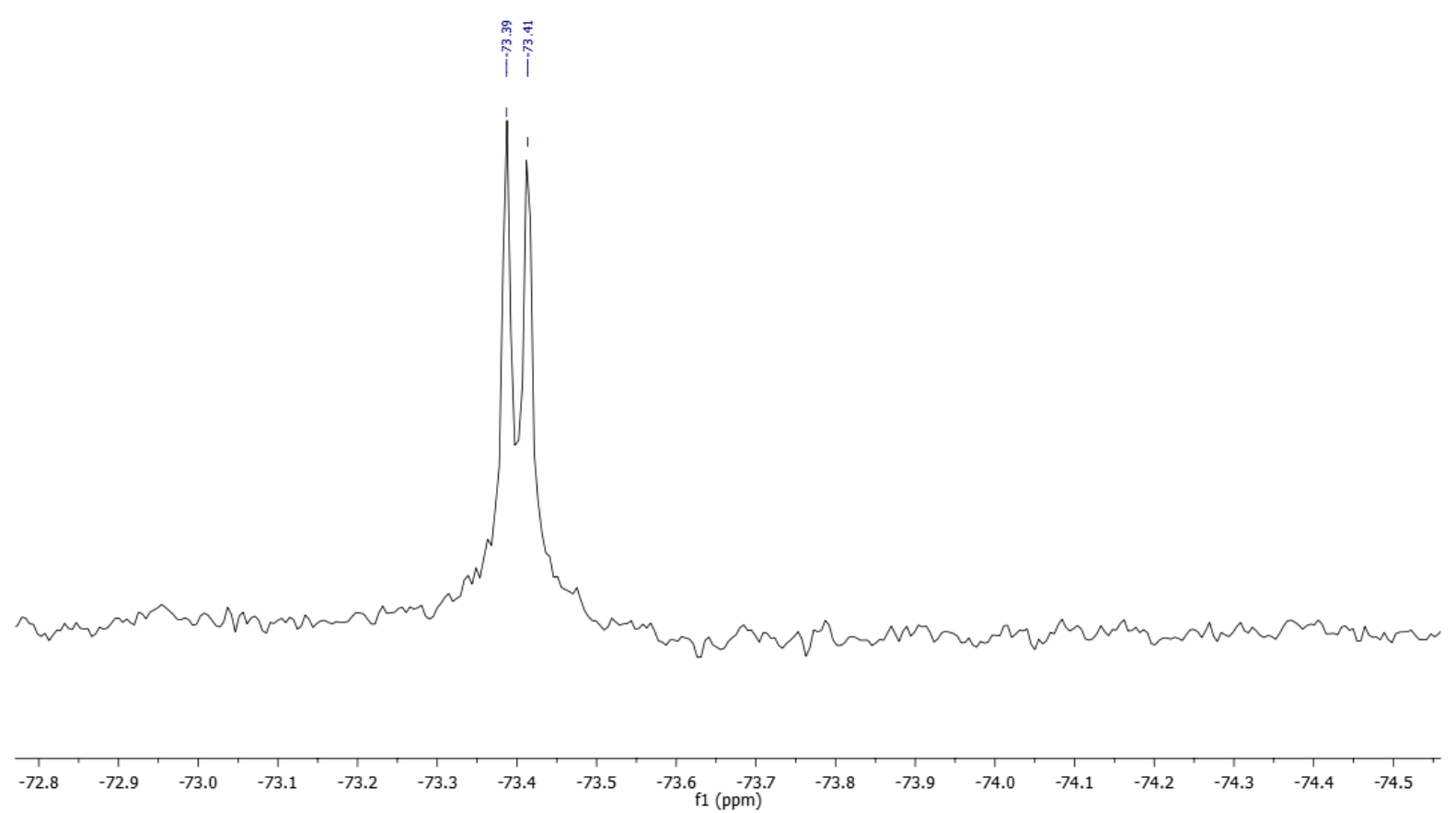


Ethyl 4,4,4-trifluoro-2-nitro-3-\{[(1R)-1-phenylethyl]amino\}butanoate<smiles>CCOC(=O)C(=C(C)N[C@H](C)c1ccccc1)C(F)(F)F</smiles>

syn-6,7g, anti-6',7'g
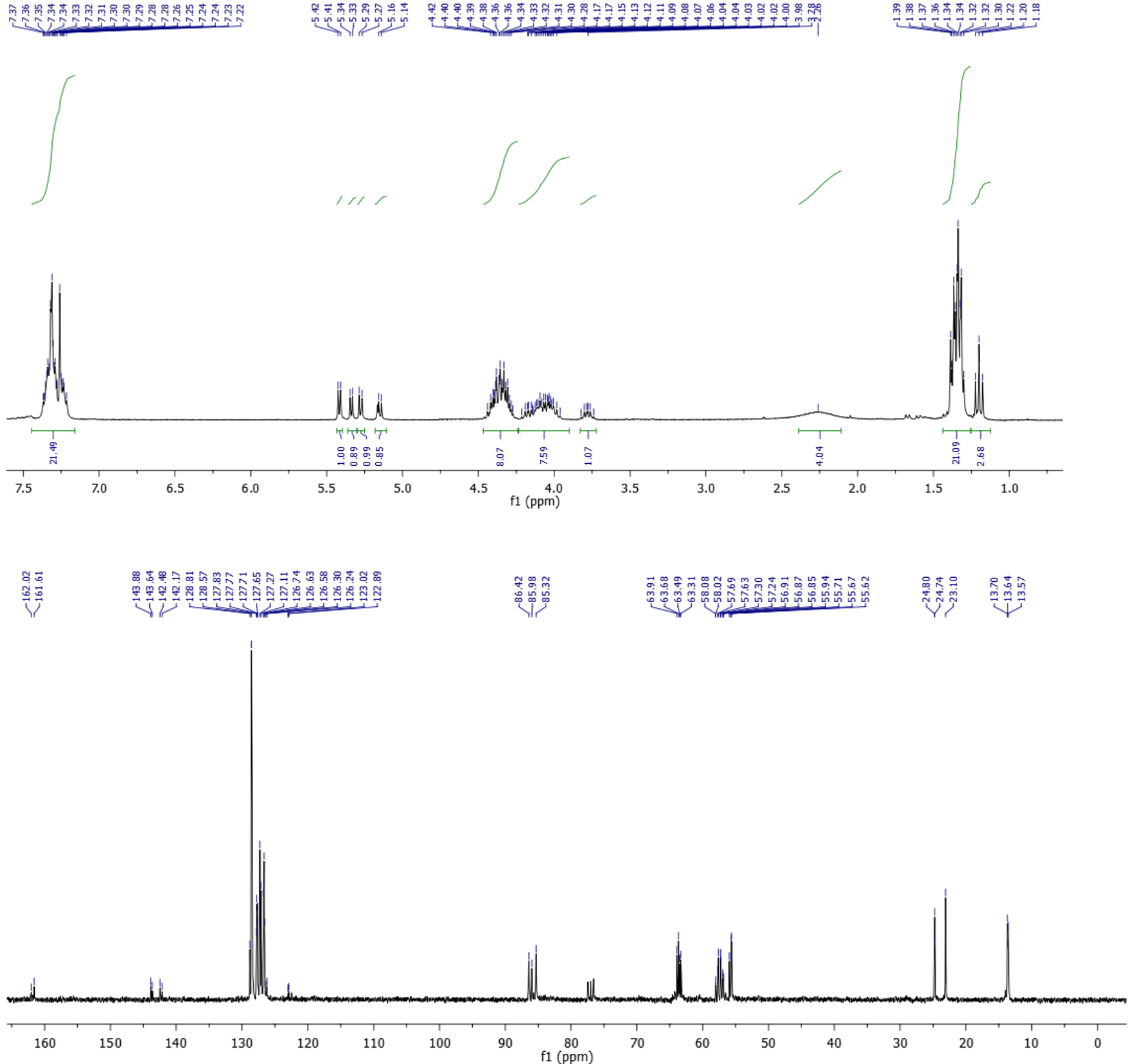


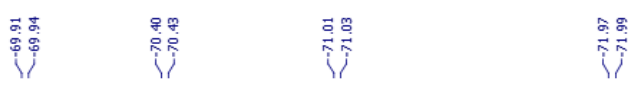

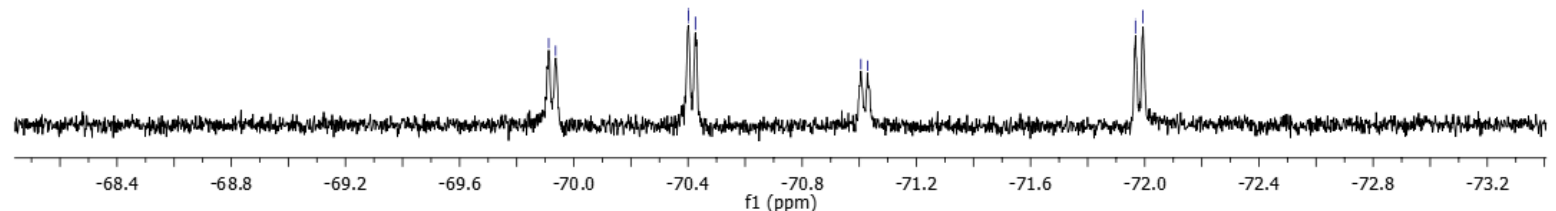


Ethyl (2S,3S)-2-amino-4,4,4-trifluoro-3-\{[(1R)-1-phenylethyl]amino\}butanoate<smiles>CCOC(=O)C(N)C(NC(C)c1ccccc1)C(F)(F)F</smiles>

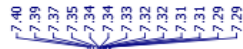

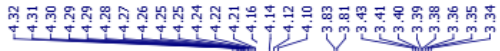

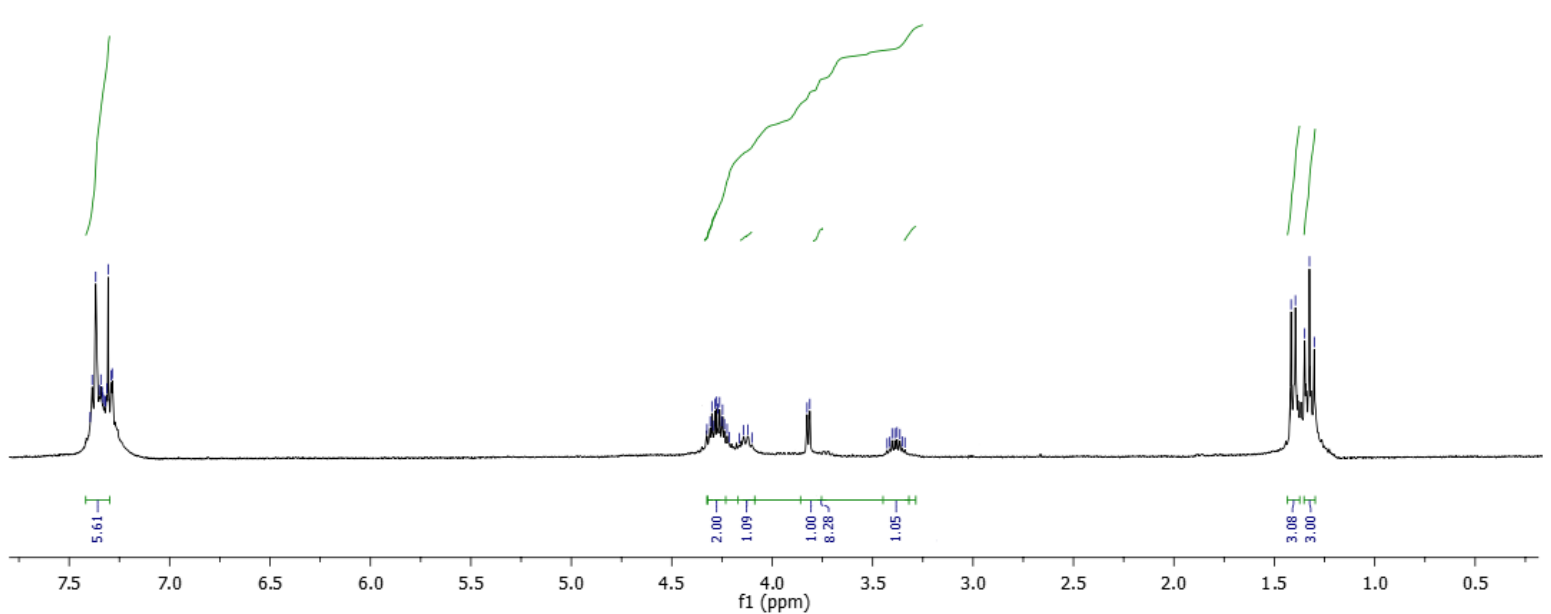

i⿱

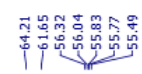

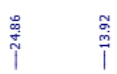

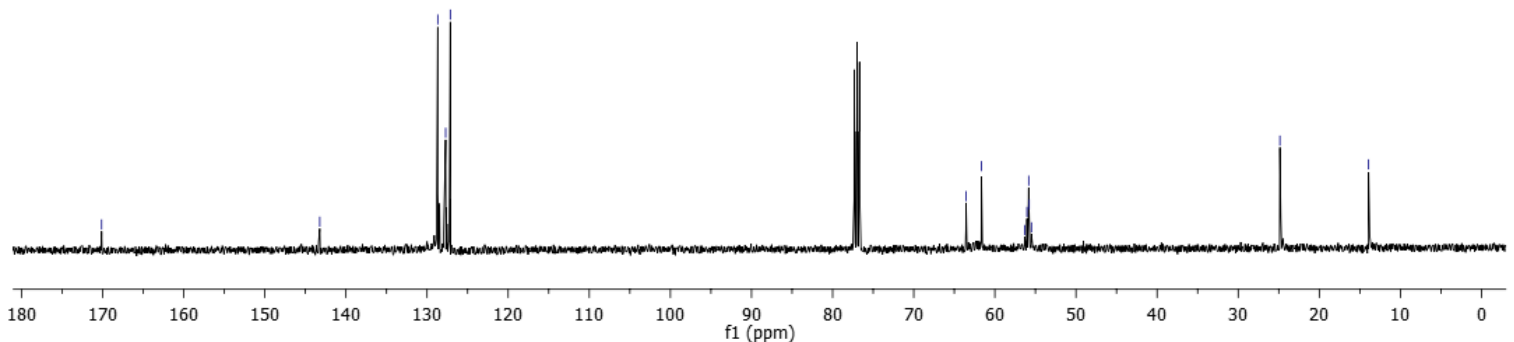




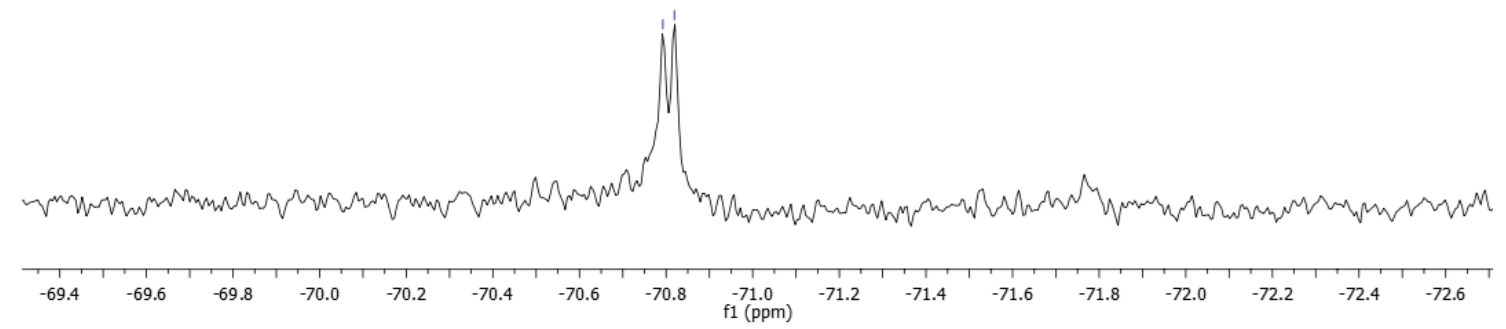


Ethyl (2R,3S)-2-amino-4,4,4-trifluoro-3-\{[(1R)-1-phenylethyl]amino\}butanoate<smiles>CCOC(=O)[C@H](N)[C@H](N[C@H](C)c1ccccc1)C(F)(F)F</smiles>

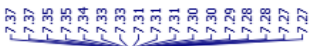

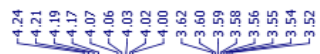
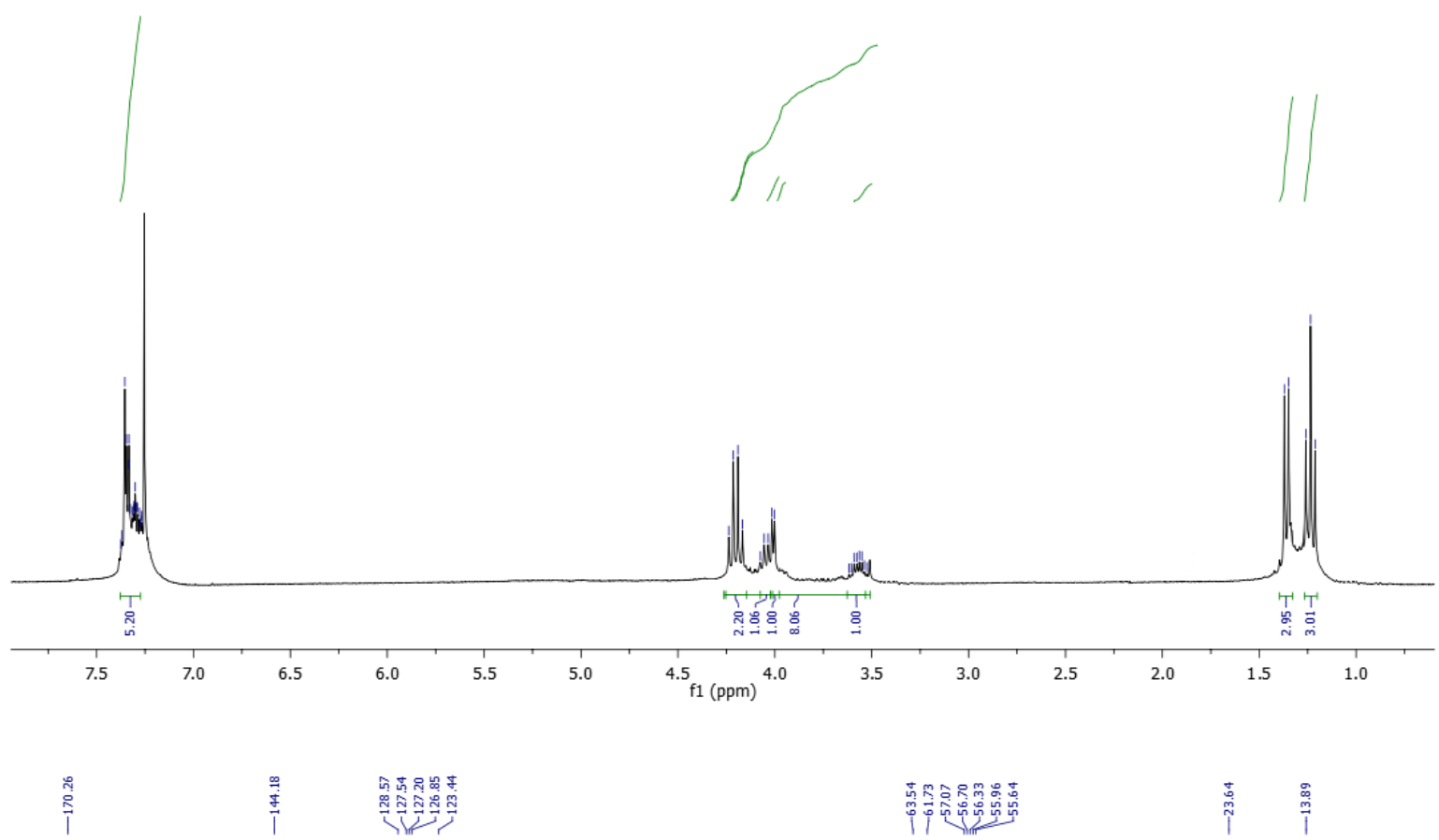

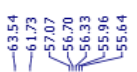

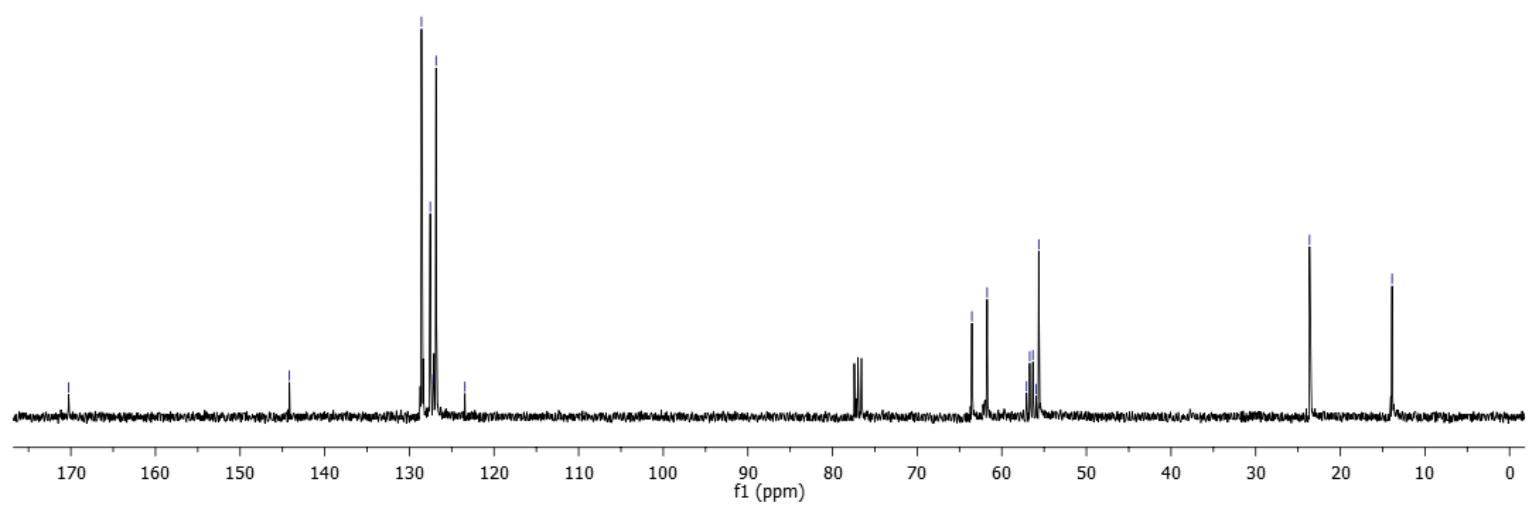


i

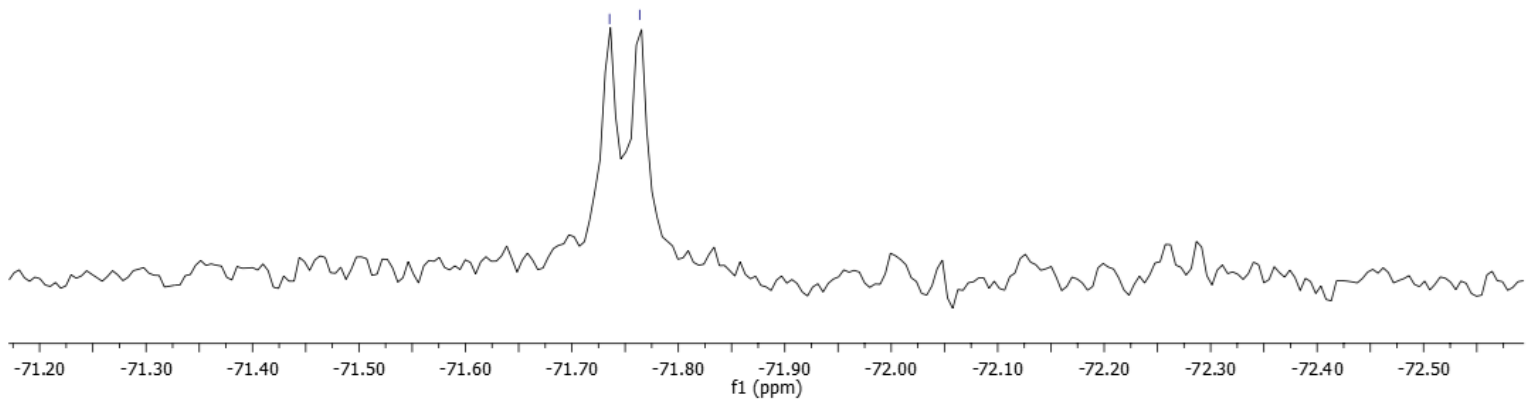


Ethyl (2R,3R)-2-amino-4,4,4-trifluoro-3-\{[(1R)-1-phenylethyl]amino $\}$ butanoate<smiles>CCOC(=O)[C@H](N)[C@@H](N[C@@H](C)c1ccccc1)C(F)(F)F</smiles>

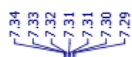

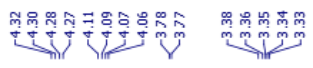

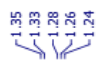
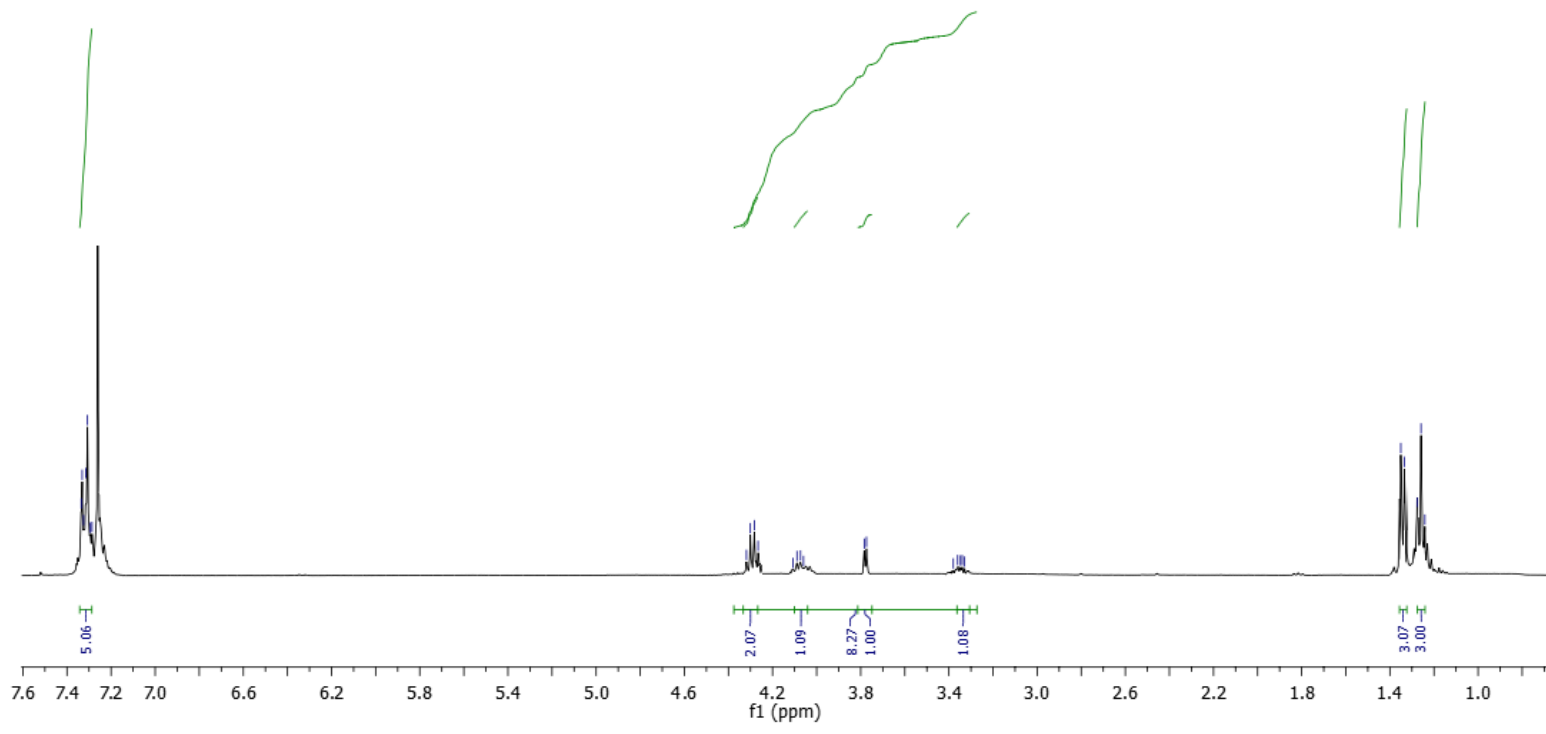

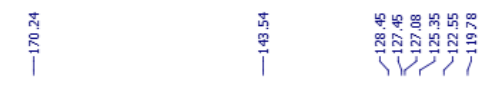

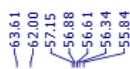

竓

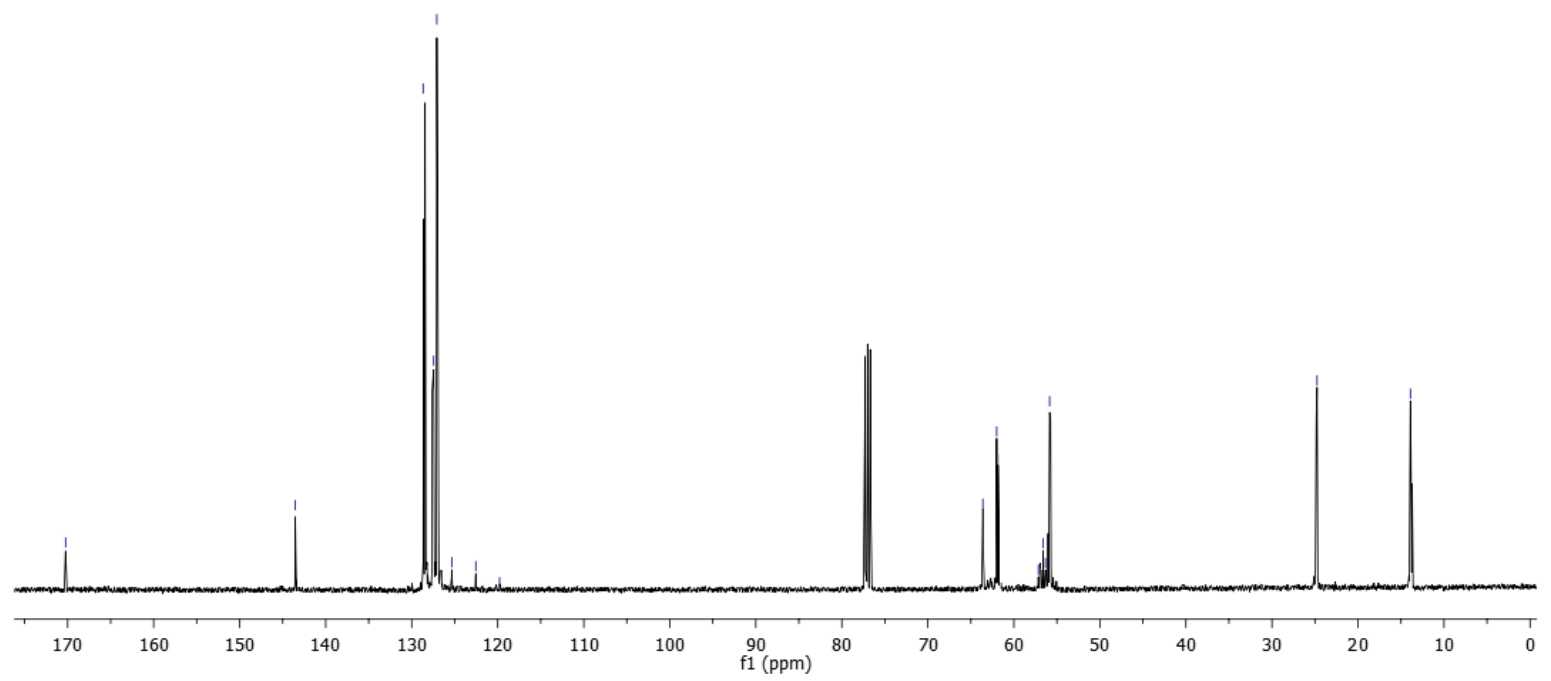

S33 
蓄

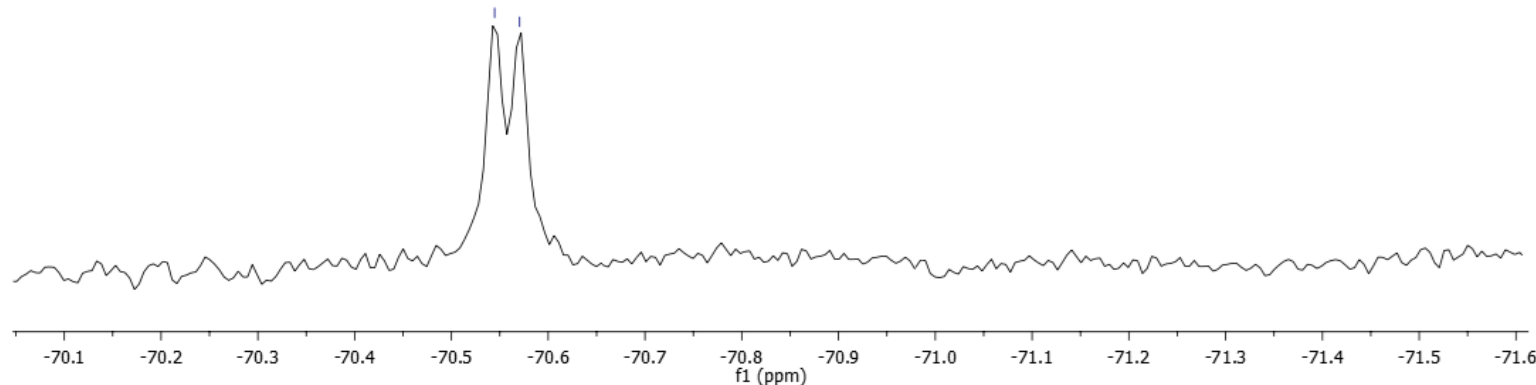


Ethyl (2S,3R)-2-amino-4,4,4-trifluoro-3-\{[(1R)-1-phenylethyl]amino\}butanoate<smiles>CCOC(=O)C(N)C(NC(C)c1ccccc1)C(F)(F)F</smiles>

anti-9'g

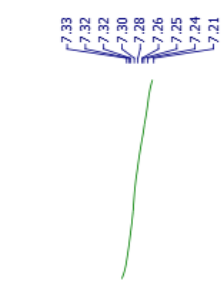

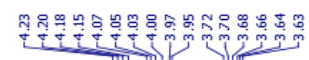

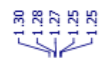

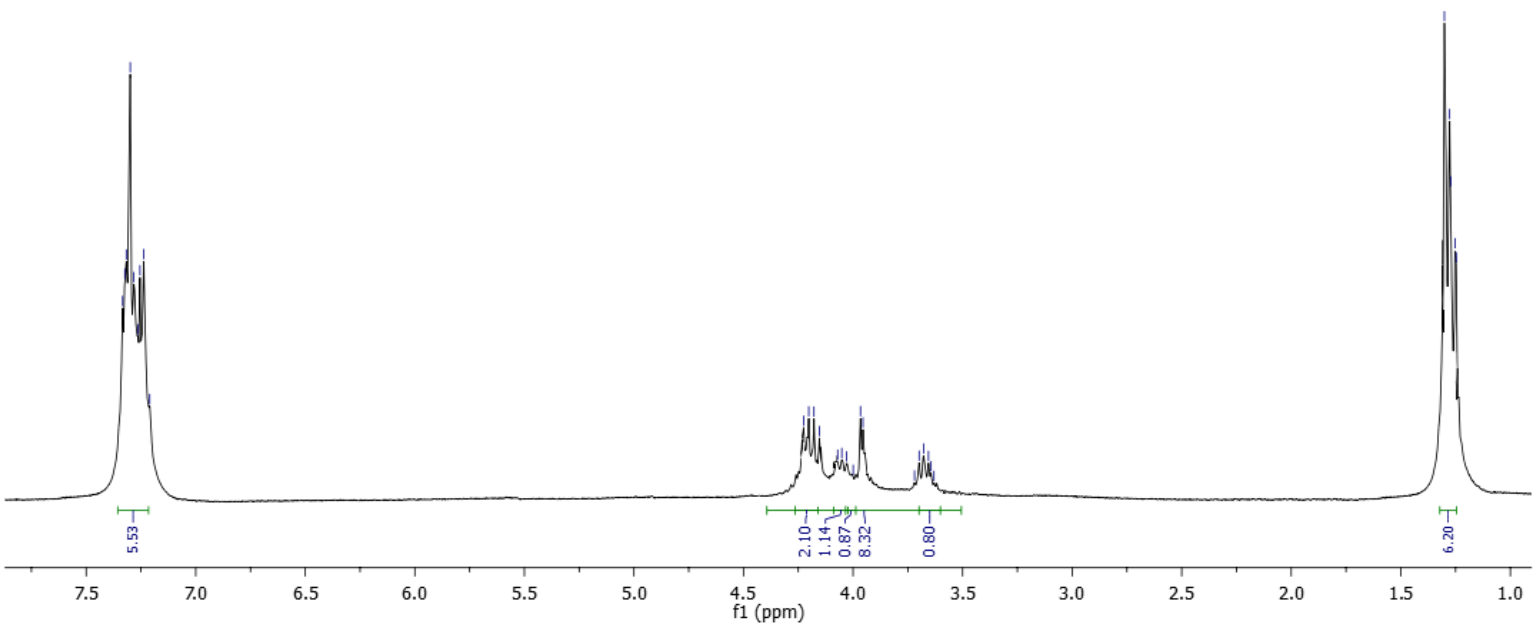

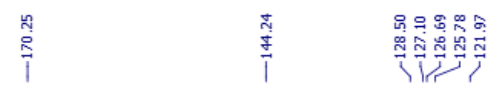

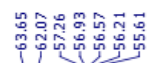

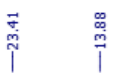

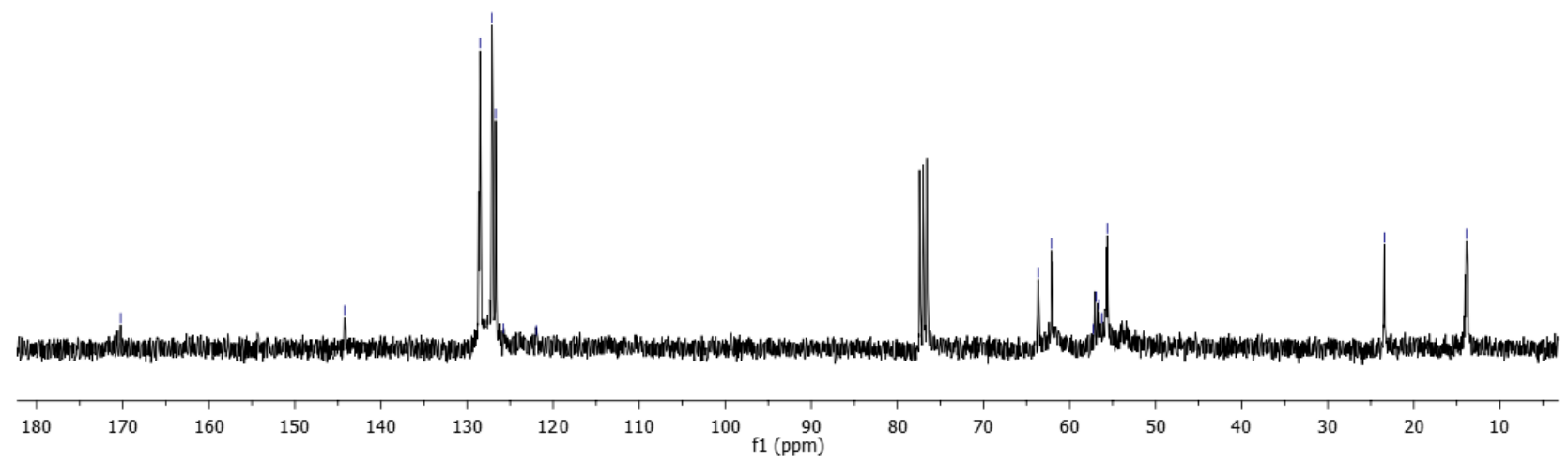




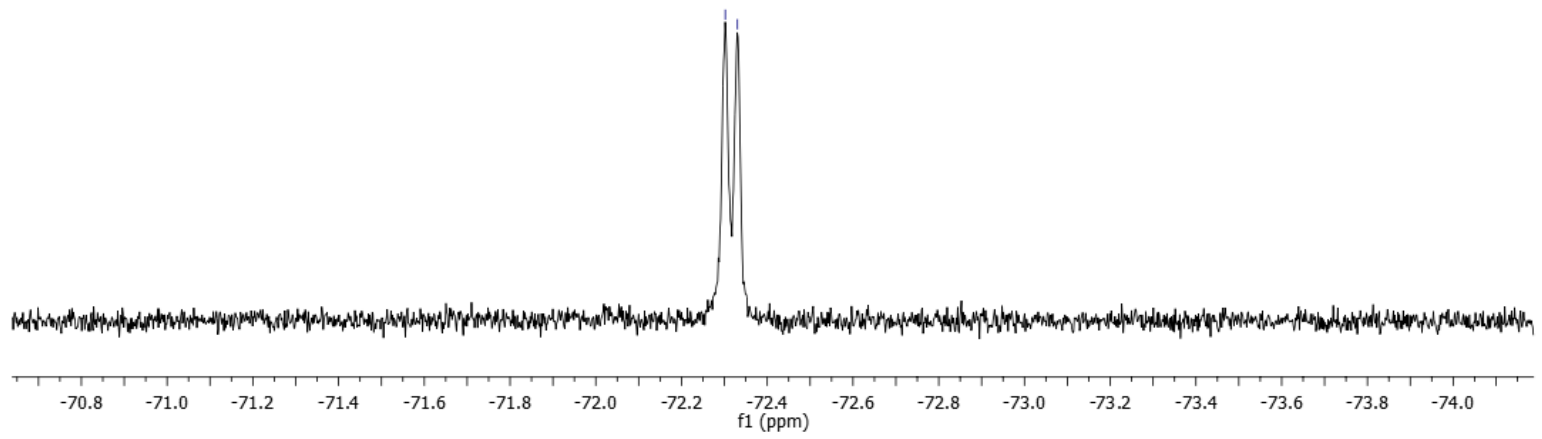


Methyl (2S,3R)-3-tert-butoxy-2-[(E)-(2,2,2-trifluoroethylidene)amino]butanoate<smiles>CCCCOC(C)C(N=CC(F)(F)F)C(C)OC</smiles>

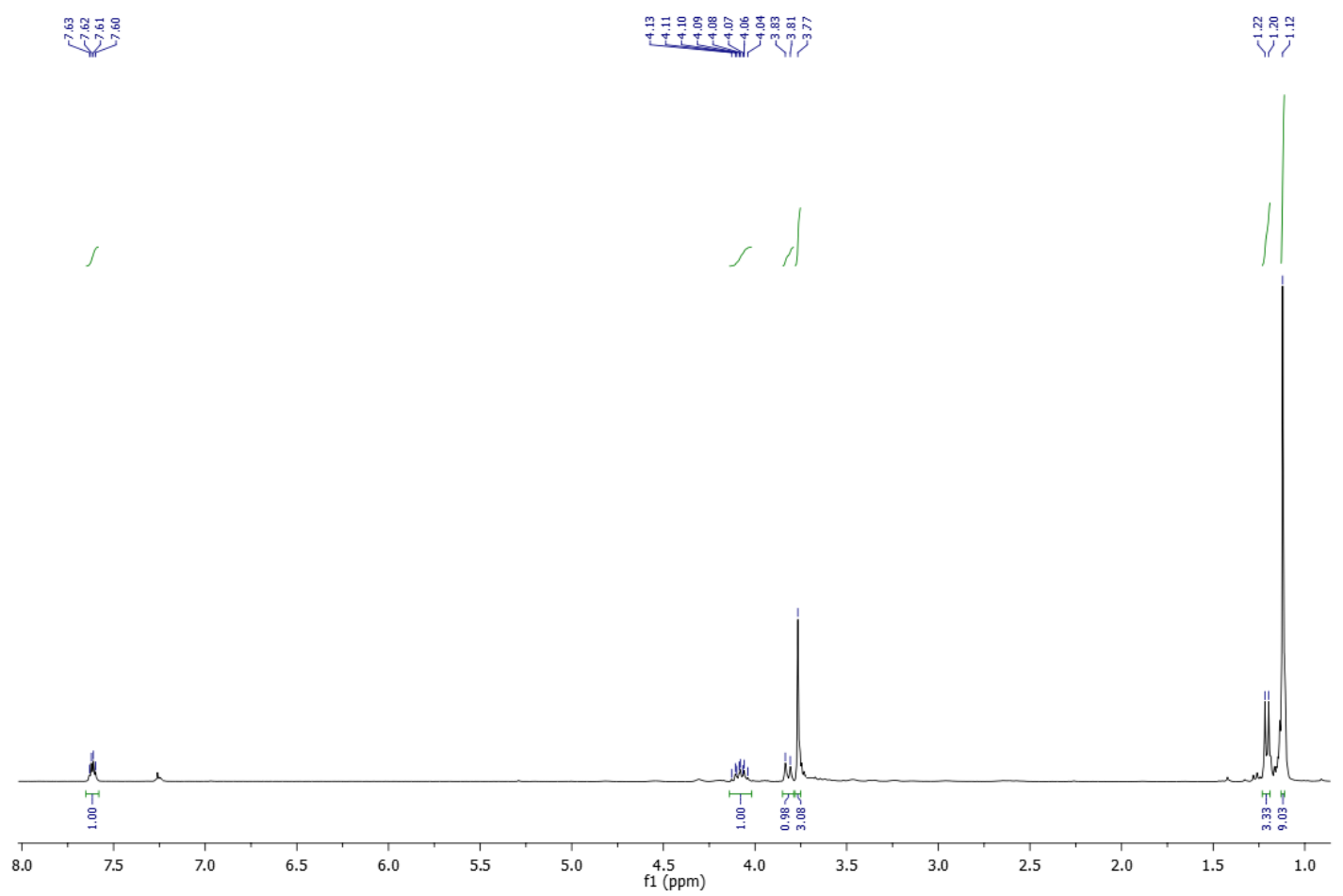




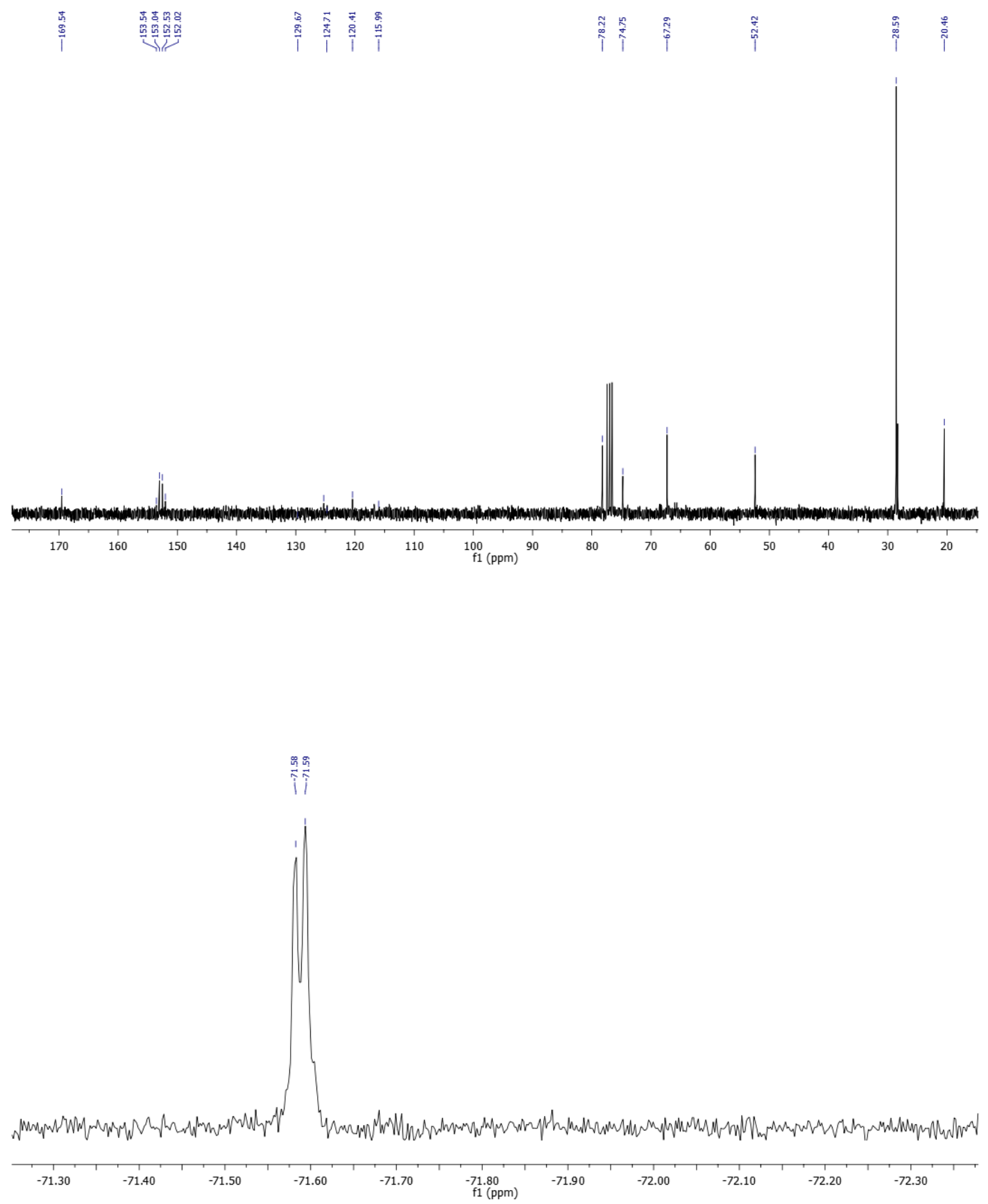


Methyl (2S,4S,5R)-5-methyl-2-(trifluoromethyl)-1,3-oxazolidine-4-carboxylate<smiles>COC(=O)[C@@H]1N[C@@H](C(F)(F)F)O[C@H]1C</smiles>

$11 f^{\prime}$
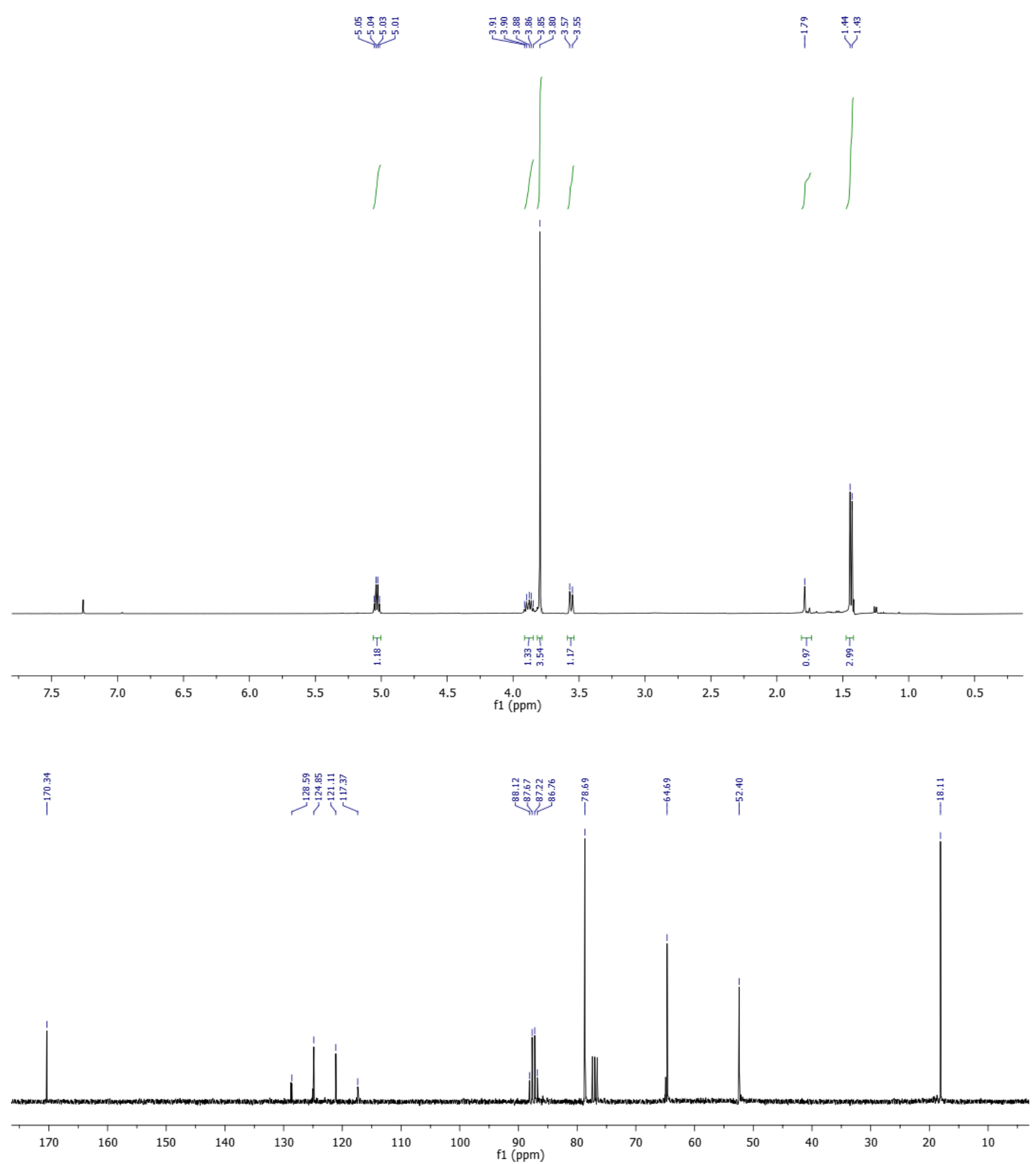


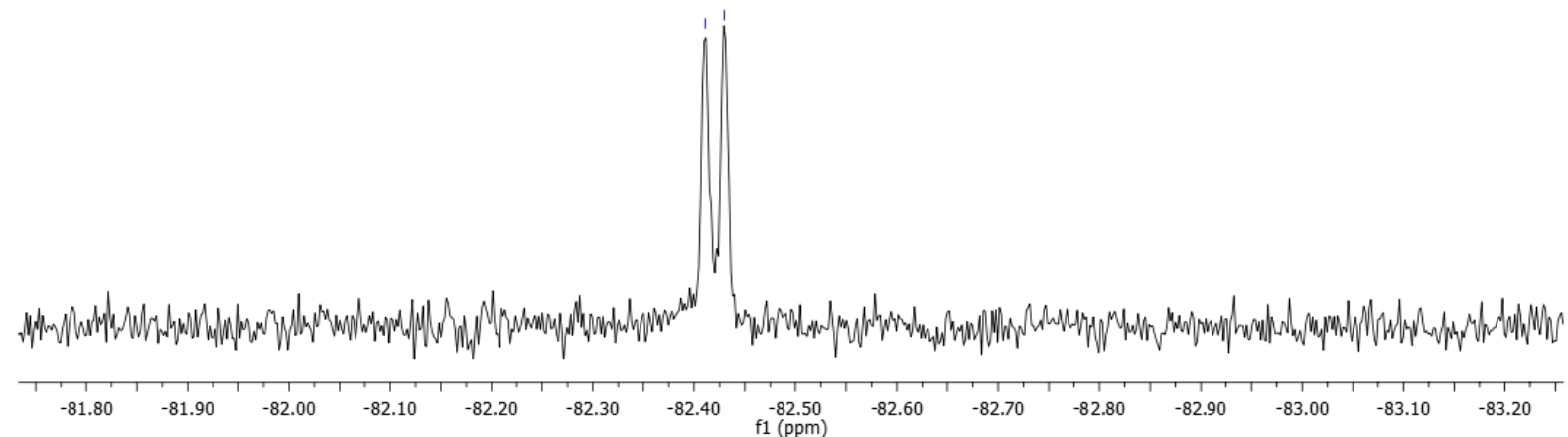


Ethyl 4,4,4-trifluoro-3-\{[(2S)-1-methoxy-3-methyl-1-oxobutan-2-yl]amino\}-2nitrobutanoate

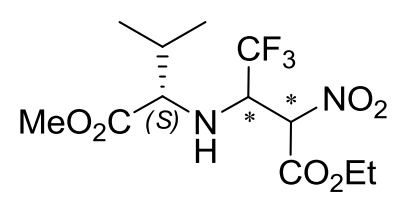

syn-12,13a, anti-12',13'a

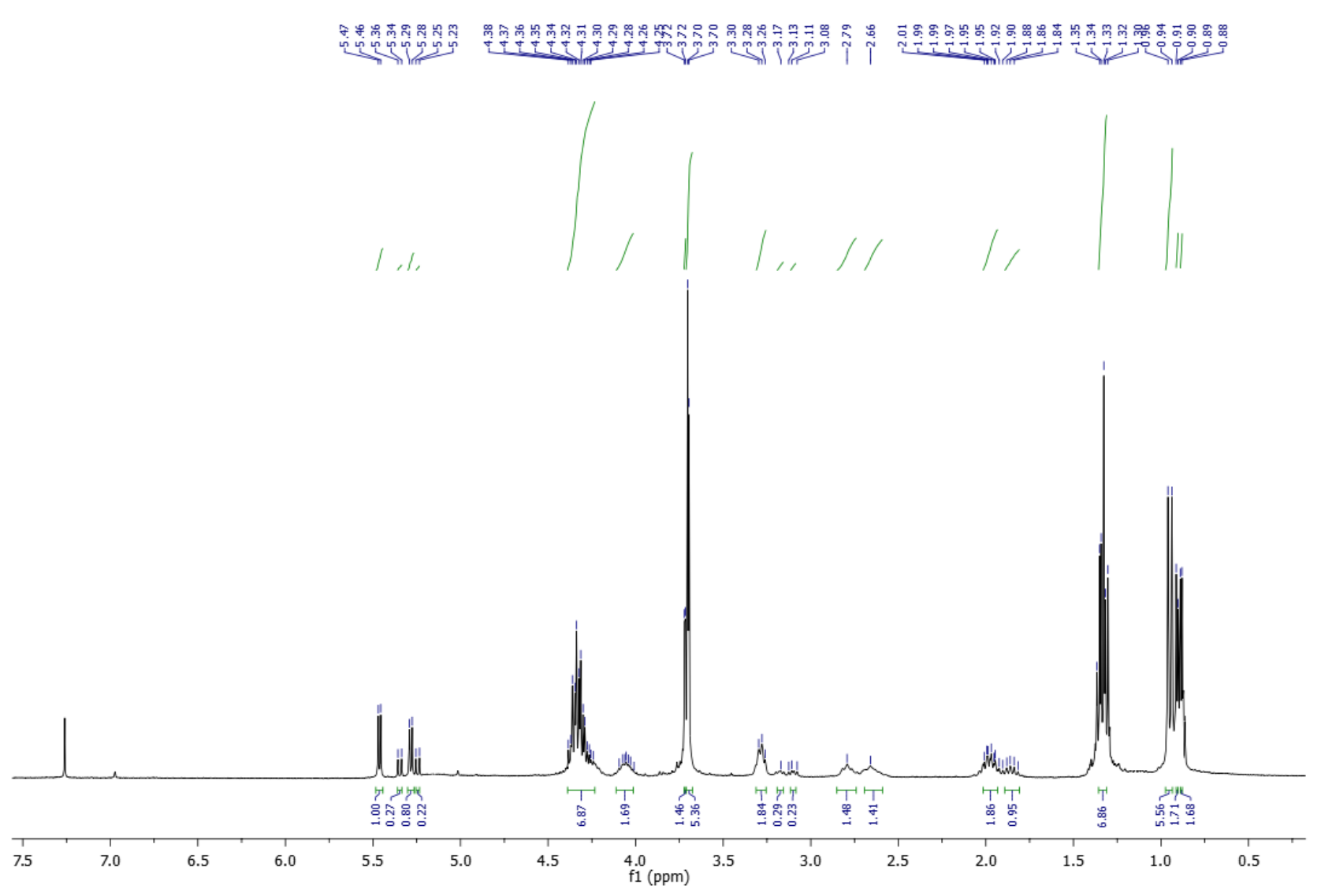



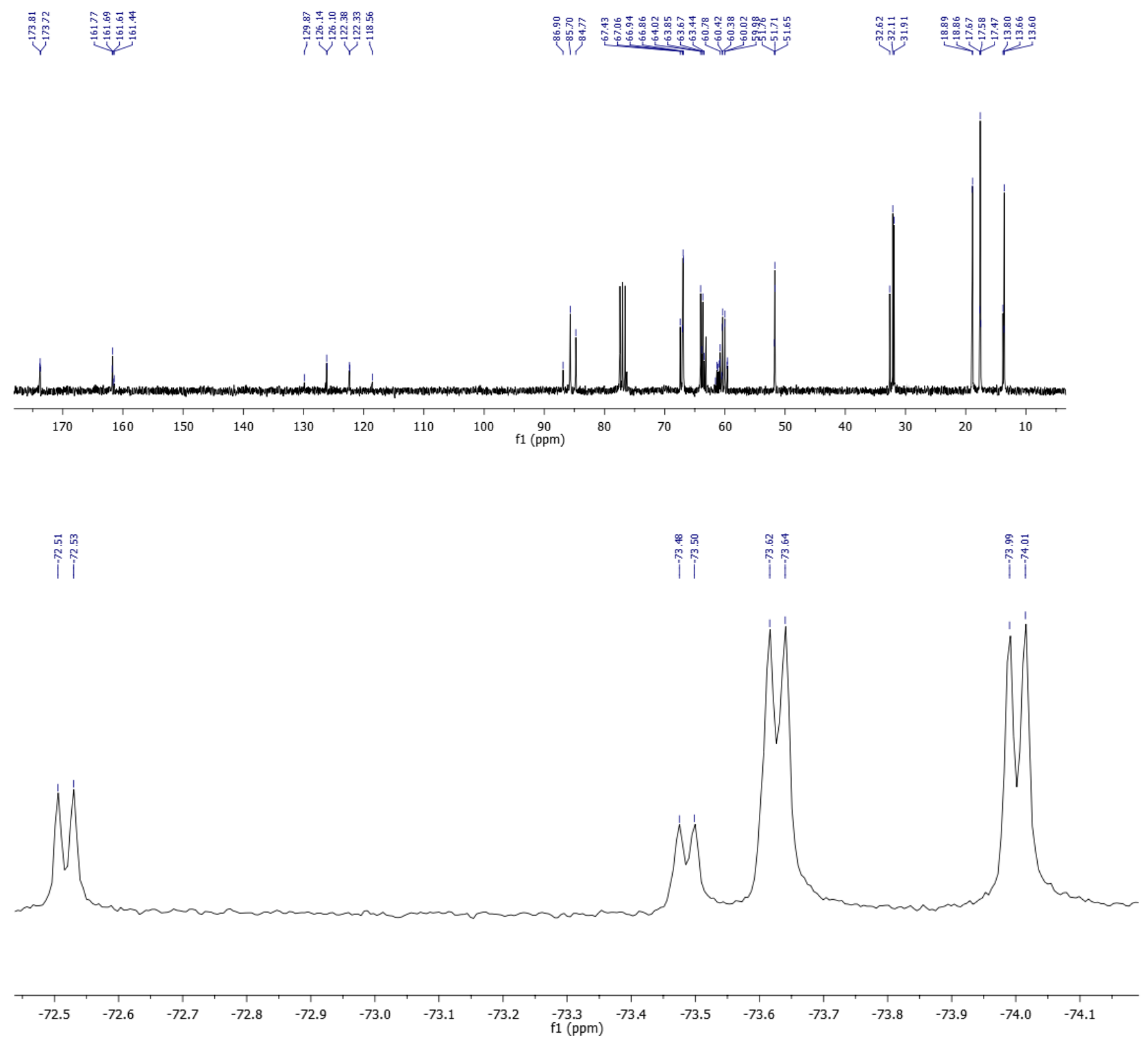
Methyl (2S,3S)-2-\{[4-ethoxy-1,1,1-trifluoro-3-nitro-4-oxobutan-2-yl]amino\}-3methylpentanoate

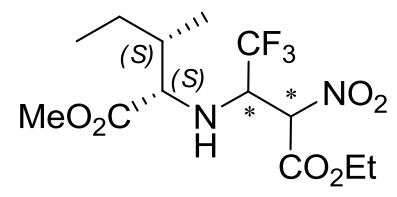

syn-12,13b, anti-12',13'b

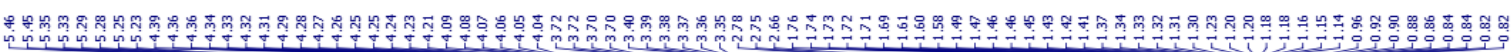
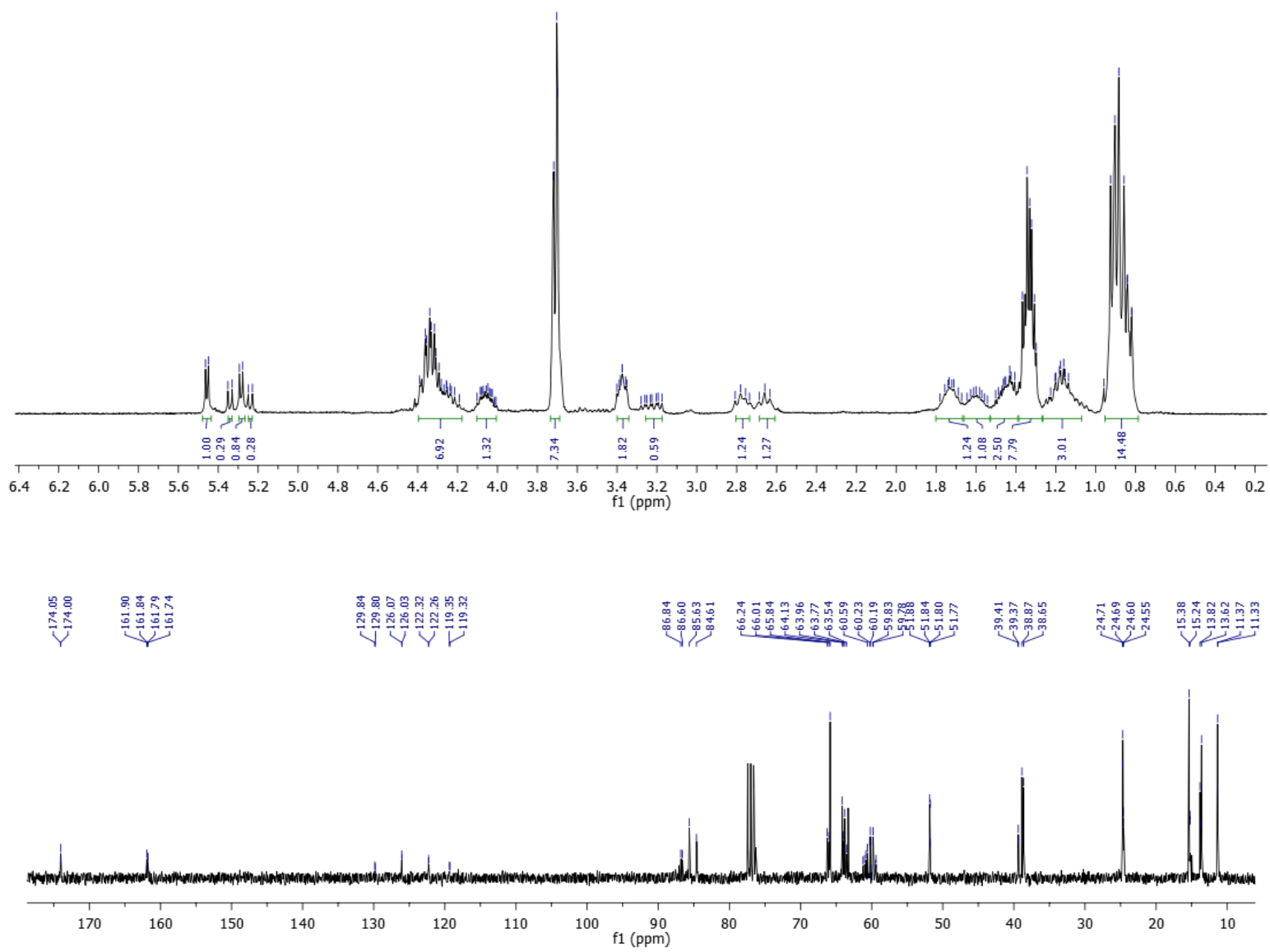


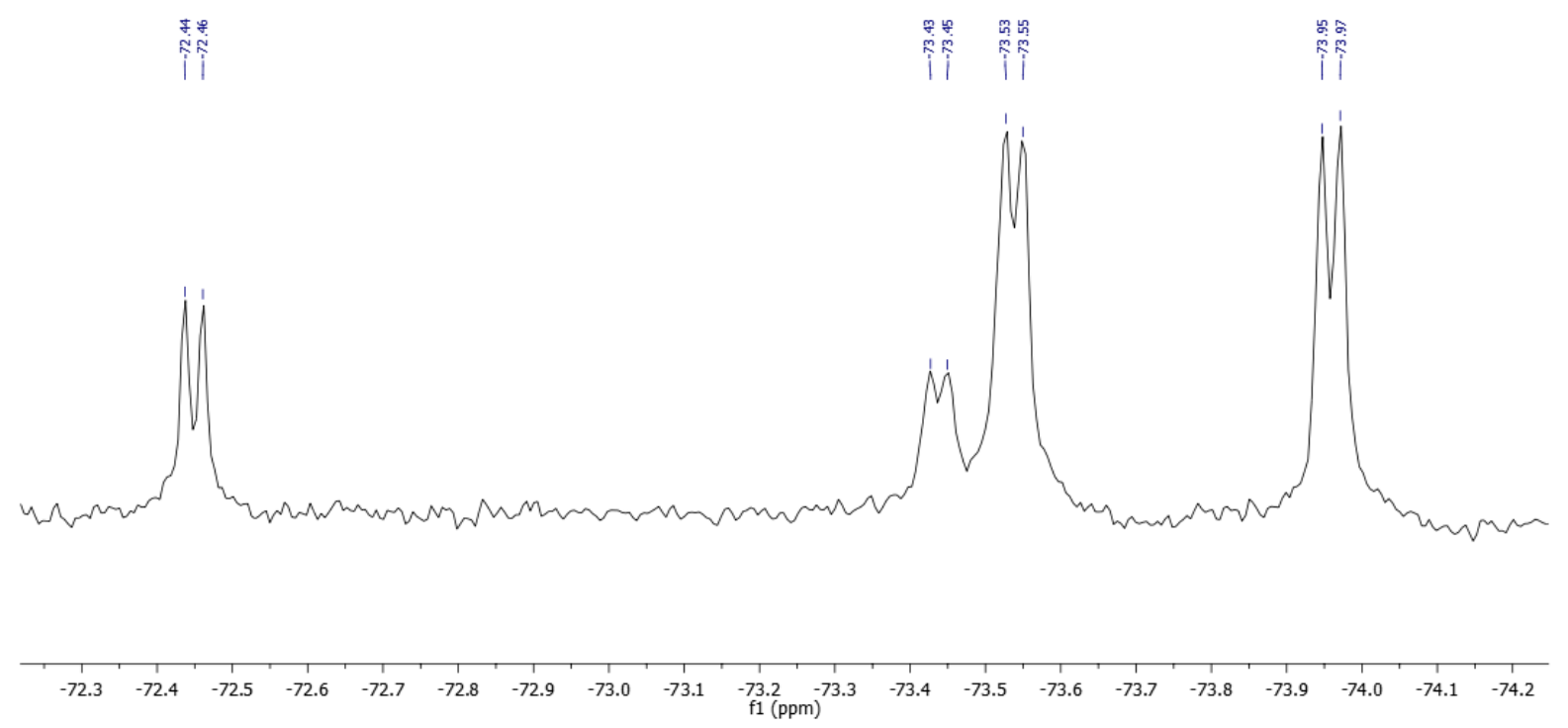


Methyl (2S)-2-\{[4-ethoxy-1,1,1-trifluoro-3-nitro-4-oxobutan-2-yl]amino\}-4methylpentanoate<smiles>CCOC(=[N+]([O-])C(=O)[N+](=O)[O-])C(CC(C)=O)(CC(C)C)C(F)(F)F</smiles>

syn-12,13c, anti-12',13'c

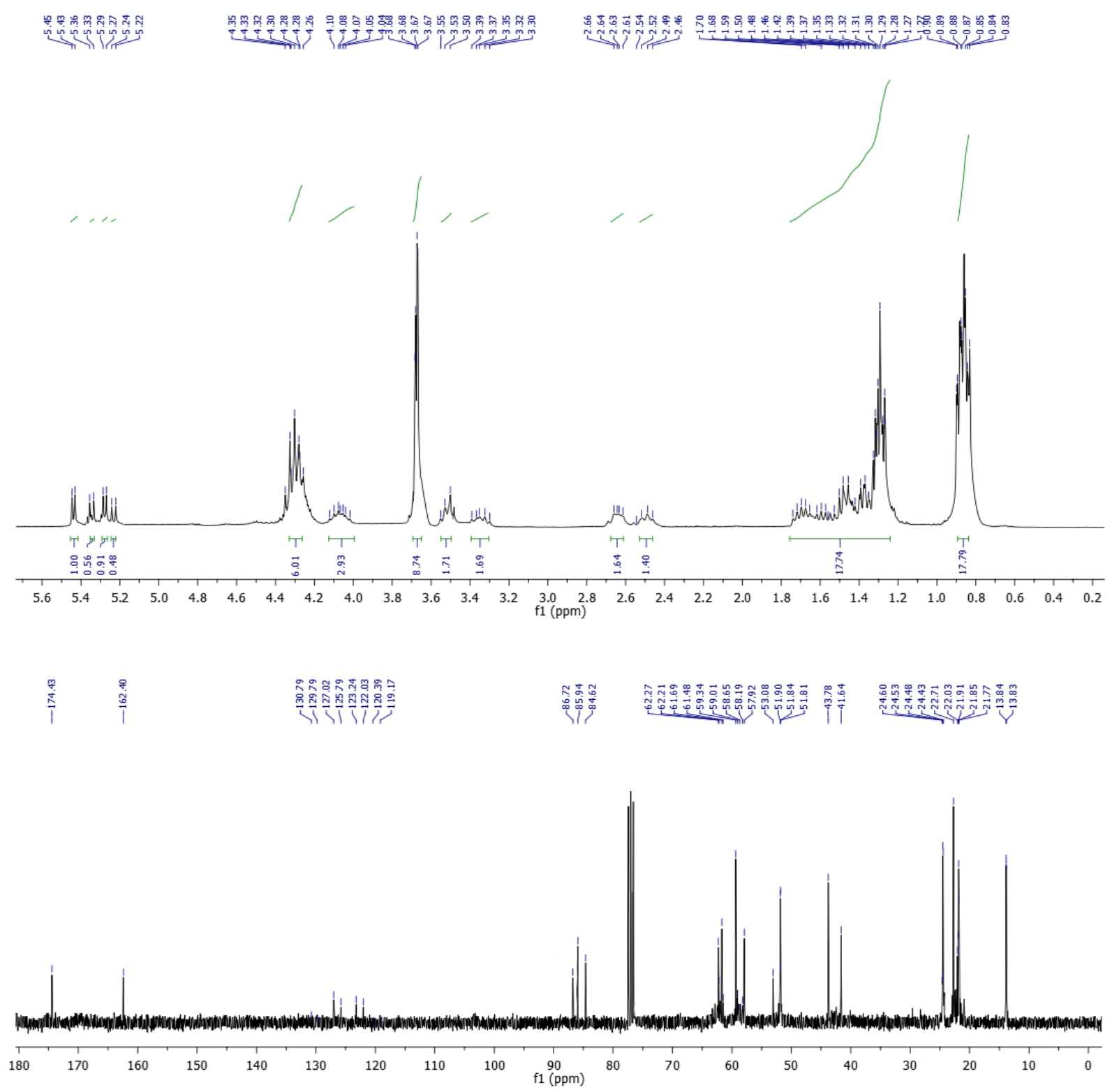




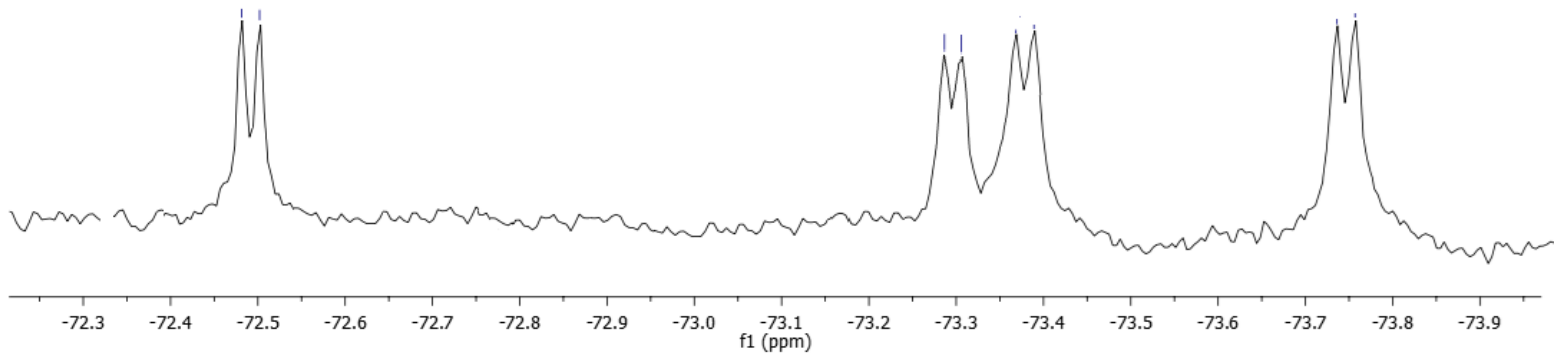


Ethyl 4,4,4-trifluoro-3-\{[(2S)-1-methoxy-4-(methylsulfanyl)-1-oxobutan-2-yl]amino\}-2nitrobutanoate<smiles>CCOC(=O)C(OCC)=C(N[C@@H](CCSC)C(C)=O)C(F)(F)F</smiles>

syn-12,13d, anti-12',13'd

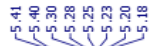

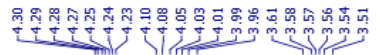

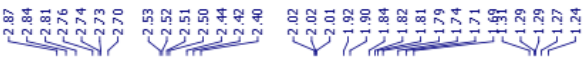

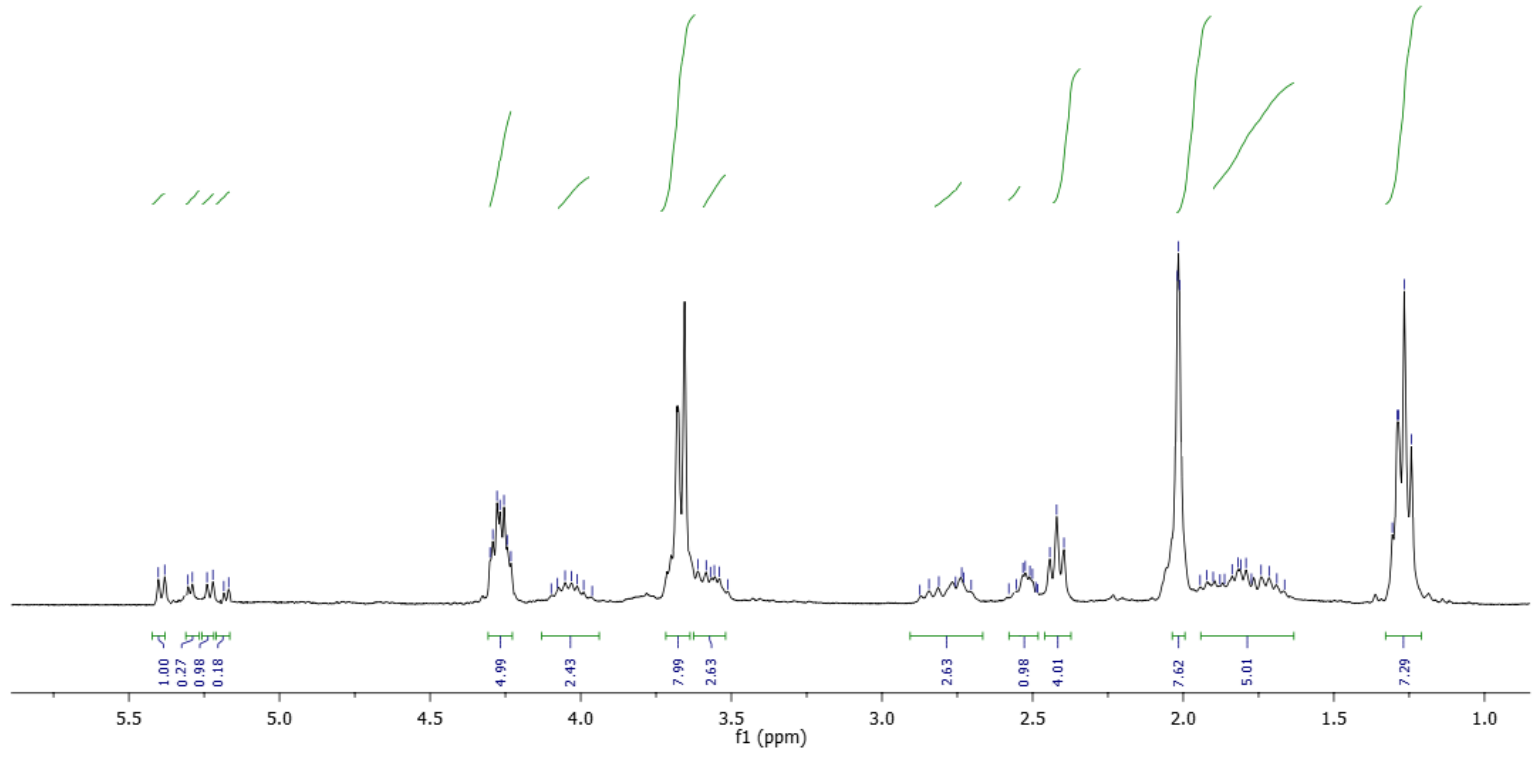




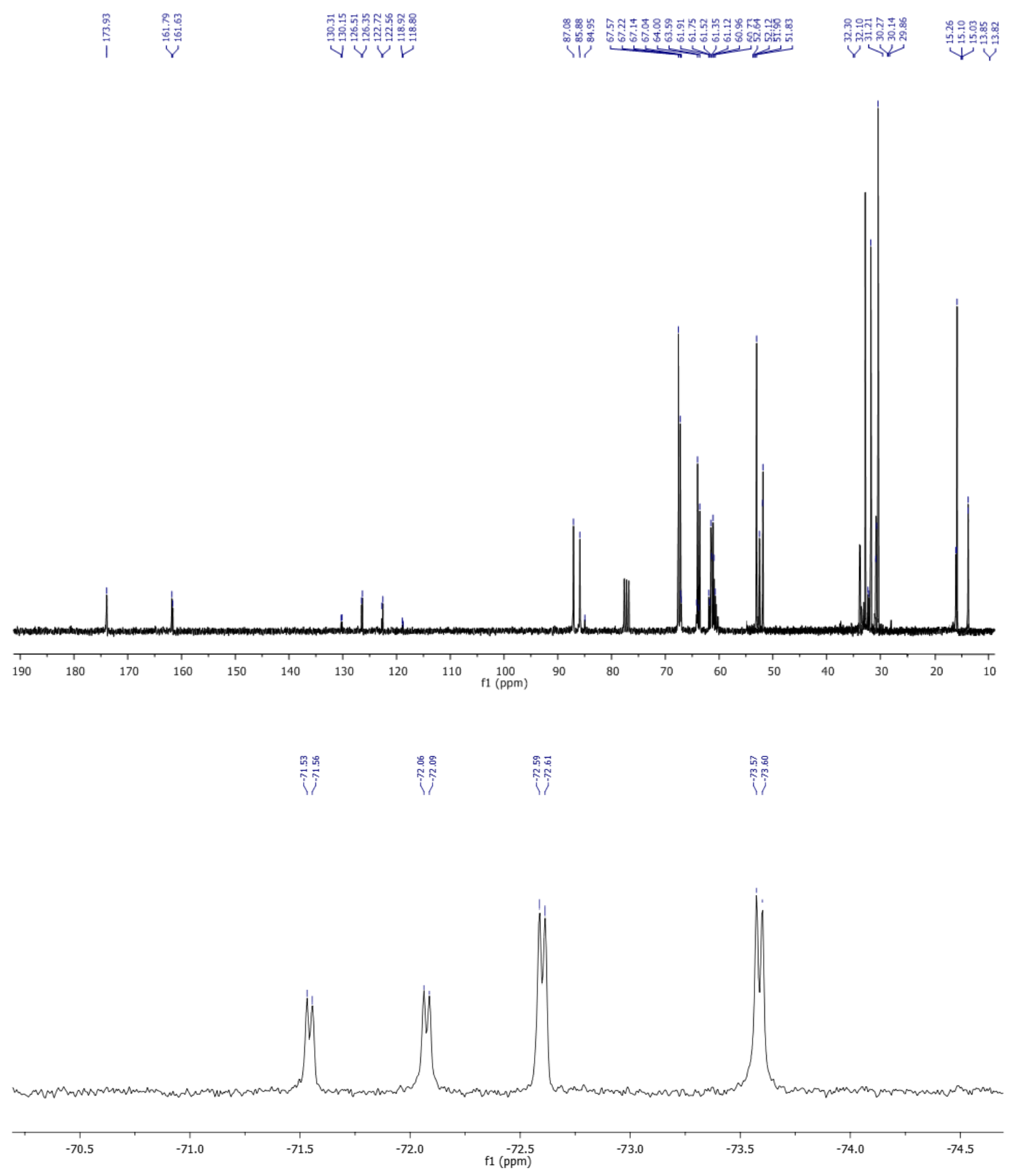


Ethyl 3-amino-4,4,4-trifluoro-2-nitrobutanoate<smiles>CCOC(=O)C(=C(N)C(F)(F)F)[N+](=O)[O-]</smiles>

syn-14, anti-14'
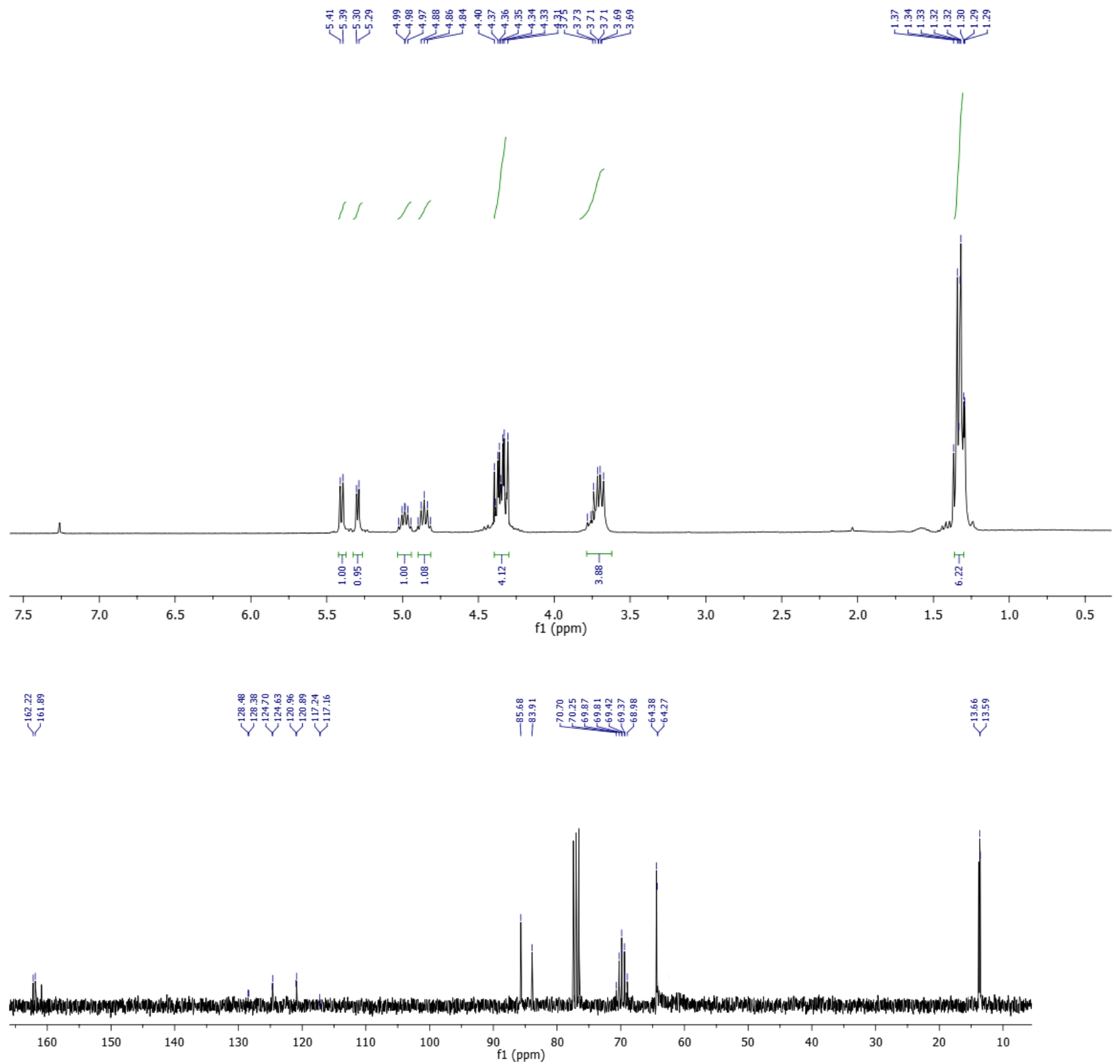


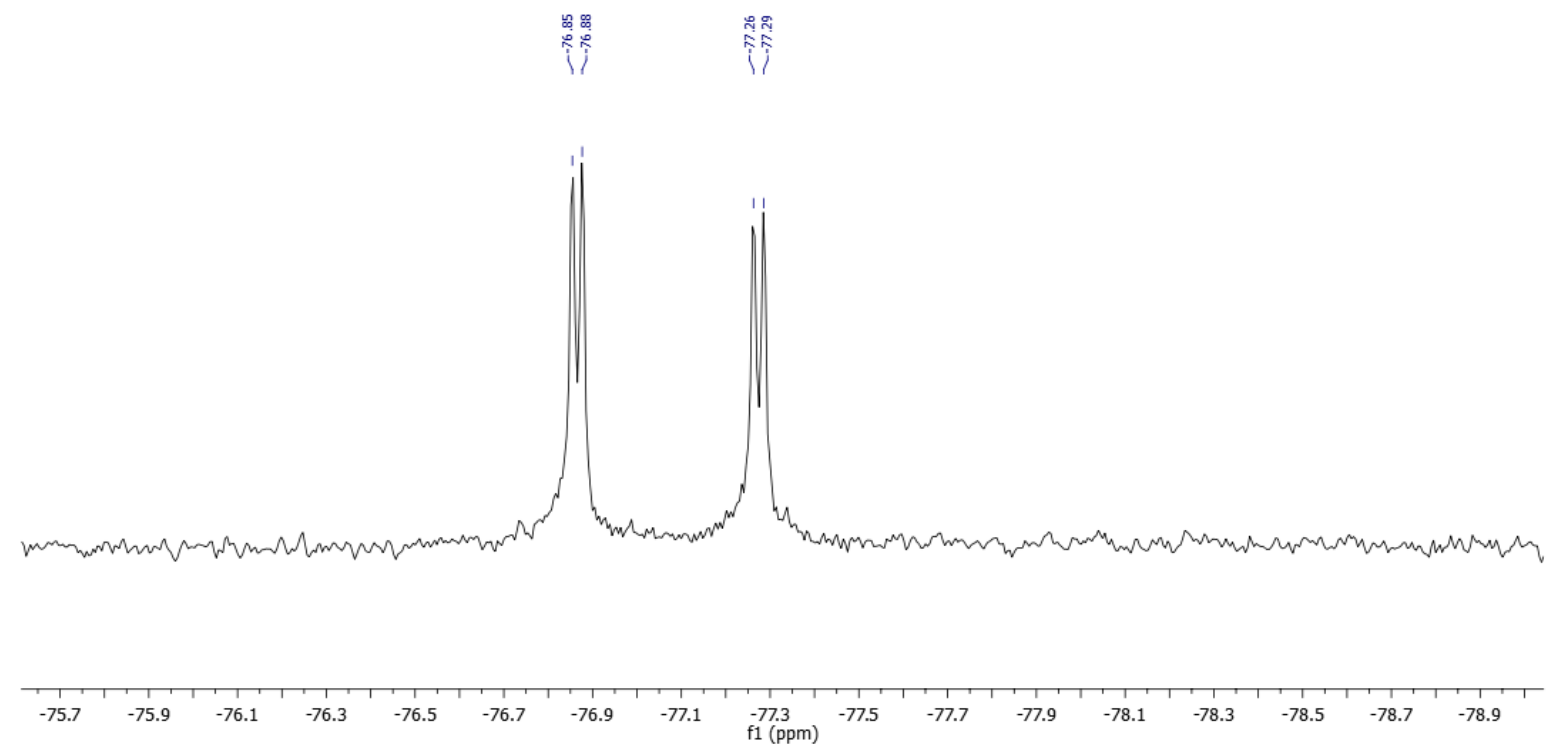


Ethyl (2S,3R)-2-amino-4,4,4-trifluoro-3-\{[(2S)-1-methoxy-3-methyl-1-oxobutan-2yl]]amino\}butanoate

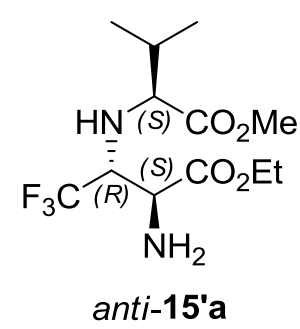

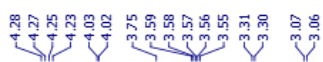

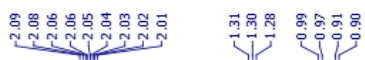
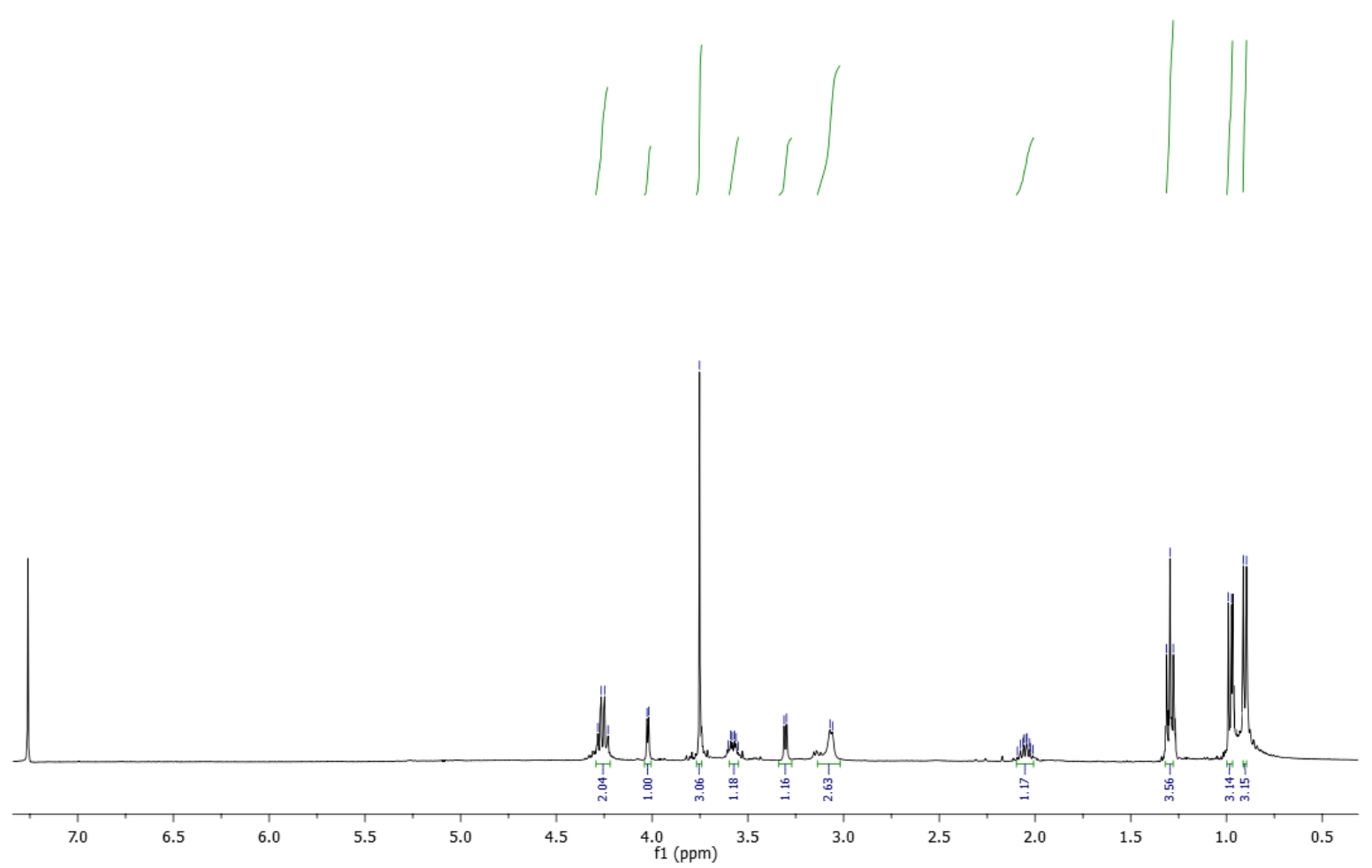


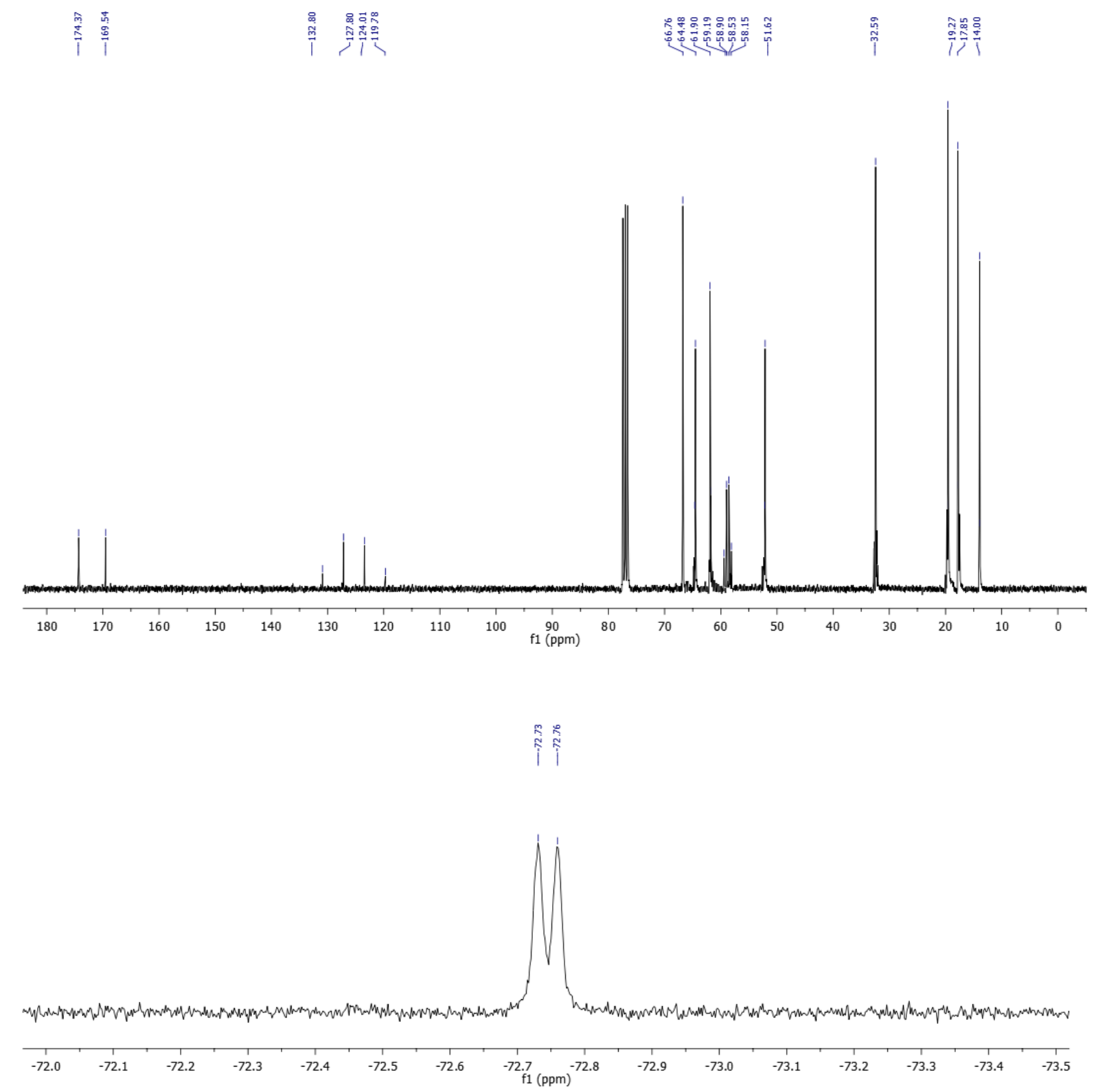


Ethyl (2R,3S)-2-amino-4,4,4-trifluoro-3-\{[(2S)-1-methoxy-3-methyl-1-oxobutan-2yl]amino\}butanoate

$$
\text { anti-16'a }
$$
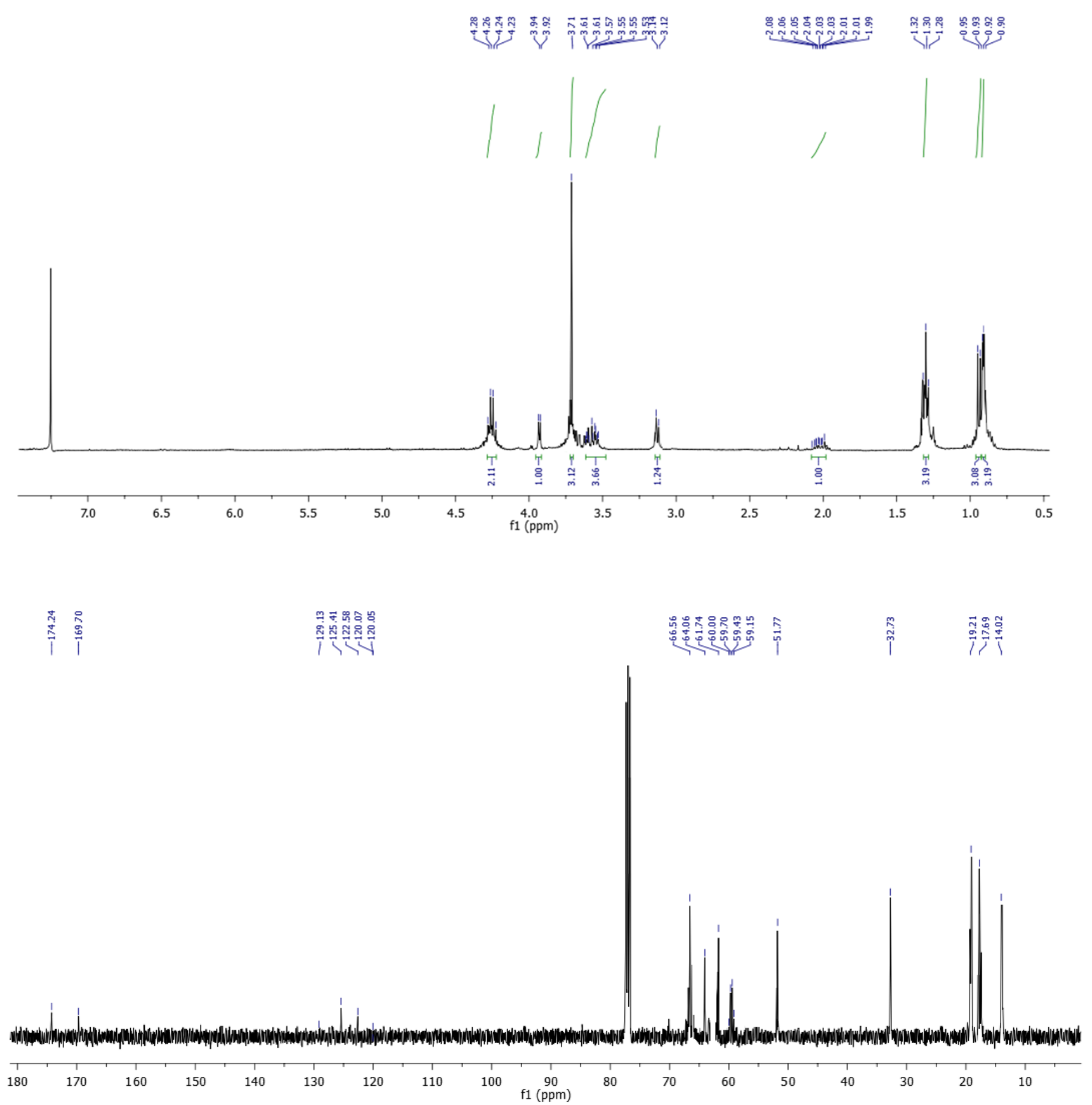


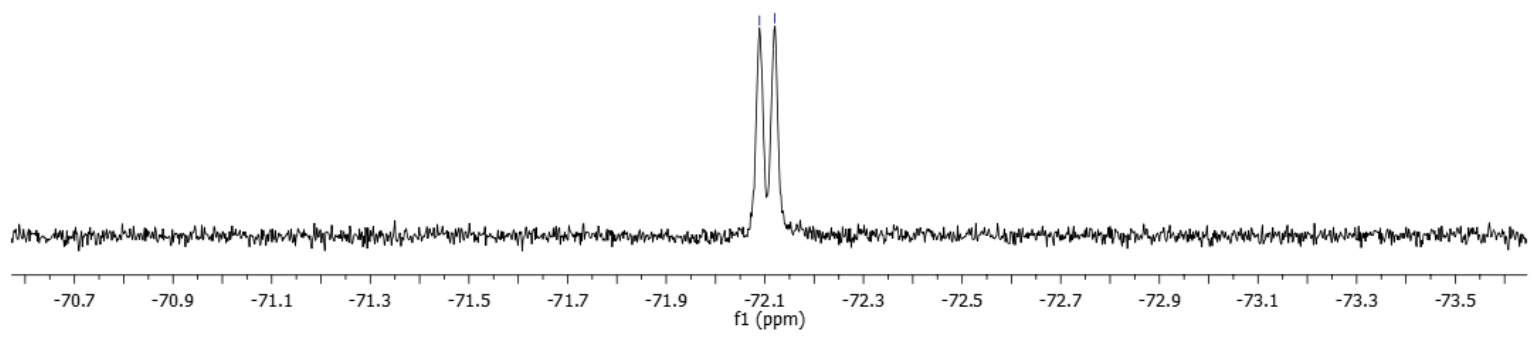


Methyl (2S,3S)-2-\{[(2R,3S)-3-amino-4-ethoxy-1,1,1-trifluoro-4-oxobutan-2-yl]amino\}-3methylpentanoate<smiles>CCOC(=O)N[C@H](C(N)C(=O)OCC)C(F)(F)F</smiles>

anti-15'b

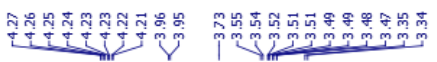

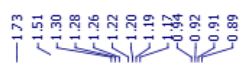

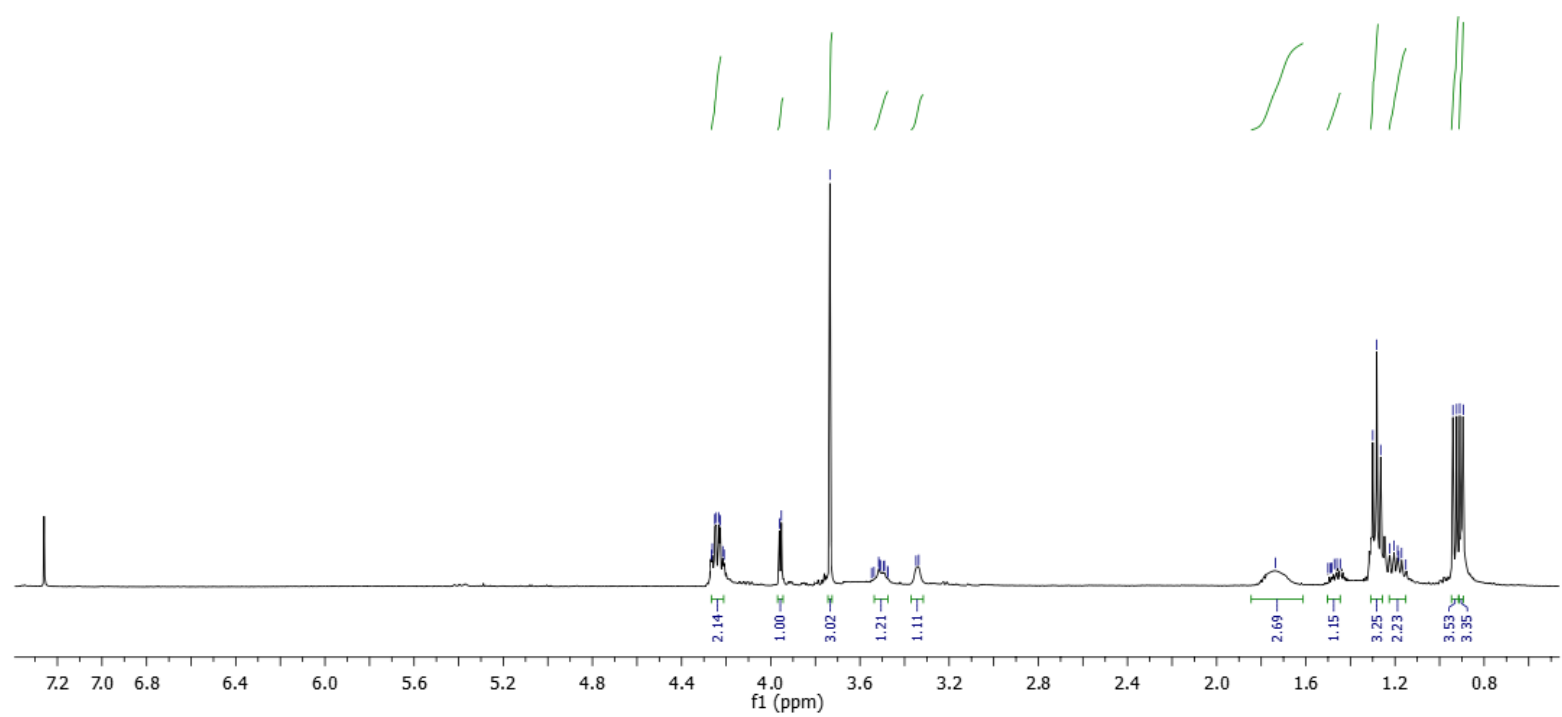

里

烈獎

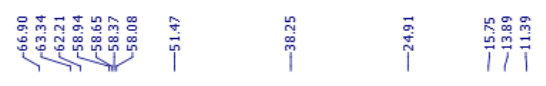

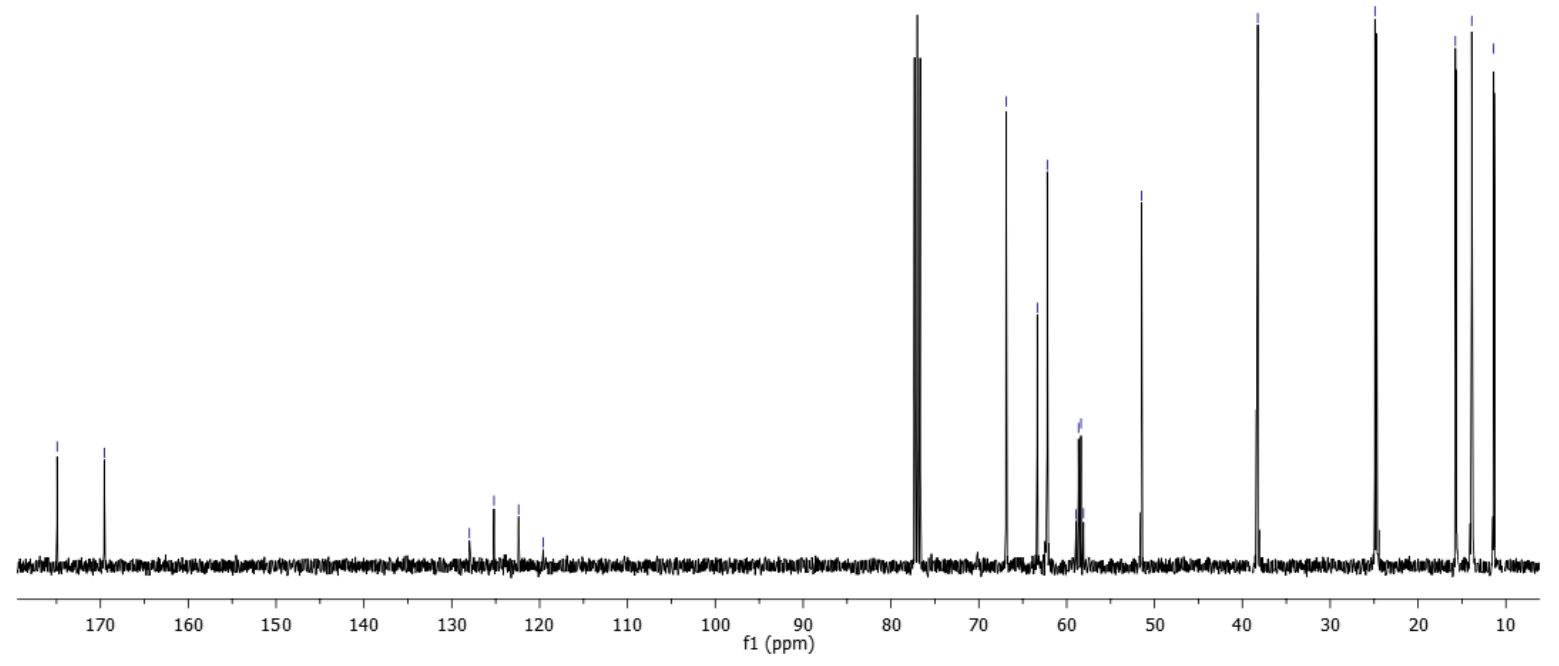




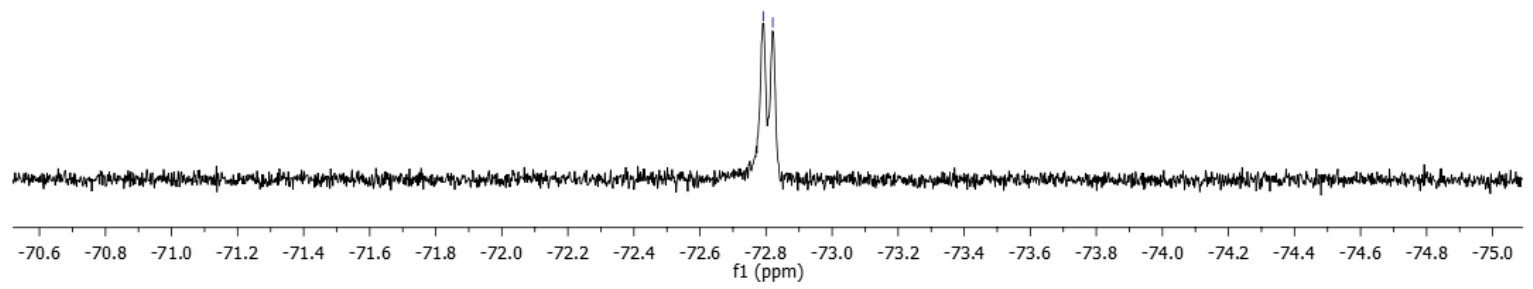


Methyl (2S,3S)-2-\{[(1S,2R)-2-amino-4-ethoxy-1,1,1-trifluoro-4-oxobutan-2-yl]amino\}-3methylpentanoate<smiles>CCOC(=O)C(N)C(NC(C(=O)OCC)C(F)(F)F)C(F)(F)F</smiles>

anti-16'b
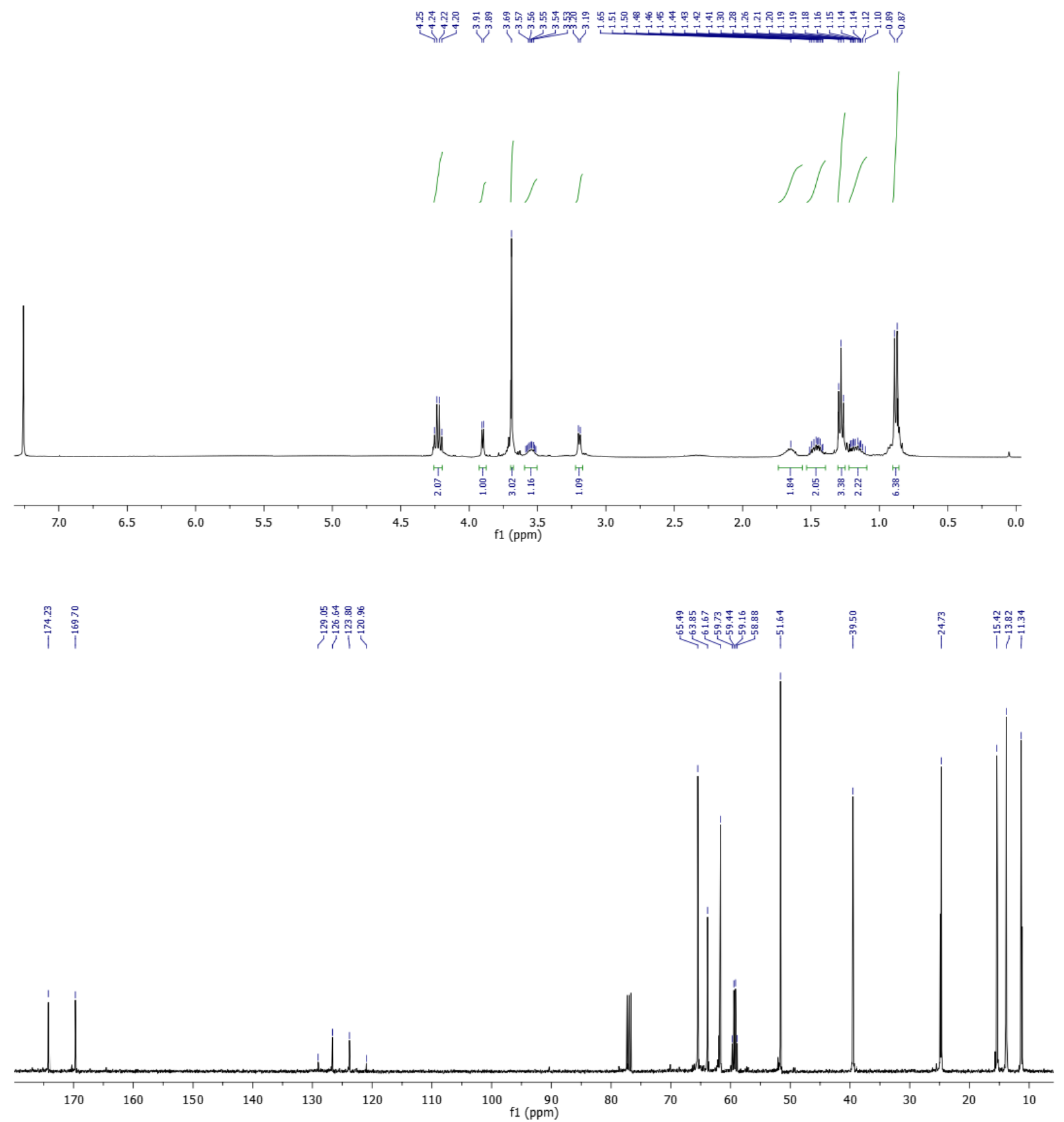
4.

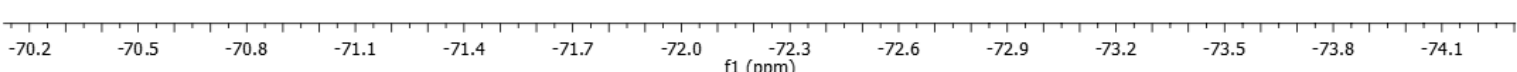

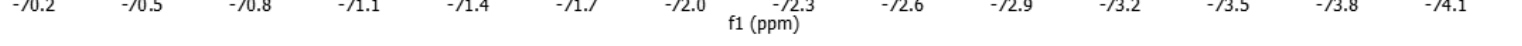


Methyl (2S)-2-\{[(2R,3S)-3-amino-4-ethoxy-1,1,1-trifluoro-4-oxobutan-2-yl]amino\}-4methylpentanoate

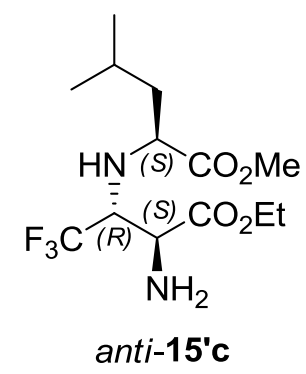

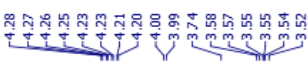

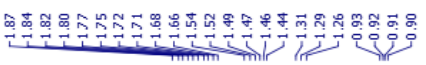
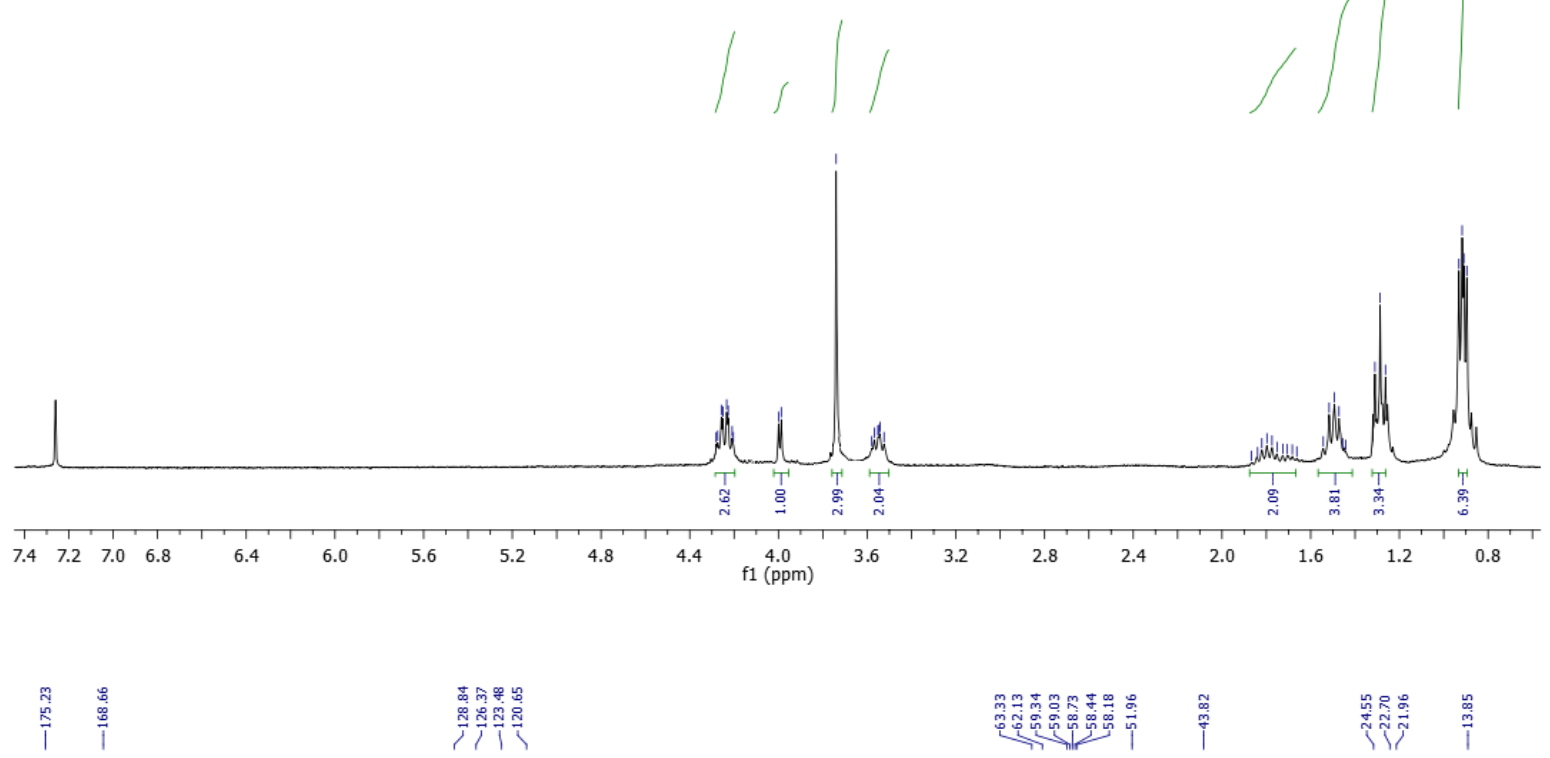

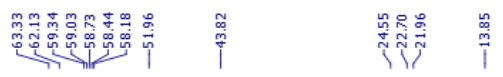

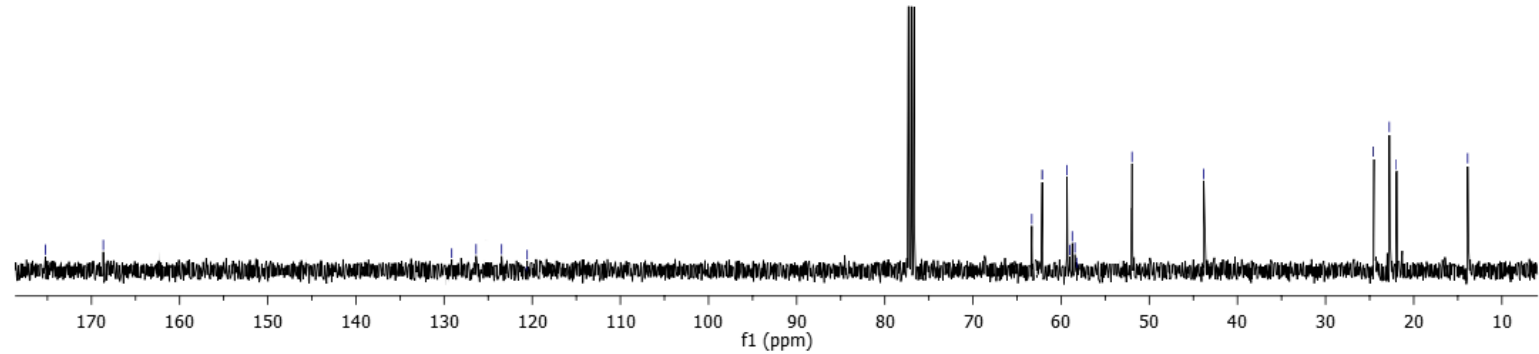




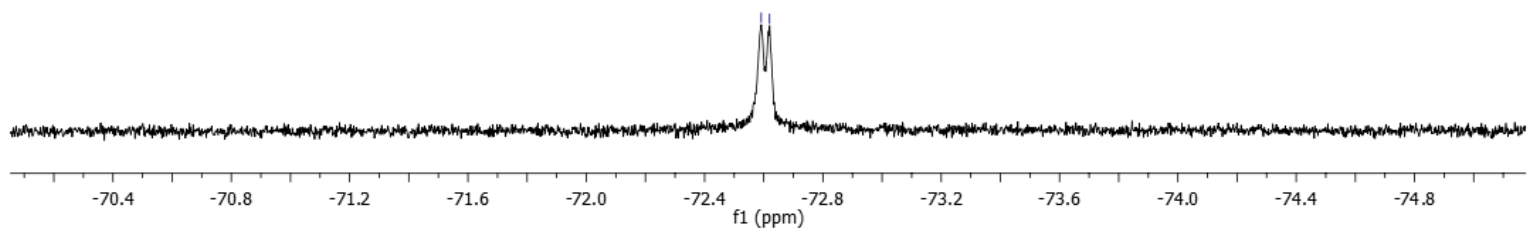


Methyl (2S)-2-\{[(2S,3R)-3-amino-4-ethoxy-1,1,1-trifluoro-4-oxobutan-2-yl]amino\}-4methylpentanoate

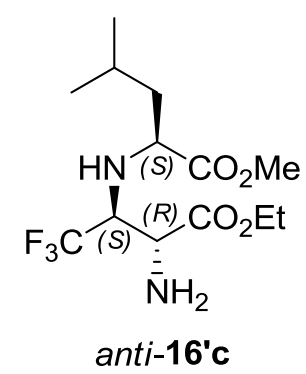

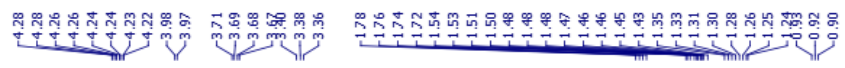

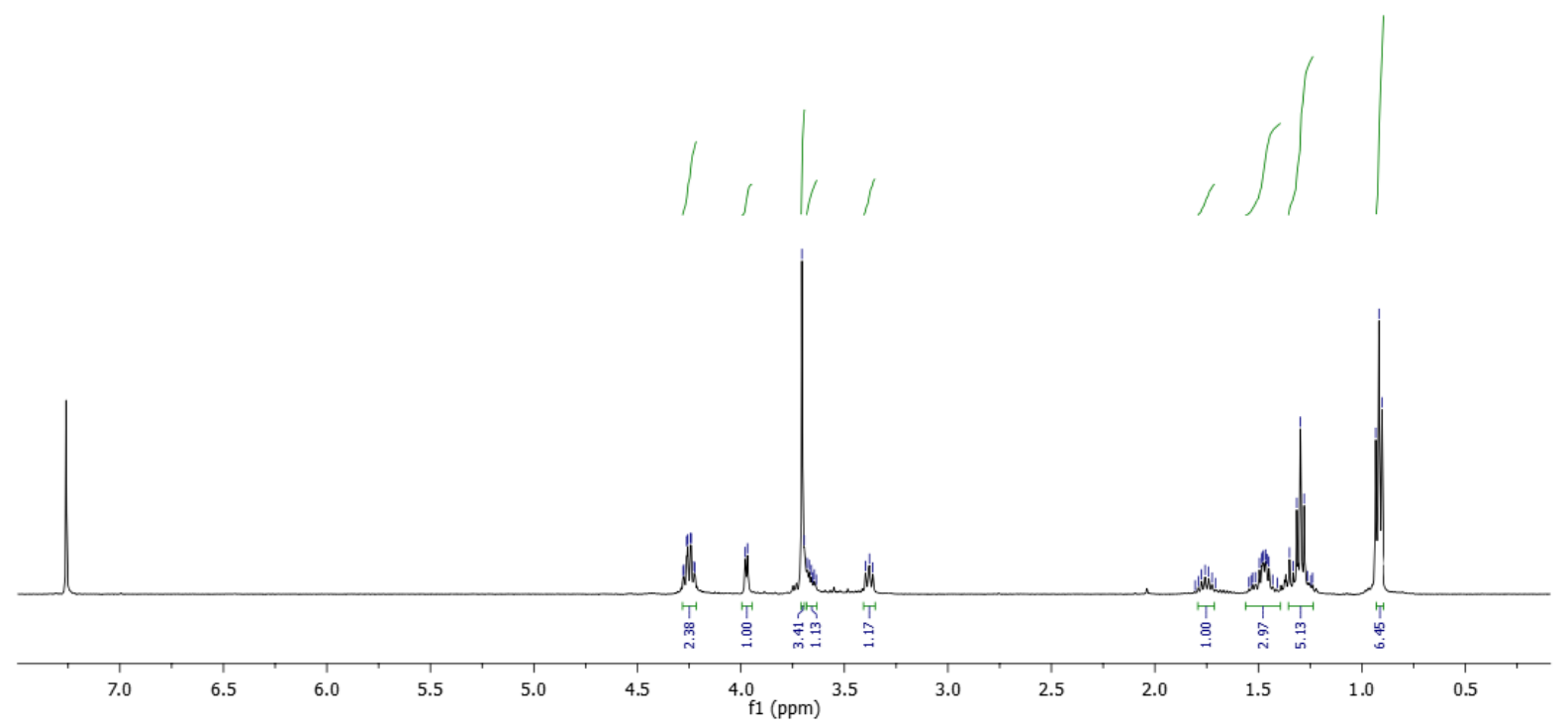

細

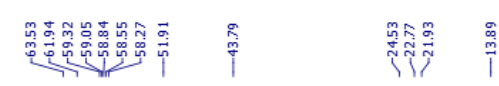

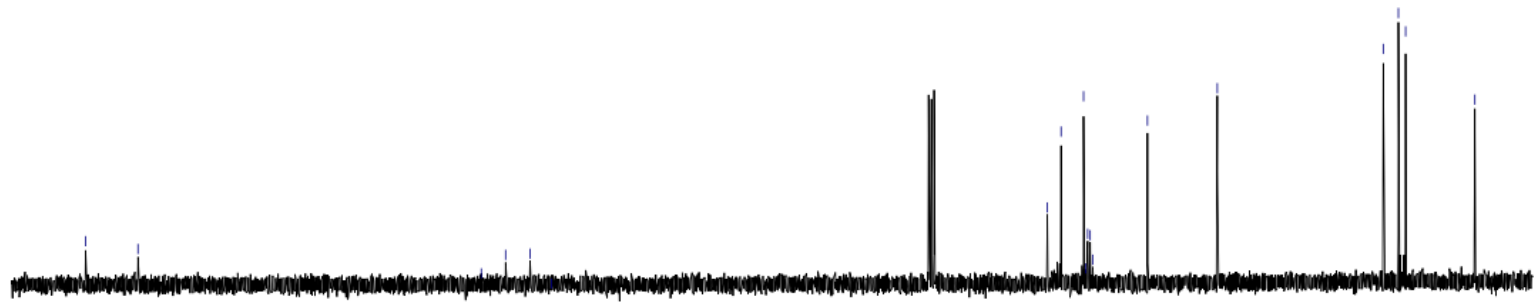

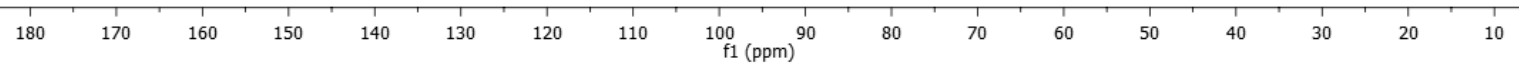




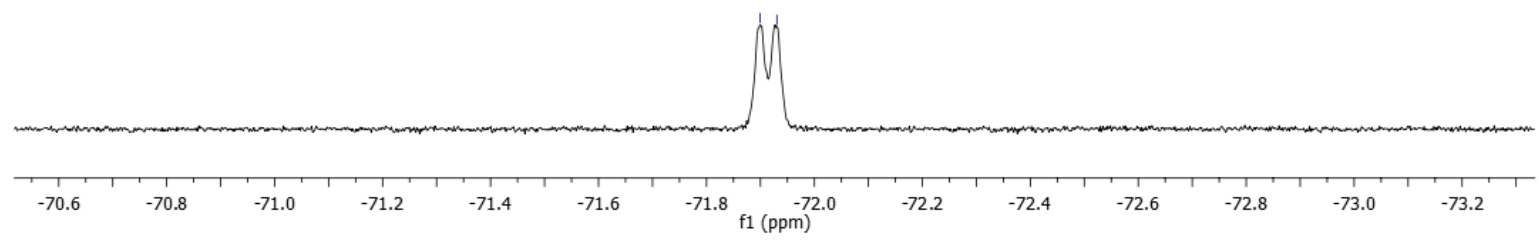


Ethyl (3R)-4,4,4-trifluoro-2-imino-3-\{[(2S)-1-methoxy-3-methyl-1-oxobutan-2yl]amino\}butanoate<smiles>CCOC(=N)C(NC(C(=O)OCC)C(C)C)C(F)(F)F</smiles>

$17 a$

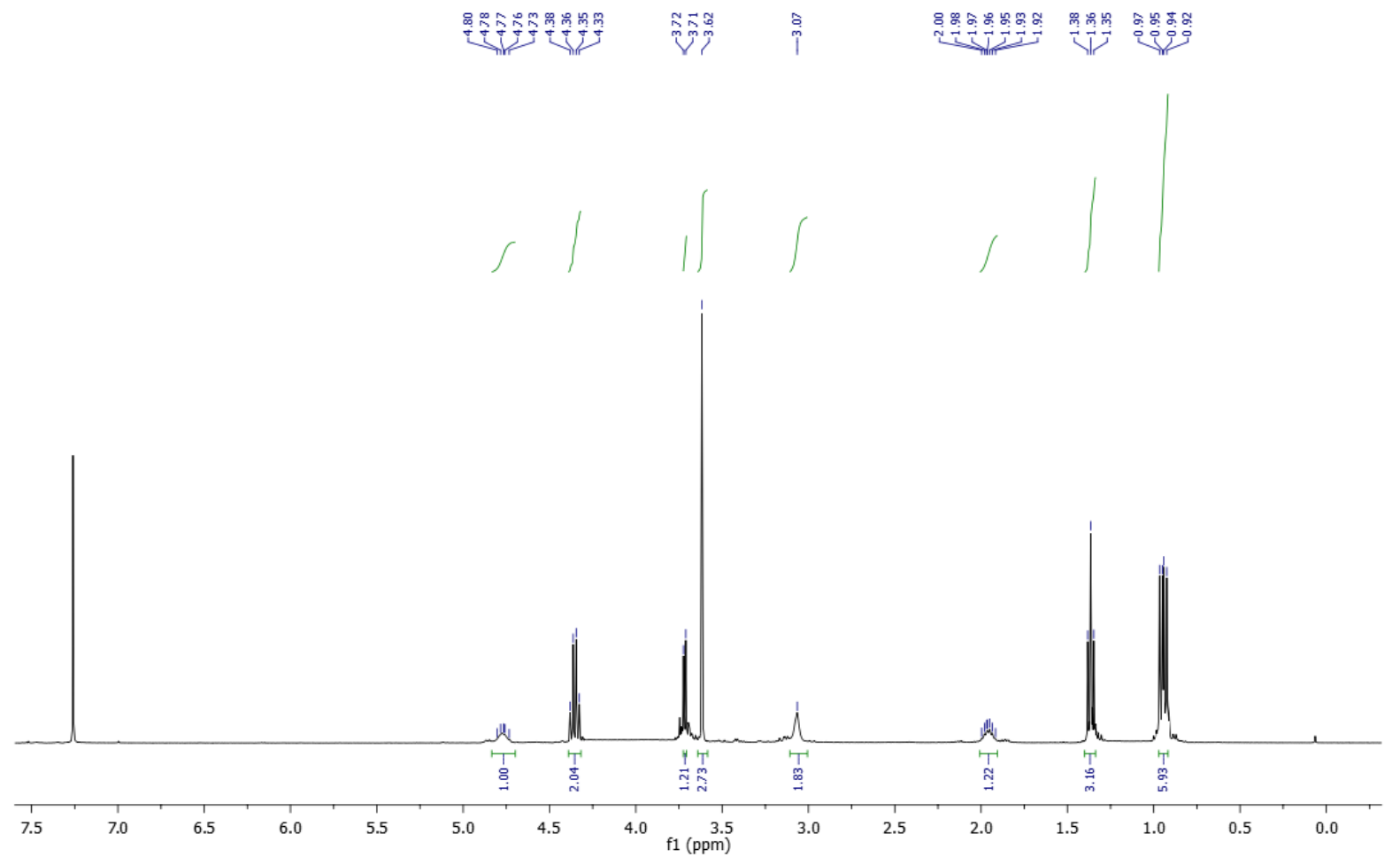

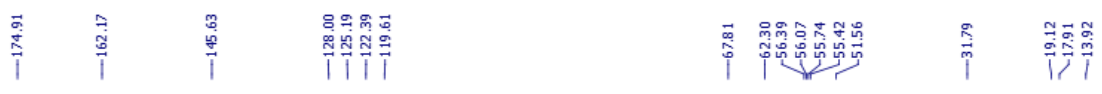

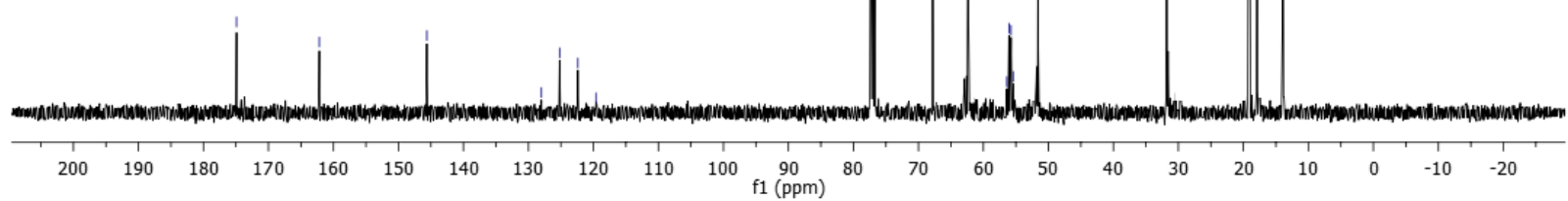




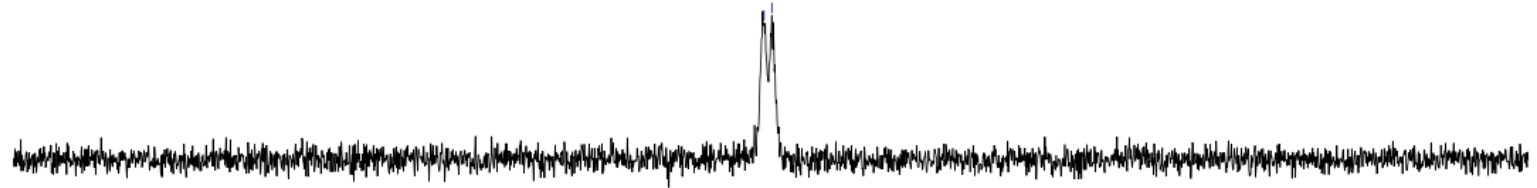

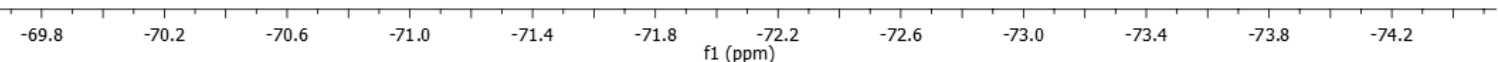


Ethyl (3S)-4,4,4-trifluoro-2-imino-3-\{[(2S)-1-methoxy-3-methyl-1-oxobutan-2yl]amino\}butanoate<smiles>CCOC(=N)C(NC(C(=O)OCC)C(C)C)C(F)(F)F</smiles>

$18 \mathrm{a}$
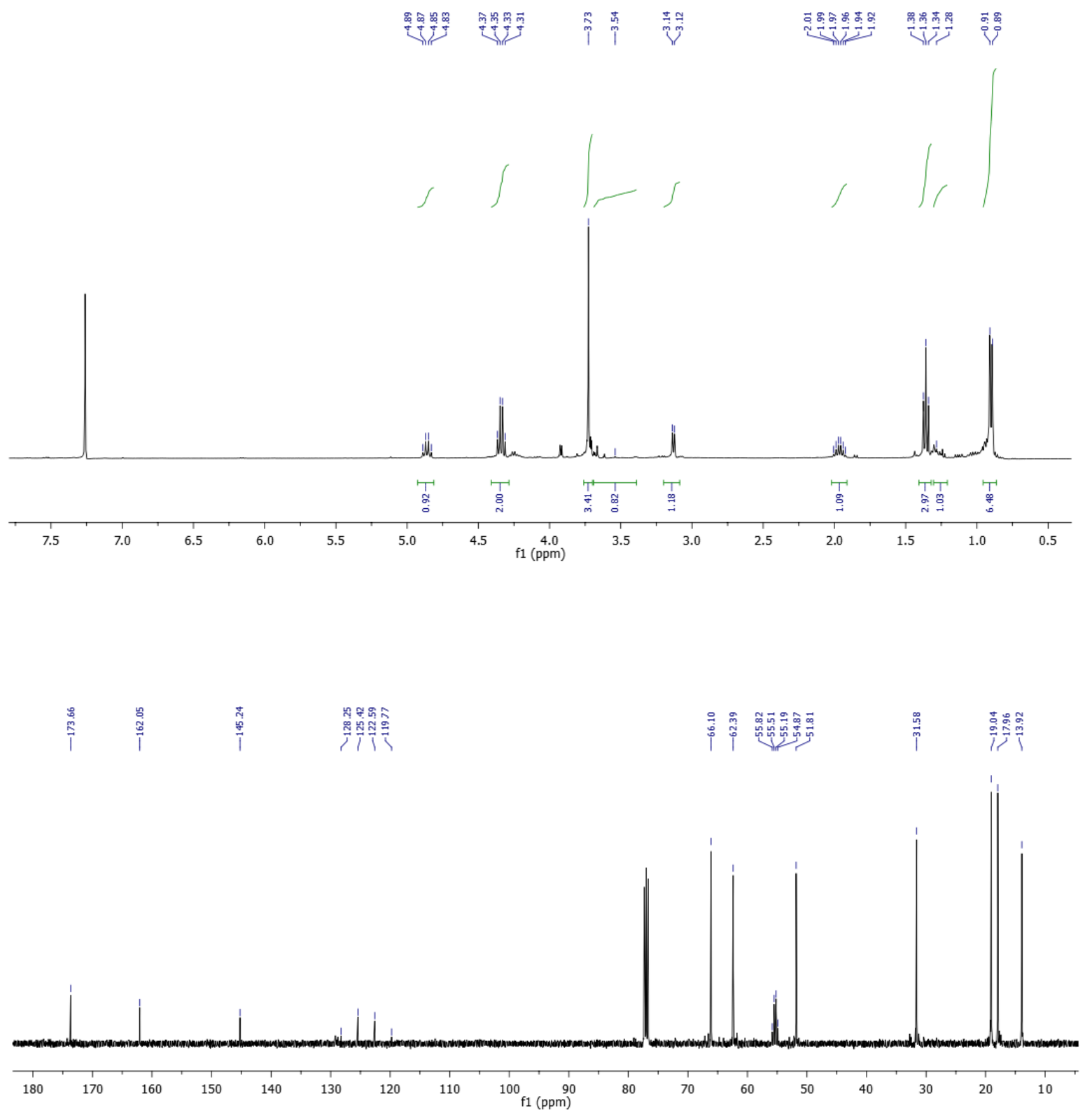


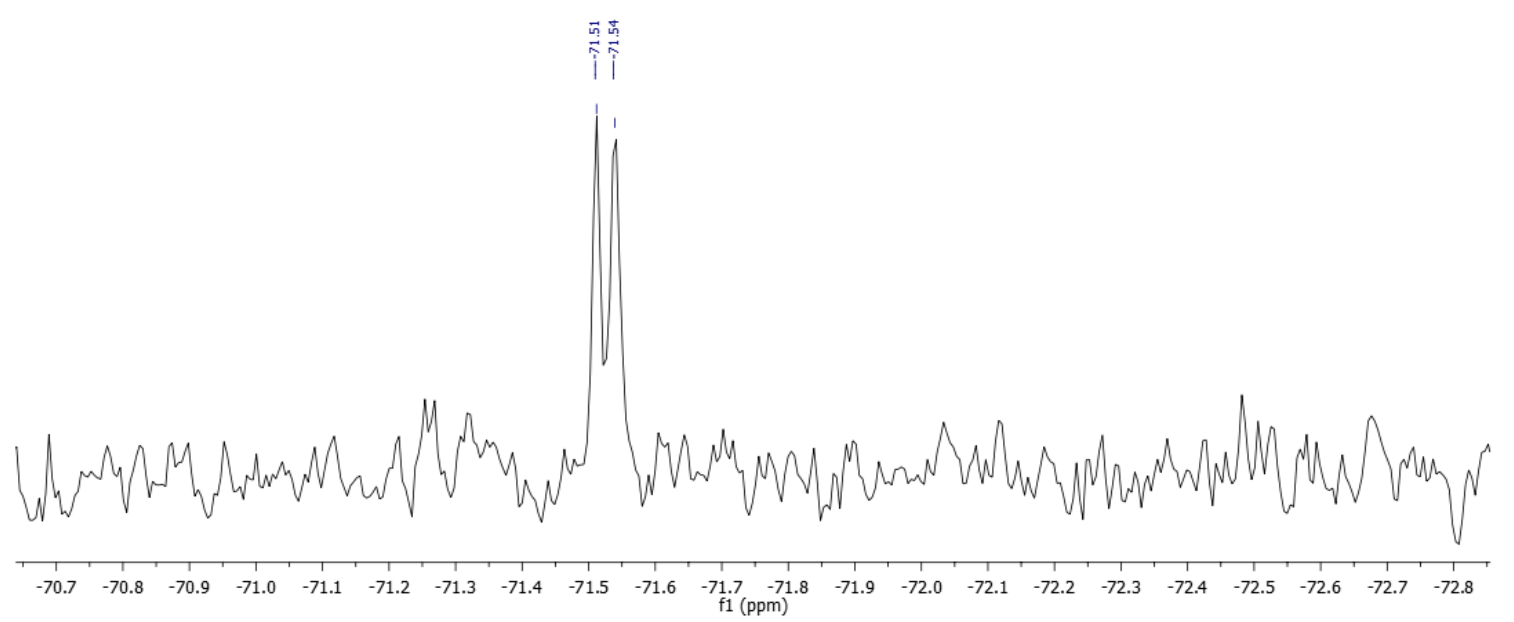


Methyl (2S,3S)-2-\{[(2R)-4-ethoxy-1,1,1-trifluoro-3-imino-4-oxobutan-2-yl]amino\}-3methylpentanoate<smiles>CCOC(=N)C(NC(C(=O)OCC)C(F)(F)C(F)(F)F)C(=O)OCC</smiles>
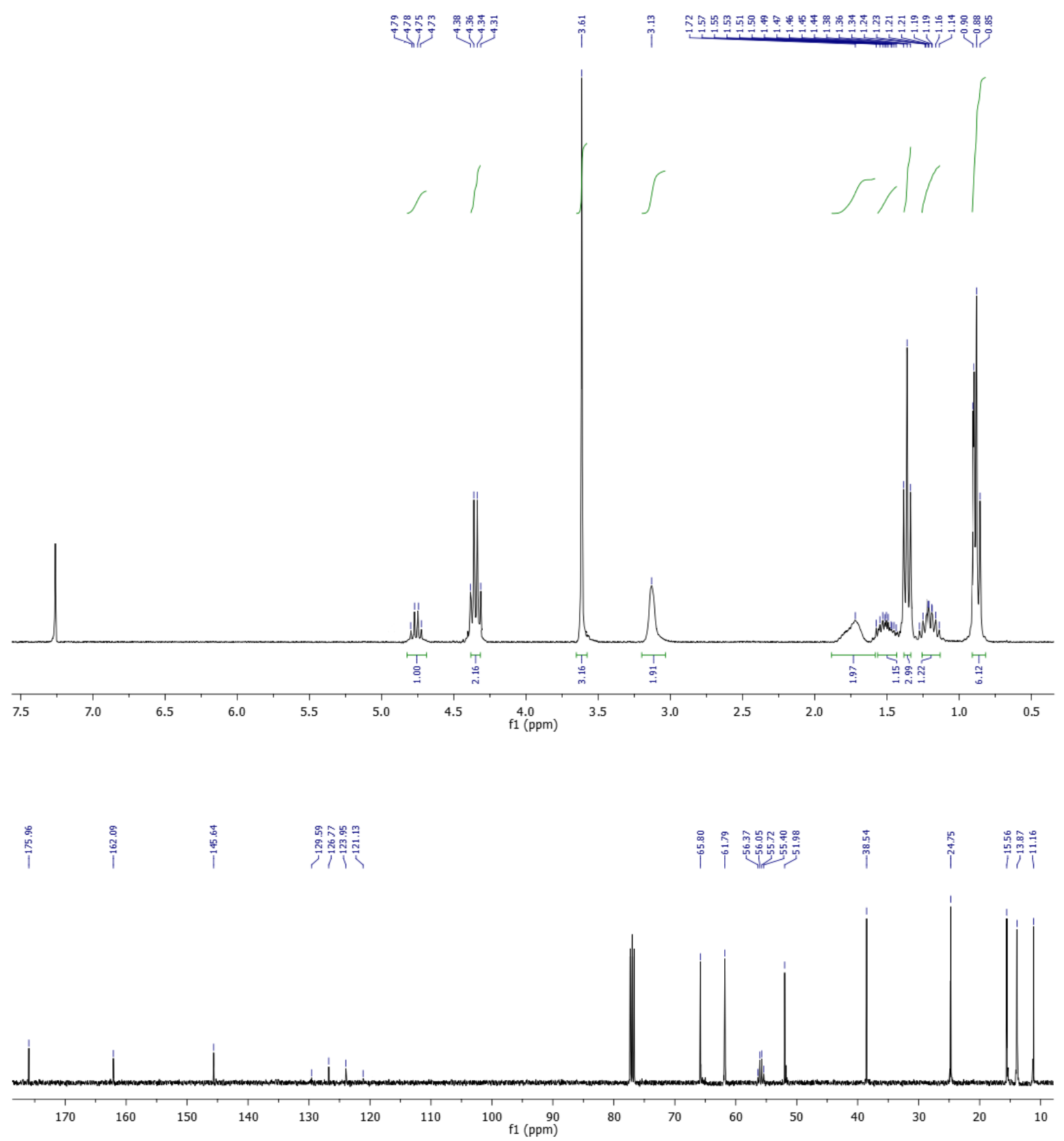


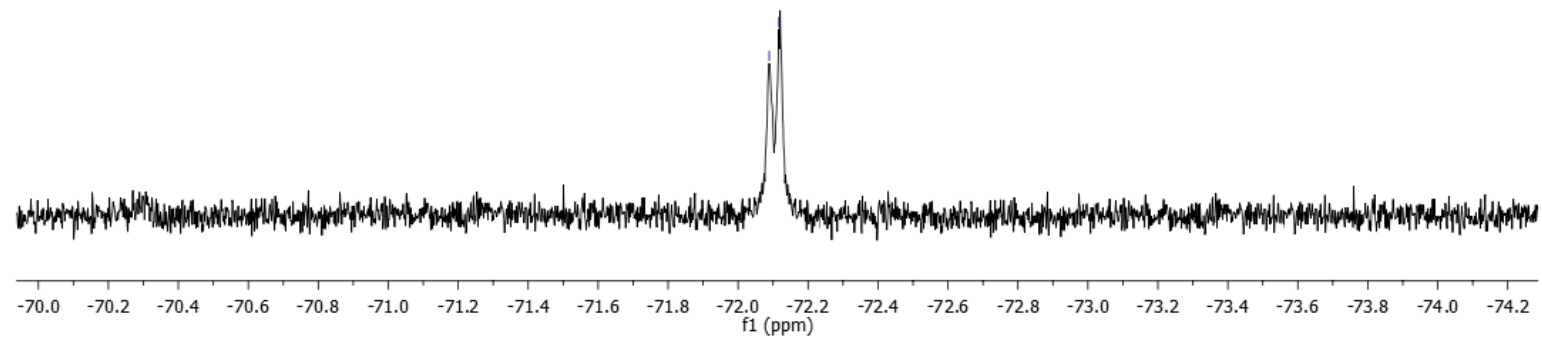


Methyl (2S,3S)-2-\{[(2S)-4-ethoxy-1,1,1-trifluoro-3-imino-4-oxobutan-2-yl]amino\}-3methylpentanoate<smiles>CCOC(=O)C(=N)C(NC(C(=O)OCC)C(F)(F)F)C(=O)OCC</smiles>
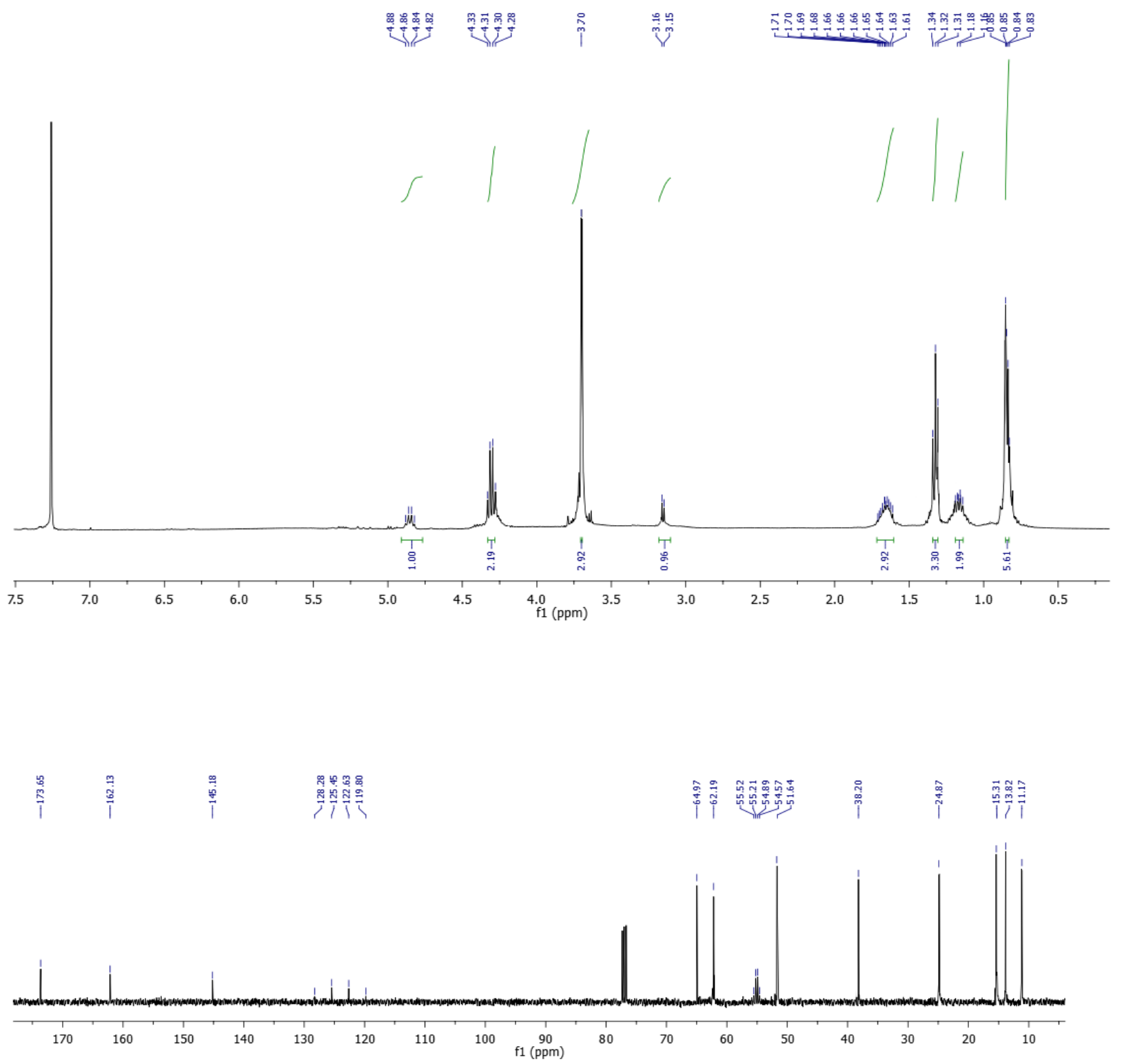


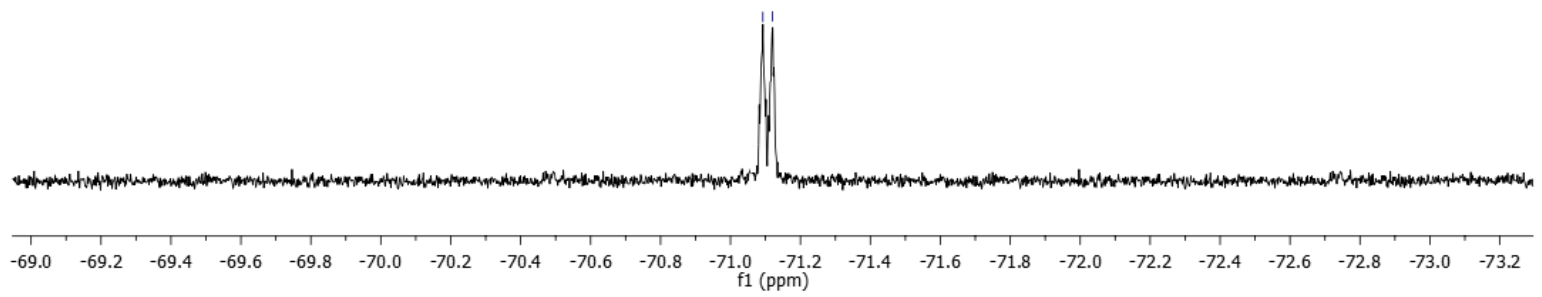


Methyl (2S)-2-\{[(2R)-4-ethoxy-1,1,1-trifluoro-3-imino-4-oxobutan-2-yl]amino\}-4methylpentanoate

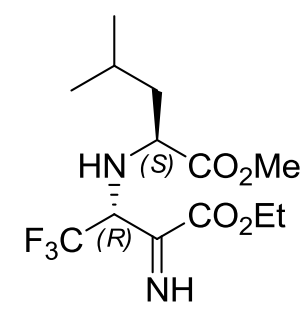

$17 \mathrm{c}$

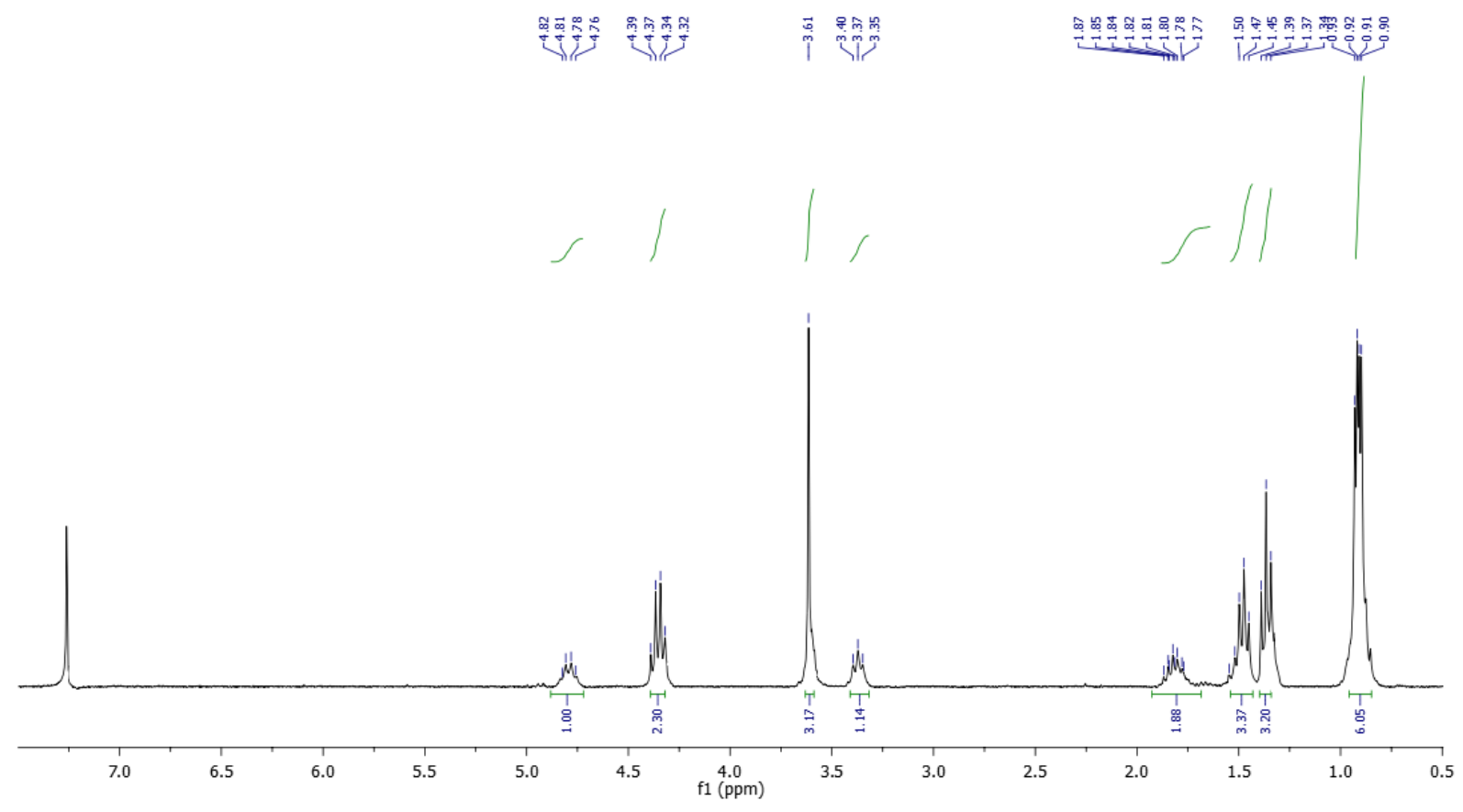

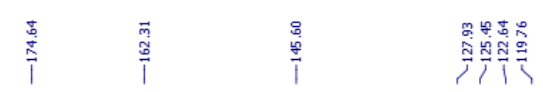
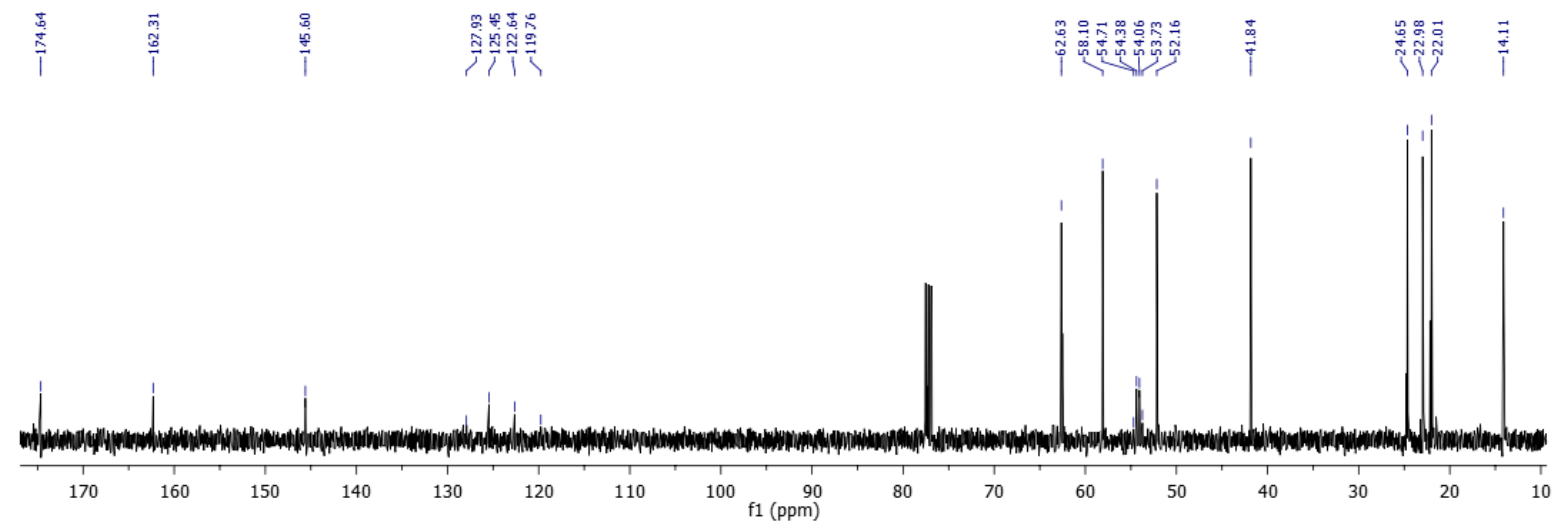


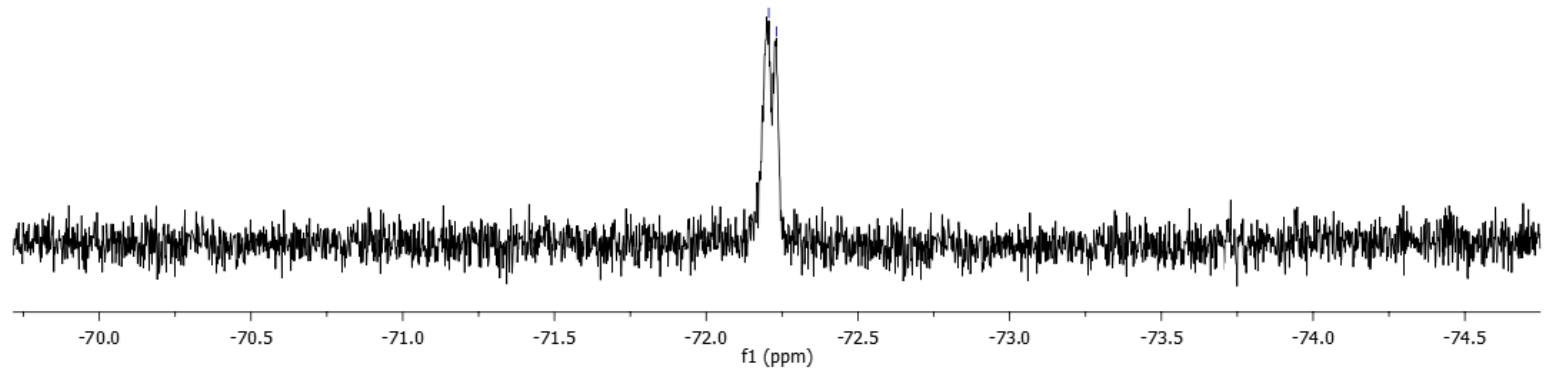


Methyl (2S)-2-\{[(2S)-4-ethoxy-1,1,1-trifluoro-3-imino-4-oxobutan-2-yl]amino\}-4methylpentanoate<smiles>CCOC(=O)C(=N)C(NC(CC(C)C)C(F)(F)F)C(F)(F)F</smiles>

18c

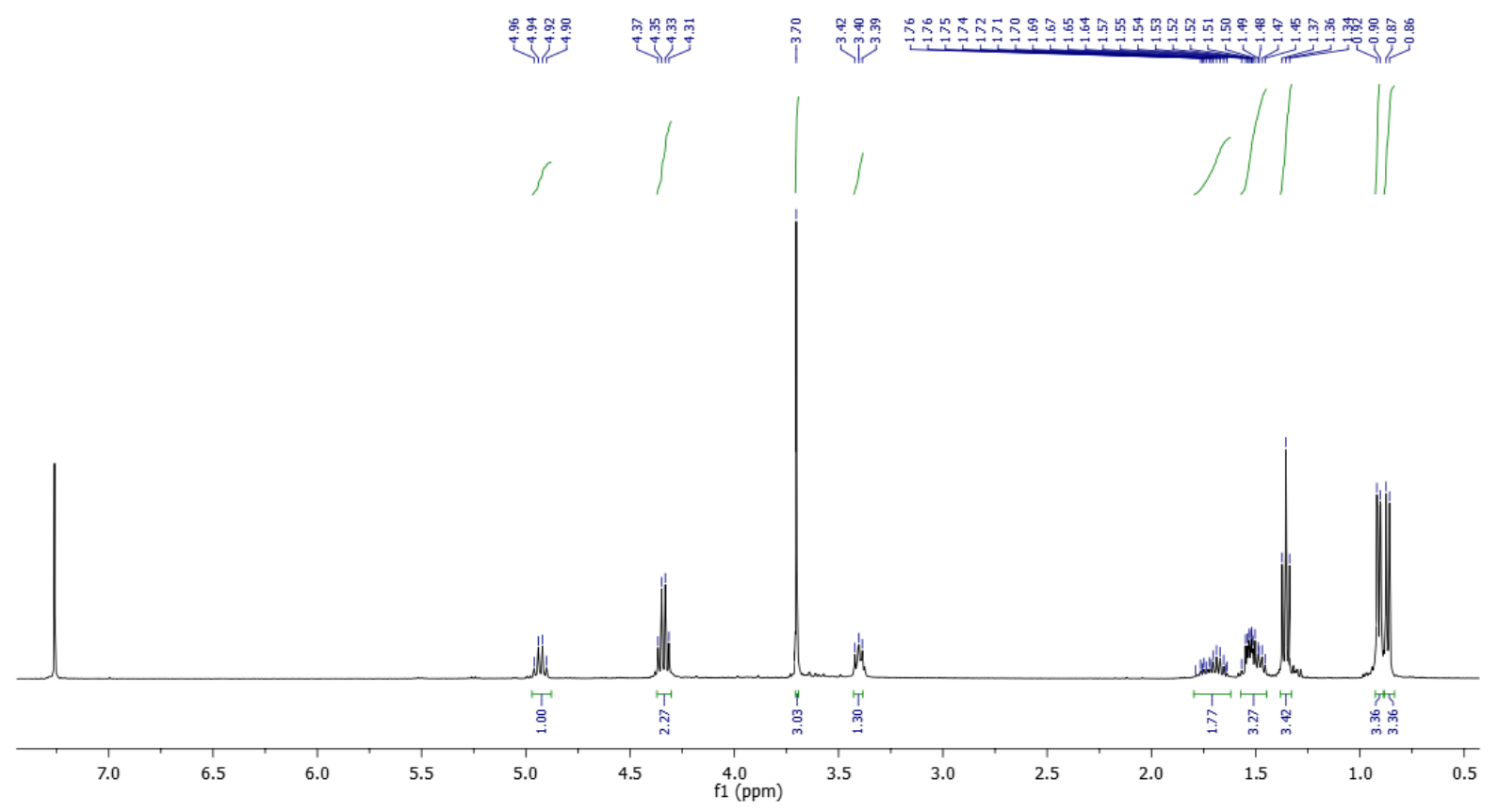

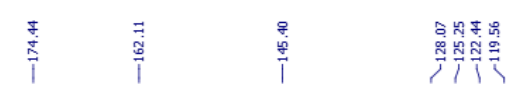
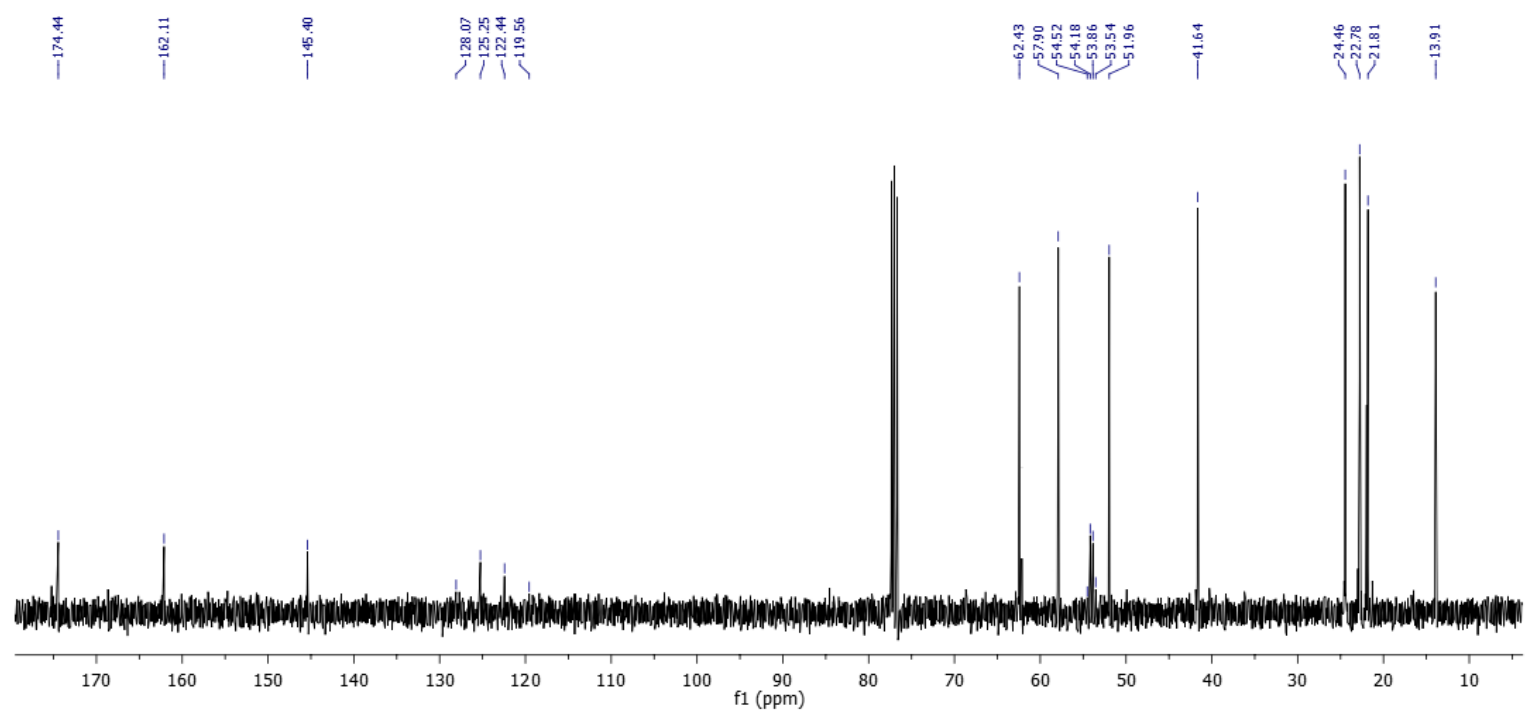


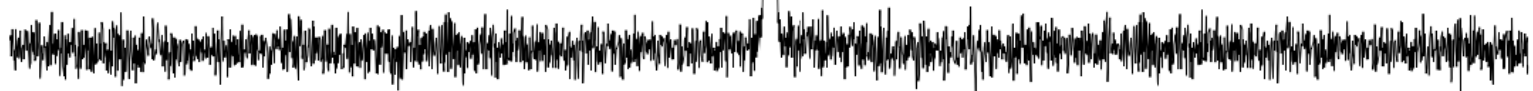

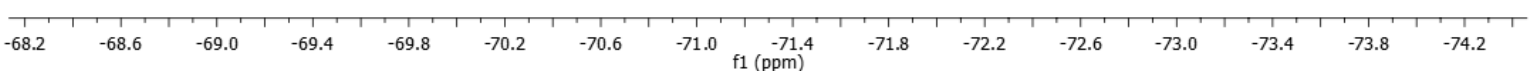




\section{D NMR spectra and optimized geometries to determine the absolute configuration of the new chiral centers.}

To determine the absolute configuration of the new trifluoromethyl substituted chiral center $\left(\mathrm{C}_{\beta}\right), 2 \mathrm{D}$ NMR spectra and optimized geometries of new optically pure $\beta$-amino carbonyl compounds obtained.

\section{D NMR spectra and optimized geometries}
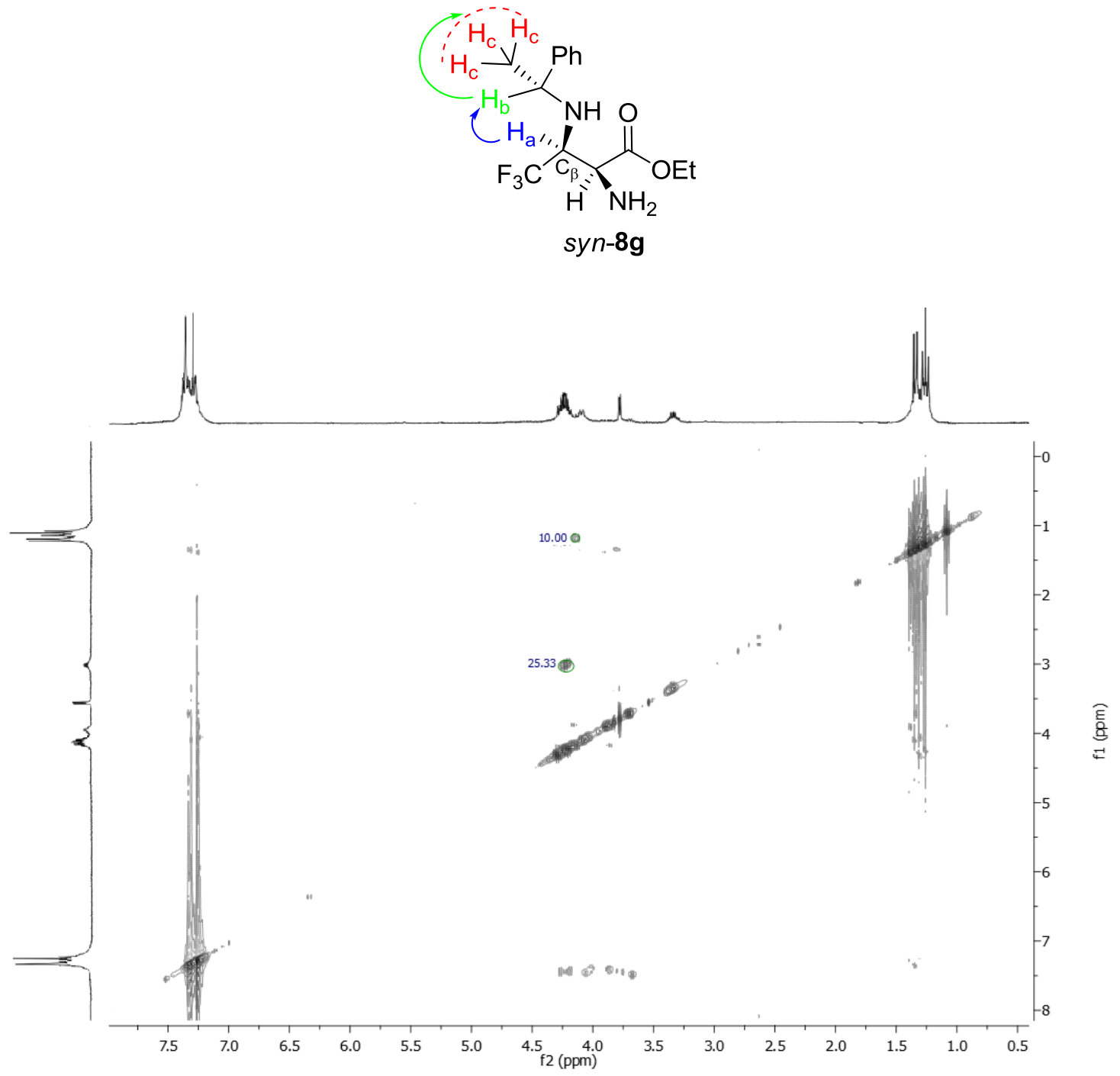

NOESY spectrum of syn-8g. The cross peak between the protons $\mathrm{H}_{\mathrm{b}}$ and $\mathrm{H}_{\mathrm{c}}$ was used as a distance ruler and its volume was set to 10.00 a.u.; the cross peak between $\mathrm{H}_{\mathrm{b}}$ and $\mathrm{H}_{\mathrm{a}}$ was measured $25.33 \mathrm{a}$. u. (corresponding to an interproton distance of $2.27 \AA$ ), in order to determine the absolute configuration. 


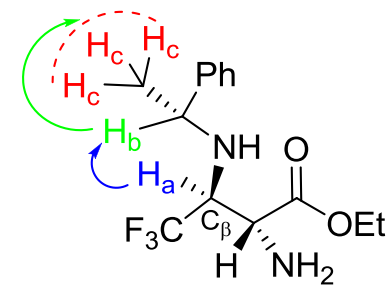

anti-8'g

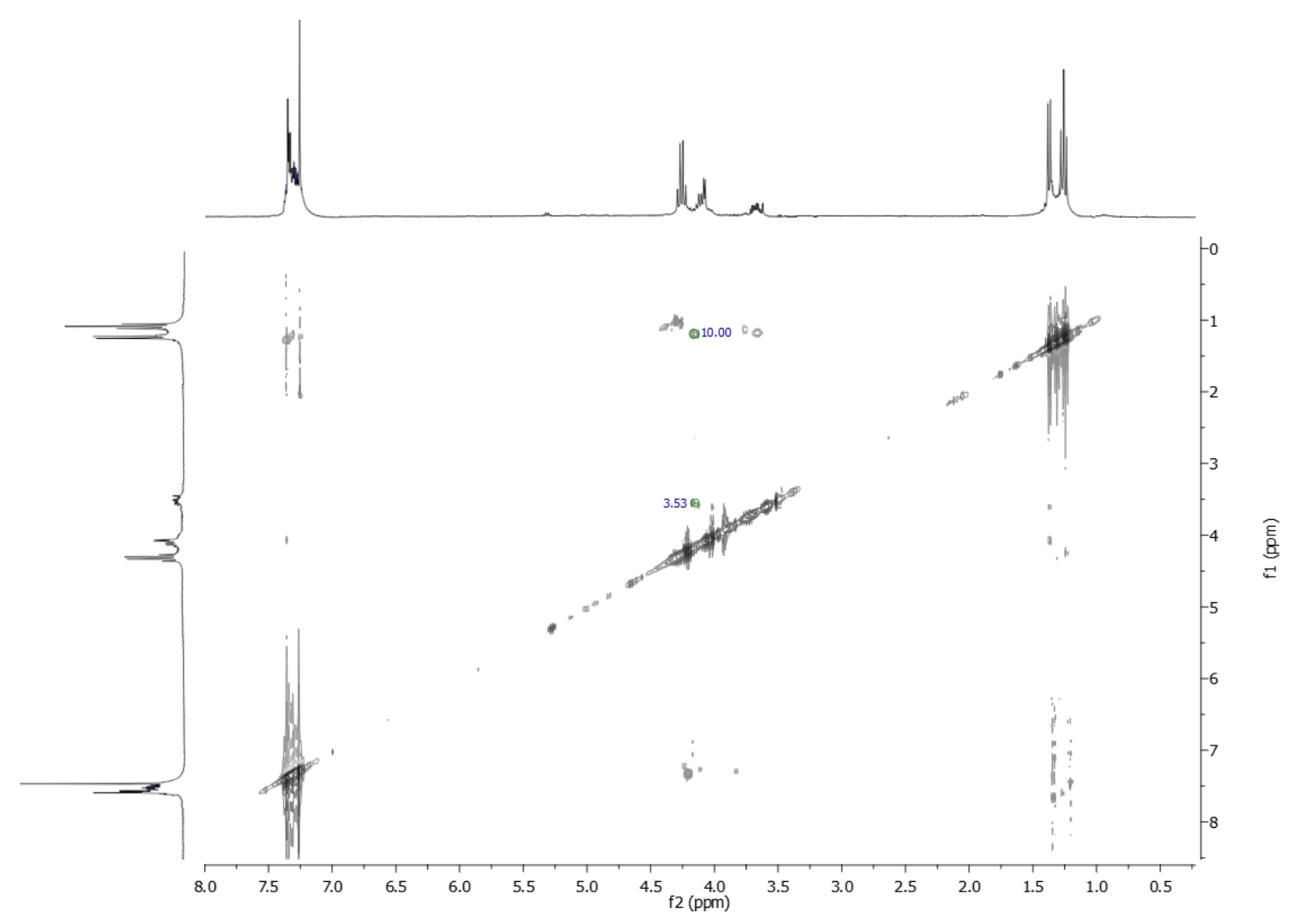

NOESY spectrum of anti-8'g. The cross peak between the protons $\mathrm{H}_{b}$ and $\mathrm{H}_{\mathrm{c}}$ was used as a distance ruler and its volume was set to 10.00 a.u.; the cross peak between $\mathrm{H}_{\mathrm{b}}$ and $\mathrm{H}_{\mathrm{a}}$ was measured $3.53 \mathrm{a}$. u. (corresponding to an interproton distance of $3.16 \AA$ ), in order to determine the absolute configuration.

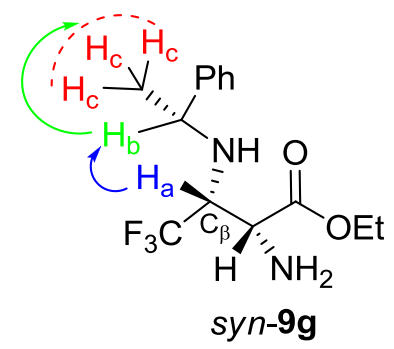




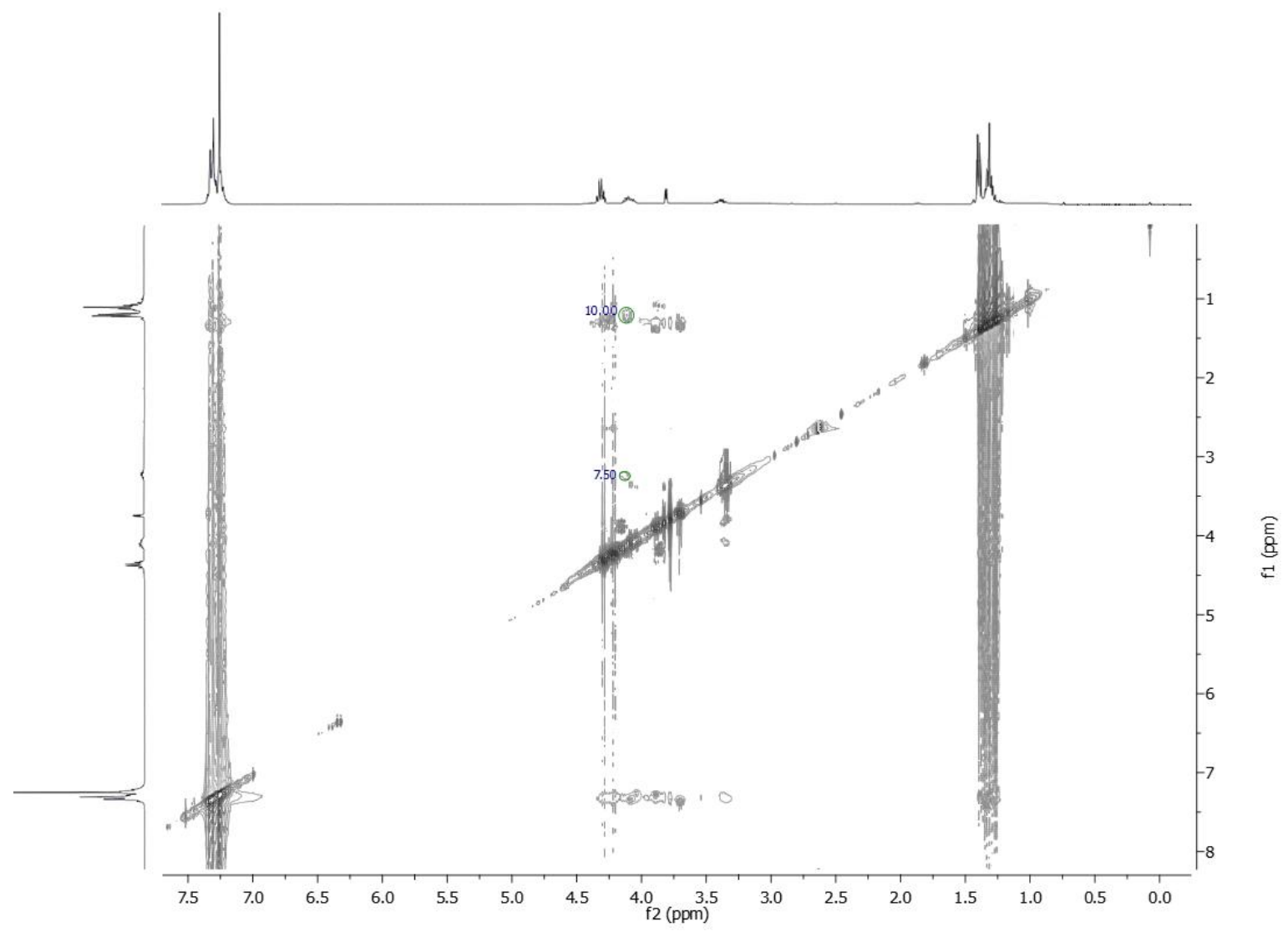

NOESY spectrum of syn-9g. The cross peak between the protons $\mathrm{H}_{\mathrm{b}}$ and $\mathrm{H}_{\mathrm{c}}$ was used as a distance ruler and its volume was set to 10.00 a.u.; the cross peak between $\mathrm{H}_{\mathrm{b}}$ and $\mathrm{H}_{\mathrm{a}}$ was measured $7.50 \mathrm{a}$. u. (corresponding to an interproton distance of $2.81 \AA$ ), in order to determine the absolute configuration. 


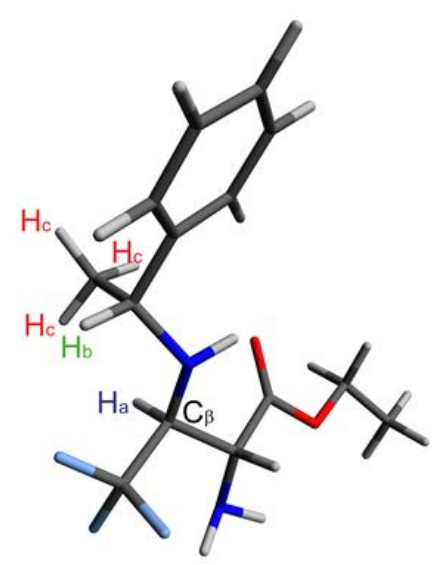

$\mathrm{H}_{\mathrm{a}} / \mathrm{H}_{\mathrm{b}}: 3.176 \AA$

anti-(R,S,R)-8'g

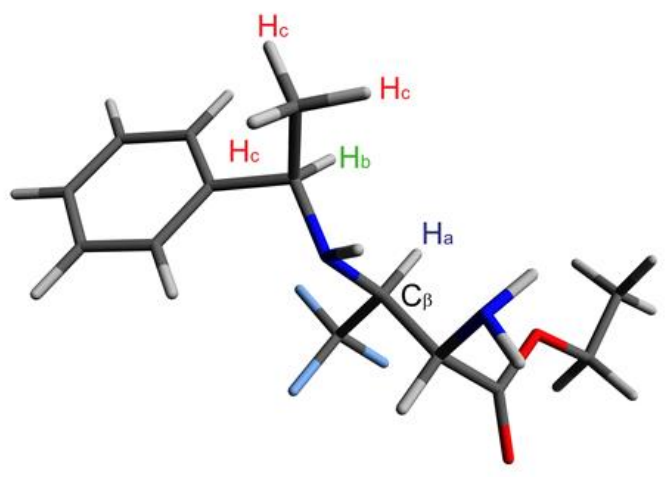

$\mathrm{H}_{\mathrm{a}} / \mathrm{H}_{\mathrm{b}}: 2.270 \AA$

syn-(R,S,S)-8g

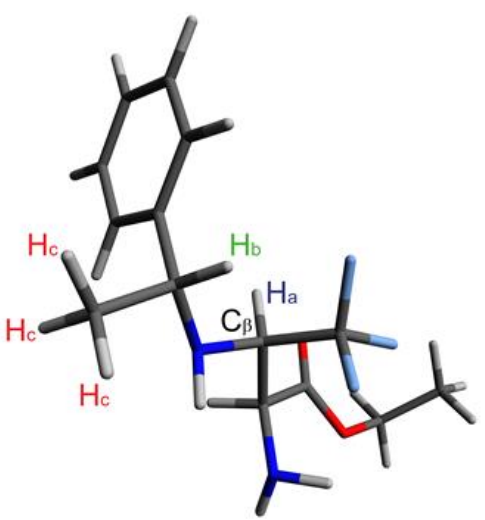

$\mathrm{H}_{\mathrm{a}} / \mathrm{H}_{\mathrm{b}}: 3.134 \AA$

anti-(R,R,S)-9'g

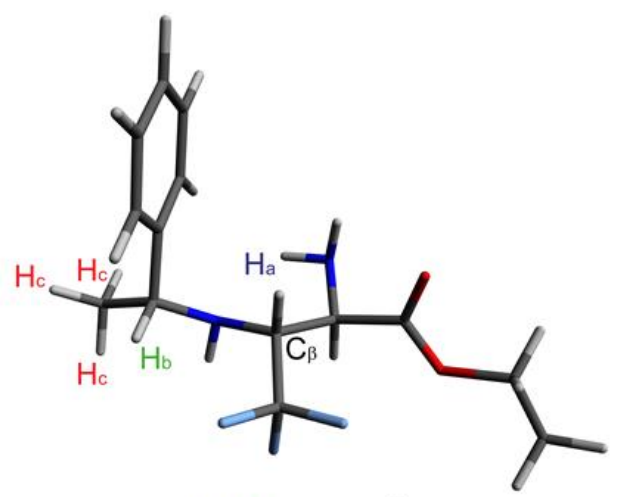

$\mathrm{H}_{\mathrm{a}} / \mathrm{H}_{\mathrm{b}}: 2.824 \AA$ syn-(R,R,R)-9g

Figure 1. Optimized geometries of all diastereomers. By NOESY analysis coupled with computational studies absolute configurations can be assigned to $\mathrm{C}_{\beta}$.

Calculated Coordinates:

\begin{tabular}{|c|c|c|c|c|c|}
\hline $\begin{array}{l}\text { Center } \\
\text { Number }\end{array}$ & $\begin{array}{l}\text { Atomic } \\
\text { Number }\end{array}$ & $\begin{array}{c}\text { Atomic } \\
\text { Type }\end{array}$ & $x^{\mathrm{CoO}}$ & inates ${ }_{Y}$ (Ans & ${ }_{\text {troms) }}{ }_{Z}$ \\
\hline $\begin{array}{r}1 \\
2 \\
3 \\
4 \\
5 \\
6 \\
7 \\
8 \\
9 \\
10 \\
11 \\
12 \\
13 \\
14 \\
15 \\
16 \\
17 \\
18 \\
19 \\
20 \\
21 \\
22 \\
23 \\
24\end{array}$ & $\begin{array}{l}7 \\
6 \\
6 \\
6 \\
1 \\
7 \\
6 \\
1 \\
6 \\
6 \\
1 \\
1 \\
1 \\
9 \\
9 \\
9 \\
8 \\
8 \\
6 \\
1 \\
6 \\
1 \\
1 \\
1\end{array}$ & $\begin{array}{l}0 \\
0 \\
0 \\
0 \\
0 \\
0 \\
0 \\
0 \\
0 \\
0 \\
0 \\
0 \\
0 \\
0 \\
0 \\
0 \\
0 \\
0 \\
0 \\
0 \\
0 \\
0 \\
0 \\
0\end{array}$ & $\begin{array}{r}3.169051 \\
1.902294 \\
0.688831 \\
2.045118 \\
1.668450 \\
-0.543650 \\
0.640331 \\
0.846335 \\
-1.767446 \\
-1.547443 \\
-1.050984 \\
-2.510314 \\
-0.938908 \\
0.758517 \\
1.631761 \\
-0.519023 \\
3.164649 \\
1.260611 \\
3.437602 \\
3.491110 \\
4.740378 \\
2.597998 \\
5.564338 \\
4.668078\end{array}$ & $\begin{array}{r}1.167568 \\
0.467973 \\
1.197234 \\
-0.952104 \\
0.342392 \\
0.530358 \\
2.658373 \\
1.230500 \\
0.686946 \\
0.563367 \\
-0.376728 \\
0.599499 \\
1.387075 \\
2.769718 \\
3.403535 \\
3.259921 \\
-1.525020 \\
-1.511088 \\
-2.884029 \\
-2.883722 \\
-3.318458 \\
-3.517655 \\
-2.668363 \\
-3.300253\end{array}$ & $\begin{array}{r}-0.140147 \\
-0.389311 \\
0.246467 \\
0.179834 \\
-1.460901 \\
-0.174527 \\
-0.220281 \\
1.333210 \\
0.658068 \\
2.179208 \\
2.437076 \\
2.697587 \\
2.564210 \\
-1.563149 \\
0.325587 \\
0.129840 \\
-0.281464 \\
0.922778 \\
0.164338 \\
1.256222 \\
-0.474508 \\
-0.134229 \\
-0.167483 \\
-1.565477\end{array}$ \\
\hline
\end{tabular}




$\begin{array}{rrrrrr}25 & 1 & 0 & 4.975123 & -4.340417 & -0.162876 \\ 26 & 1 & 0 & -0.345737 & -0.463204 & -0.217130 \\ 27 & 1 & 0 & 3.921784 & 0.600021 & -0.519624 \\ 28 & 1 & 0 & 3.183376 & 2.046633 & -0.648469 \\ 29 & 6 & 0 & -2.792028 & -0.326229 & 0.162620 \\ 30 & 6 & 0 & -2.581287 & -1.704845 & 0.319878 \\ 31 & 1 & 0 & -1.675028 & -2.063950 & 0.801337 \\ 32 & 6 & 0 & -3.523923 & -2.627760 & -0.134722 \\ 33 & 1 & 0 & -3.342888 & -3.691025 & -0.005031 \\ 34 & 6 & 0 & -4.698669 & -2.187706 & -0.749260 \\ 35 & 1 & 0 & -5.434014 & -2.905892 & -1.100076 \\ 36 & 6 & 0 & -4.920341 & -0.819174 & -0.908995 \\ 37 & 1 & 0 & -5.829749 & -0.466092 & -1.386827 \\ 38 & 6 & 0 & -3.971495 & 0.101194 & -0.458142 \\ 39 & 1 & 0 & -4.148281 & 1.165525 & -0.590339 \\ 40 & 1 & 0 & -2.173407 & 1.681787 & 0.459364 \\ -\end{array}$

\section{$(R, R, S)-9 ' \mathbf{g}$}

\begin{tabular}{|c|c|c|c|c|c|}
\hline \multirow{2}{*}{$\begin{array}{l}\text { Center } \\
\text { Number }\end{array}$} & \multirow{2}{*}{$\begin{array}{l}\text { Atomic } \\
\text { Number }\end{array}$} & \multirow{2}{*}{$\begin{array}{c}\text { Atomic } \\
\text { Type }\end{array}$} & \multicolumn{3}{|c|}{ Coordinates (Angstroms) } \\
\hline & & & $x$ & $\mathrm{Y}$ & Z \\
\hline 1 & 7 & 0 & -0.837184 & -1.210841 & -0.902088 \\
\hline 2 & 6 & 0 & -2.136484 & -1.360880 & -0.227680 \\
\hline 3 & 6 & 0 & 0.203797 & -0.435095 & -0.254221 \\
\hline 4 & 6 & 0 & 1.404734 & -0.330762 & -1.230529 \\
\hline 5 & 6 & 0 & 0.628299 & -0.994982 & 1.116844 \\
\hline 6 & 1 & 0 & -0.158096 & 0.578554 & -0.061823 \\
\hline 7 & 7 & 0 & 1.977887 & -1.643299 & -1.502966 \\
\hline 8 & 6 & 0 & 2.362424 & 0.790584 & -0.792954 \\
\hline 9 & 1 & 0 & 0.955128 & 0.033598 & -2.160611 \\
\hline 10 & 6 & 0 & -2.836305 & -0.014687 & -0.086991 \\
\hline 11 & 6 & 0 & -3.454889 & 0.338328 & 1.117788 \\
\hline 12 & 1 & 0 & -3.394341 & -0.338186 & 1.966719 \\
\hline 13 & 6 & 0 & -4.142025 & 1.548057 & 1.242229 \\
\hline 14 & 1 & 0 & -4.615167 & 1.805666 & 2.185557 \\
\hline 15 & 6 & 0 & -4.210756 & 2.427282 & 0.160651 \\
\hline 16 & 1 & 0 & -4.739642 & 3.371152 & 0.256140 \\
\hline 17 & 6 & 0 & -3.588225 & 2.088958 & -1.043922 \\
\hline 18 & 1 & 0 & -3.632322 & 2.770842 & -1.888586 \\
\hline 19 & 6 & 0 & -2.907713 & 0.877373 & -1.166369 \\
\hline 20 & 1 & 0 & -2.415260 & 0.617381 & -2.098528 \\
\hline 21 & 1 & 0 & -2.034125 & -1.785014 & 0.782681 \\
\hline 22 & 6 & 0 & -2.982385 & -2.343914 & -1.051719 \\
\hline 23 & 1 & 0 & 2.571303 & -1.934896 & -0.730201 \\
\hline 24 & 1 & 0 & 2.586334 & -1.586246 & -2.314713 \\
\hline 25 & 1 & 0 & -3.131623 & -1.967174 & -2.067861 \\
\hline 26 & 1 & 0 & -3.961107 & -2.487025 & -0.586237 \\
\hline 27 & 1 & 0 & -2.489600 & -3.320989 & -1.113578 \\
\hline 28 & 1 & 0 & -0.451595 & -2.116934 & -1.156240 \\
\hline 29 & 8 & 0 & 1.981693 & 1.909759 & -0.509548 \\
\hline 30 & 8 & 0 & 3.646565 & 0.401305 & -0.805911 \\
\hline 31 & 6 & 0 & 4.635637 & 1.384370 & -0.390327 \\
\hline 32 & 6 & 0 & 4.809598 & 1.371218 & 1.119132 \\
\hline 33 & 1 & 0 & 3.885869 & 1.672288 & 1.618808 \\
\hline 34 & 1 & 0 & 5.599989 & 2.073020 & 1.403338 \\
\hline 35 & 1 & 0 & 5.091528 & 0.374727 & 1.470569 \\
\hline 36 & 1 & 0 & 4.323547 & 2.366472 & -0.750696 \\
\hline 37 & 1 & 0 & 5.549384 & 1.081350 & -0.904983 \\
\hline 38 & 9 & 0 & 0.787731 & -2.338829 & 1.093332 \\
\hline 39 & 9 & 0 & -0.289976 & -0.723218 & 2.071911 \\
\hline 40 & 9 & 0 & 1.806088 & -0.470533 & 1.550059 \\
\hline
\end{tabular}

\section{$(R, S, S)-\mathbf{8 g}$}

\begin{tabular}{|c|c|c|c|c|c|}
\hline Center & Atomic & Atomic & Coor & inates (An & troms) \\
\hline Number & Number & Type & $\mathrm{x}$ & $\mathrm{Y}$ & Z \\
\hline $\begin{array}{r}1 \\
2 \\
3 \\
4 \\
5 \\
6 \\
7 \\
8 \\
9 \\
10 \\
11 \\
12 \\
13 \\
14 \\
15 \\
16\end{array}$ & $\begin{array}{l}7 \\
6 \\
6 \\
6 \\
1 \\
6 \\
7 \\
6 \\
1 \\
6 \\
6 \\
1 \\
6 \\
1 \\
6 \\
1\end{array}$ & $\begin{array}{l}0 \\
0 \\
0 \\
0 \\
0 \\
0 \\
0 \\
0 \\
0 \\
0 \\
0 \\
0 \\
0 \\
0 \\
0 \\
0\end{array}$ & $\begin{array}{r}0.571251 \\
1.575902 \\
-0.587440 \\
-1.752436 \\
-0.924361 \\
-0.271361 \\
-1.909867 \\
-3.073259 \\
-1.476626 \\
2.845386 \\
3.672180 \\
3.373215 \\
4.870255 \\
5.495590 \\
5.260118 \\
6.190407\end{array}$ & $\begin{array}{r}0.912673 \\
1.411562 \\
0.222668 \\
0.347873 \\
0.628433 \\
-1.249742 \\
1.759939 \\
-0.249940 \\
-0.207317 \\
0.560022 \\
0.591617 \\
1.177832 \\
-0.123044 \\
-0.090146 \\
-0.884481 \\
-1.444810\end{array}$ & $\begin{array}{r}-0.757305 \\
0.196647 \\
-0.219833 \\
-1.243216 \\
0.749747 \\
0.046507 \\
-1.613497 \\
-0.750005 \\
-2.142328 \\
0.230282 \\
1.361076 \\
2.227759 \\
1.392963 \\
2.280897 \\
0.288601 \\
0.311460\end{array}$ \\
\hline
\end{tabular}




$\begin{array}{rrrrrr}17 & 6 & 0 & 4.439711 & -0.926456 & -0.840341 \\ 18 & 1 & 0 & 4.730321 & -1.522964 & -1.700946 \\ 19 & 6 & 0 & 3.241570 & -0.209745 & -0.868425 \\ 20 & 1 & 0 & 2.587654 & -0.256866 & -1.732605 \\ 21 & 1 & 0 & 1.153482 & 1.415935 & 1.214225 \\ 22 & 6 & 0 & 1.933437 & 2.864538 & -0.166219 \\ 23 & 1 & 0 & -2.400843 & 2.259575 & -0.874810 \\ 24 & 1 & 0 & -2.472284 & 1.838918 & -2.456332 \\ 25 & 1 & 0 & 2.327895 & 2.912374 & -1.187063 \\ 26 & 1 & 0 & 2.698496 & 3.258141 & 0.507596 \\ 27 & 1 & 0 & 1.050350 & 3.509714 & -0.103017 \\ 28 & 1 & 0 & 0.200837 & 1.680504 & -1.313477 \\ 29 & 8 & 0 & -3.724899 & -1.073584 & -1.357531 \\ 30 & 8 & 0 & -3.465349 & 0.324912 & 0.402832 \\ 31 & 6 & 0 & -4.742366 & -0.110990 & 0.942258 \\ 32 & 6 & 0 & -4.986297 & 0.661862 & 2.222255 \\ 33 & 1 & 0 & -4.197486 & 0.467574 & 2.954315 \\ 34 & 1 & 0 & -5.941780 & 0.355108 & 2.657703 \\ 35 & 1 & 0 & -5.026681 & 1.737896 & 2.030319 \\ 36 & 1 & 0 & -4.692287 & -1.189697 & 1.114819 \\ 37 & 1 & 0 & -5.517591 & 0.074918 & 0.193440 \\ 38 & 9 & 0 & 0.109048 & -1.907192 & -1.066730 \\ 39 & 9 & 0 & -1.356541 & -1.899791 & 0.545278 \\ 40 & 9 & 0 & 0.712115 & -1.388405 & 0.959917 \\ ------------------------------15\end{array}$

\section{$(R, R, R)-9 \mathbf{g}$}

\begin{tabular}{|c|c|c|c|c|c|}
\hline \multirow[t]{2}{*}{$\begin{array}{l}\text { Center } \\
\text { Number }\end{array}$} & \multirow[t]{2}{*}{$\begin{array}{l}\text { Atomic } \\
\text { Number }\end{array}$} & $\begin{array}{c}\text { Atomic } \\
\text { Type }\end{array}$ & \multicolumn{3}{|c|}{ Coordinates (Angstroms) } \\
\hline & & & & & \\
\hline 1 & 7 & 0 & 0.839786 & 1.075260 & -1.03468 \\
\hline 2 & 6 & 0 & 2.153117 & 1.373304 & -0.42120 \\
\hline 3 & 6 & 0 & -0.202648 & 0.515810 & -0.18686 \\
\hline 4 & 6 & 0 & -1.198442 & -0.305153 & -1.078982 \\
\hline 5 & 6 & 0 & -0.926183 & 1.589373 & 0.627763 \\
\hline 6 & 1 & 0 & 0.253906 & -0.173849 & 0.52972 \\
\hline 7 & 6 & 0 & -2.191530 & -1.089460 & -0.22551 \\
\hline 8 & 7 & 0 & -0.519110 & -1.276454 & -1.93240 \\
\hline 9 & 6 & 0 & 2.850400 & 0.092993 & 0.02098 \\
\hline 10 & 6 & 0 & 3.298616 & -0.044914 & 1.33962 \\
\hline 11 & 1 & 0 & 3. 117971 & 0.757615 & 2.05073 \\
\hline 12 & 6 & 0 & 3.971538 & -1.198312 & 1.75203 \\
\hline 13 & 1 & 0 & 4.310228 & -1.287044 & 2.78011 \\
\hline 14 & 6 & 0 & 4.200742 & -2.233993 & 0.84633 \\
\hline 15 & 1 & 0 & 4.720894 & -3.133000 & 1.16363 \\
\hline 16 & 6 & 0 & 3.753822 & -2.109595 & -0.47261 \\
\hline 17 & 1 & 0 & 3.928261 & -2.912306 & -1.18363 \\
\hline 18 & 6 & 0 & 3.084413 & -0.956294 & -0.88036 \\
\hline 19 & 1 & 0 & 2.740607 & -0.865913 & -1.90631 \\
\hline 20 & 1 & 0 & 2.043750 & 2.009456 & 0.46763 \\
\hline 21 & 6 & 0 & 2.988372 & 2.150089 & -1.44857 \\
\hline 22 & 1 & 0 & 3.130038 & 1.563201 & -2.36089 \\
\hline 23 & 1 & 0 & 3.970933 & 2.390445 & -1.03465 \\
\hline 24 & 1 & 0 & 2.494608 & 3.090917 & -1.71726 \\
\hline 25 & 1 & 0 & 0.489055 & 1.898826 & -1.51776 \\
\hline 26 & 9 & 0 & -1.492224 & 2.518618 & -0.18221 \\
\hline 27 & 9 & 0 & -0.089068 & 2.251189 & 1.45959 \\
\hline 28 & 9 & 0 & -1.914669 & 1.077056 & 1. 39901 \\
\hline 29 & 8 & 0 & -1.858579 & -2.007581 & 0.49959 \\
\hline 30 & 8 & 0 & -3.449964 & -0.666791 & -0.38695 \\
\hline 31 & 6 & 0 & -4.462760 & -1.340726 & 0.408591 \\
\hline 32 & 6 & 0 & -5.795373 & -0.693800 & 0.09079 \\
\hline 33 & 1 & 0 & -5.783742 & 0.369993 & 0.342757 \\
\hline 34 & 1 & 0 & -6.585003 & -1.177007 & 0.673801 \\
\hline 35 & 1 & 0 & -6.038938 & -0.798155 & -0.969902 \\
\hline 36 & 1 & 0 & -4.194692 & -1.238401 & 1.463839 \\
\hline 37 & 1 & 0 & -4.449805 & -2.405160 & 0.158290 \\
\hline 38 & 1 & 0 & -0.130388 & -2.003966 & -1.333032 \\
\hline 39 & 1 & 0 & 0.278071 & -0.796425 & -2.348279 \\
\hline 40 & 1 & 0 & -1.753003 & 0.396394 & -1.709849 \\
\hline
\end{tabular}



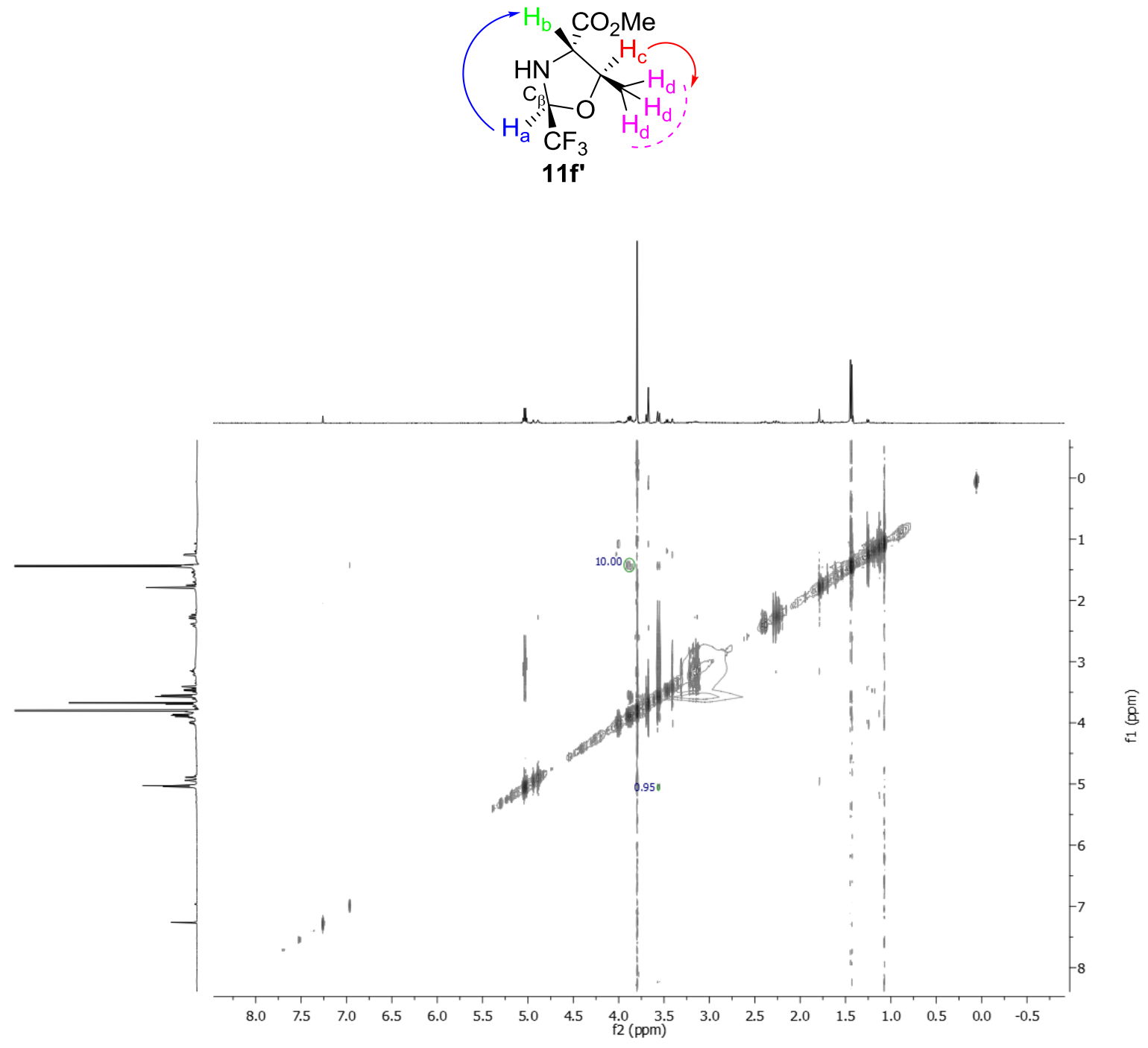

NOESY spectrum of 11f'. The cross peak between the protons $\mathrm{H}_{\mathrm{c}}$ and $\mathrm{H}_{\mathrm{d}}$ was used as a distance ruler and its volume was set to 10.00 a.u.; the cross peak between $\mathrm{H}_{\mathrm{b}}$ and $\mathrm{H}_{\mathrm{a}}$ was measured 0.95 a.u. (corresponding to an interproton distance of $3.97 \AA$ ), in order to determine the absolute configuration.

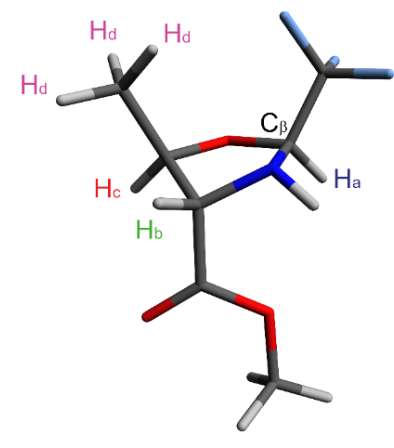

$\mathrm{H}_{\mathrm{a}} / \mathrm{H}_{\mathrm{b}}: 3.982 \AA$

$(S, R, S)-11 f^{\prime}$

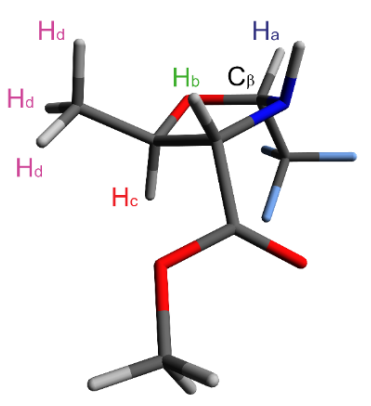

$\mathrm{H}_{\mathrm{a}} / \mathrm{H}_{\mathrm{b}}: 3.810 \AA$

$(S, R, R)$

diastereomer obtained

Figure 1. Optimized geometries of all diastereomers. By NOESY analysis coupled with computational studies absolute configurations can be assigned to $\mathrm{C}_{\beta}$. 
Calculated Coordinates:

$(S, R, S)-11 \mathbf{f}^{\prime}$

\begin{tabular}{|c|c|c|c|c|c|}
\hline Center & Atomic & Atomic & Coor & inates (An & troms) \\
\hline Number & Number & Type & $\mathrm{x}$ & $\mathrm{Y}$ & Z \\
\hline $\begin{array}{r}1 \\
2 \\
3 \\
4 \\
5 \\
6 \\
7 \\
8 \\
9 \\
10 \\
11 \\
12 \\
13 \\
14 \\
15 \\
16 \\
17 \\
18 \\
19 \\
20 \\
21 \\
22 \\
23 \\
24\end{array}$ & $\begin{array}{l}6 \\
6 \\
7 \\
8 \\
6 \\
1 \\
6 \\
6 \\
1 \\
1 \\
1 \\
1 \\
1 \\
9 \\
9 \\
9 \\
6 \\
1 \\
8 \\
8 \\
6 \\
1 \\
1 \\
1\end{array}$ & $\begin{array}{l}0 \\
0 \\
0 \\
0 \\
0 \\
0 \\
0 \\
0 \\
0 \\
0 \\
0 \\
0 \\
0 \\
0 \\
0 \\
0 \\
0 \\
0 \\
0 \\
0 \\
0 \\
0 \\
0 \\
0\end{array}$ & $\begin{array}{r}0.495946 \\
-0.386177 \\
-0.270168 \\
-0.941965 \\
-1.478437 \\
0.225167 \\
-0.903733 \\
-2.327597 \\
-0.379286 \\
-2.049022 \\
-1.036707 \\
-2.163063 \\
0.317360 \\
-3.122704 \\
-2.910390 \\
-2.286696 \\
1.925385 \\
0.585048 \\
2.315931 \\
2.622000 \\
3.671487 \\
3.864450 \\
3.773393 \\
4.365731\end{array}$ & $\begin{array}{r}1.109598 \\
1.791770 \\
-0.098910 \\
0.664138 \\
2.684483 \\
2.347943 \\
-0.486298 \\
-0.996433 \\
-1.293642 \\
2.160487 \\
3.586636 \\
2.988582 \\
-0.841443 \\
-0.079420 \\
-1.372519 \\
-2.079824 \\
0.902144 \\
1.737176 \\
-0.379782 \\
1.812038 \\
-0.668197 \\
-1.715900 \\
-0.323647 \\
-0.092425\end{array}$ & $\begin{array}{r}-0.680078 \\
0.383559 \\
-0.967928 \\
1.102953 \\
-0.190254 \\
1.098081 \\
0.286819 \\
0.033378 \\
0.822195 \\
-0.959611 \\
-0.625350 \\
0.606125 \\
-1.331949 \\
-0.544688 \\
1.191029 \\
-0.776223 \\
-0.158685 \\
-1.573958 \\
-0.224916 \\
0.243357 \\
0.220554 \\
0.120580 \\
1.253091 \\
-0.397849\end{array}$ \\
\hline
\end{tabular}

$(S, R, R)$

\begin{tabular}{|c|c|c|c|c|c|}
\hline Center & Atomic & Atomic & Coor & inates (An & troms) \\
\hline Number & Number & Type & $\mathrm{x}$ & $\mathrm{Y}$ & Z \\
\hline $\begin{array}{r}1 \\
2 \\
3 \\
4 \\
5 \\
6 \\
7 \\
8 \\
9 \\
10 \\
11 \\
12 \\
13 \\
14 \\
15 \\
16 \\
17 \\
18 \\
19 \\
20 \\
21 \\
22 \\
23 \\
24\end{array}$ & $\begin{array}{l}6 \\
6 \\
8 \\
1 \\
6 \\
6 \\
7 \\
1 \\
6 \\
6 \\
1 \\
1 \\
1 \\
1 \\
1 \\
8 \\
8 \\
6 \\
1 \\
1 \\
1 \\
9 \\
9 \\
9\end{array}$ & $\begin{array}{l}0 \\
0 \\
0 \\
0 \\
0 \\
0 \\
0 \\
0 \\
0 \\
0 \\
0 \\
0 \\
0 \\
0 \\
0 \\
0 \\
0 \\
0 \\
0 \\
0 \\
0 \\
0 \\
0 \\
0\end{array}$ & $\begin{array}{r}0.929006 \\
0.444619 \\
-0.948735 \\
0.918116 \\
0.597179 \\
-1.389290 \\
-0.249112 \\
-1.806468 \\
-2.539587 \\
2.171113 \\
1.175461 \\
-0.261325 \\
0.199173 \\
0.052530 \\
1.651088 \\
3.214814 \\
2.237351 \\
4.472598 \\
4.394219 \\
5.191532 \\
4.768193 \\
-2.207250 \\
-2.930316 \\
-3.604654\end{array}$ & $\begin{array}{r}-0.081587 \\
1.329177 \\
1.291059 \\
2.097552 \\
1.664537 \\
-0.049754 \\
-0.879785 \\
-0.245405 \\
-0.268749 \\
-0.577960 \\
-0.061330 \\
-1.778554 \\
0.864452 \\
2.586107 \\
1.824957 \\
0.224023 \\
-1.558326 \\
-0.137888 \\
-0.101878 \\
0.598372 \\
-1.143089 \\
0.028718 \\
-1.563472 \\
0.489934\end{array}$ & $\begin{array}{r}-0.809144 \\
-0.396839 \\
-0.782309 \\
-1.012965 \\
1.081951 \\
-0.845770 \\
-0.498518 \\
-1.847675 \\
0.145023 \\
-0.083050 \\
-1.883540 \\
-0.967376 \\
1.709410 \\
1.303415 \\
1.327840 \\
-0.370869 \\
0.624219 \\
0.236728 \\
1.325294 \\
-0.119048 \\
-0.070573 \\
1.411873 \\
0.108895 \\
-0.189310\end{array}$ \\
\hline
\end{tabular}

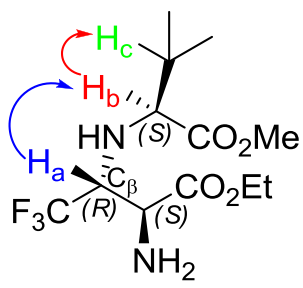

anti-15'a 


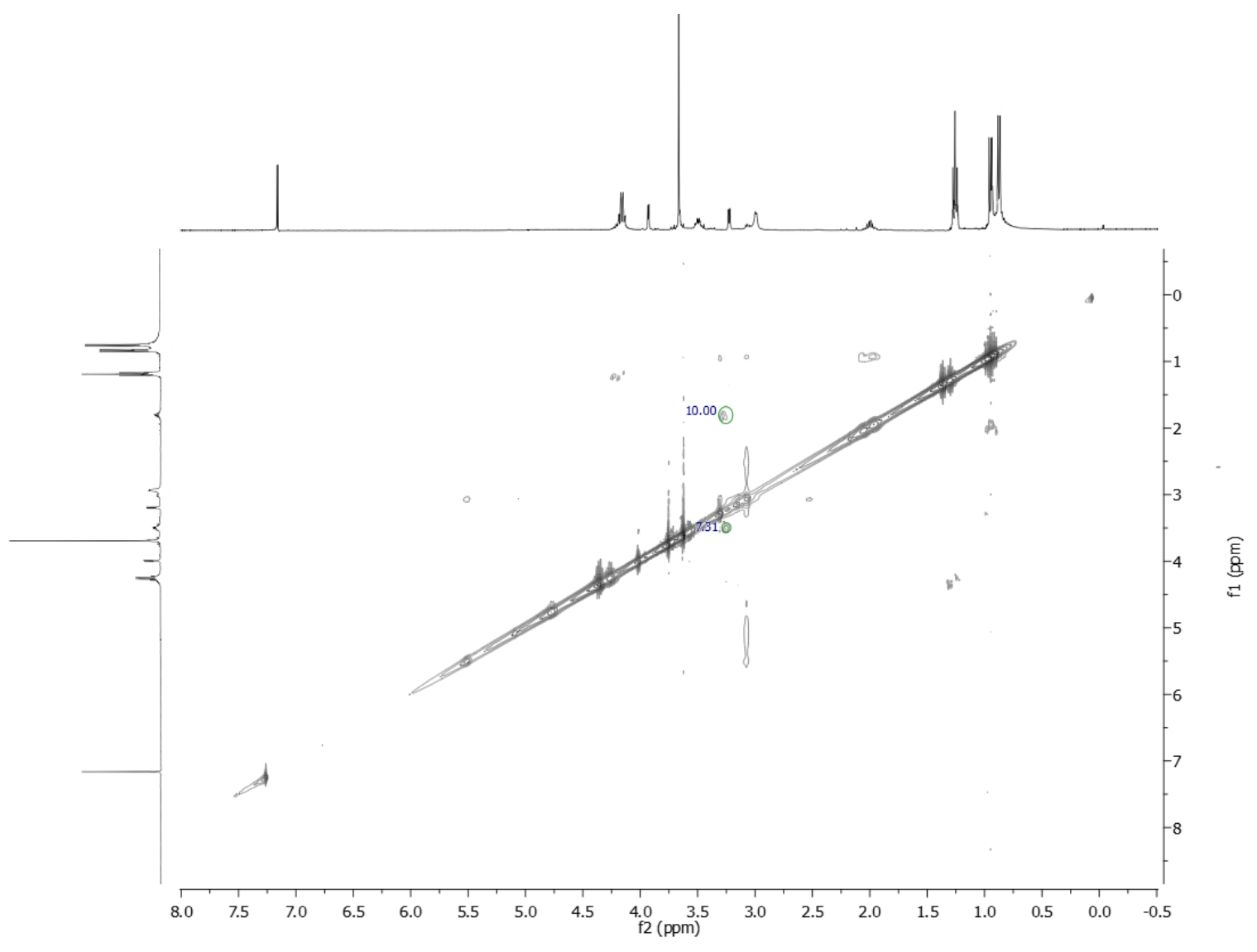

NOESY spectrum of 15'a. The cross peak between the protons $\mathrm{H}_{\mathrm{b}}$ and $\mathrm{H}_{\mathrm{c}}$ was used as a distance ruler and its volume was set to 10.00 a.u.; the cross peak between $H_{d}$ and $H_{e}$ was measured 7.31 a.u. (corresponding to an interproton distance of $3.19 \AA$ ), in order to determine the absolute configuration.

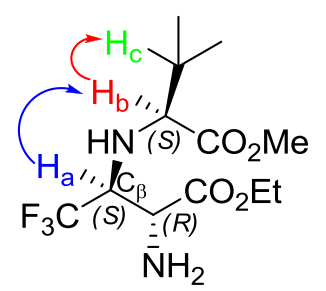

anti-16'a 


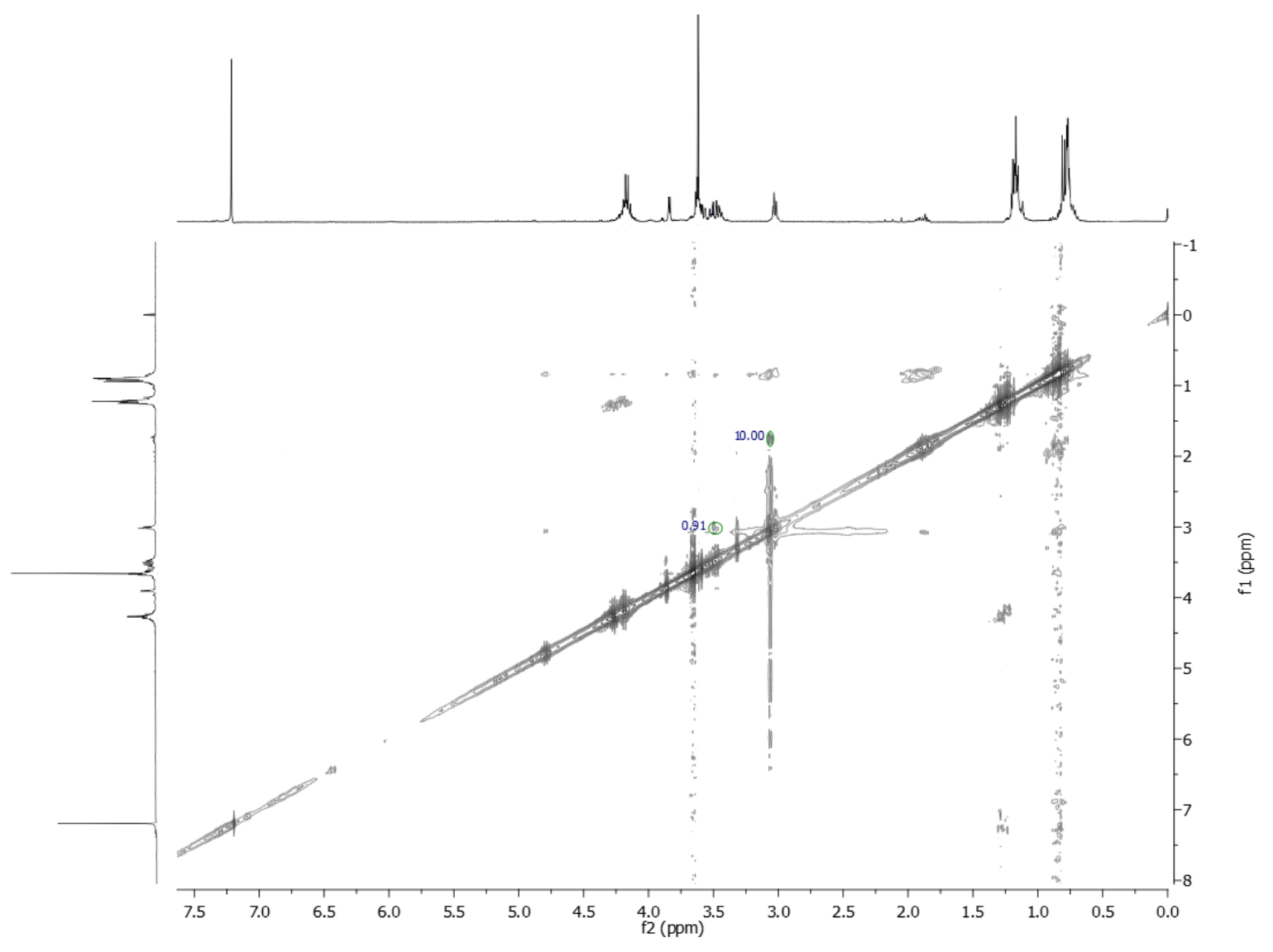

NOESY spectrum of 15'a. The cross peak between the protons $\mathrm{H}_{\mathrm{b}}$ and $\mathrm{H}_{\mathrm{c}}$ was used as a distance ruler and its volume was set to 10.00 a.u.; the cross peak between $\mathrm{H}_{\mathrm{a}}$ and $\mathrm{H}_{\mathrm{b}}$ was measured 0.91 a.u. (corresponding to an interproton distance of $3.77 \AA$ ), in order to determine the absolute configuration. 


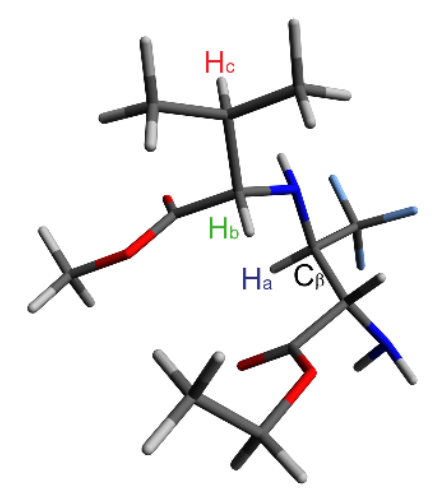

$\mathrm{H}_{\mathrm{a}} / \mathrm{H}_{\mathrm{b}}: 3.157 \AA$

anti-(S,R,S)-15'a

major diastereomer obtained

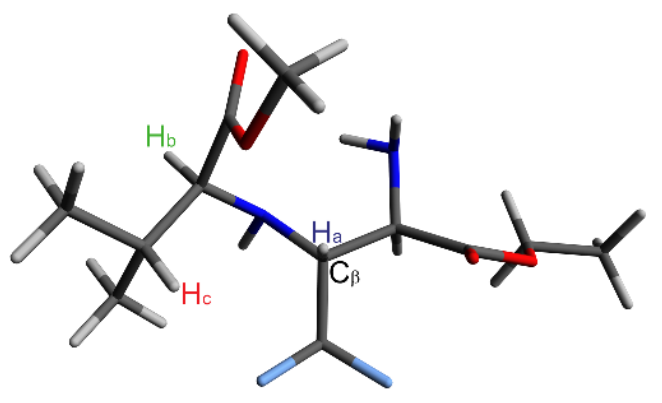

$\mathrm{H}_{\mathrm{a}} / \mathrm{H}_{\mathrm{b}}: 3.619 \AA$ $\operatorname{syn}-(S, R, R)$

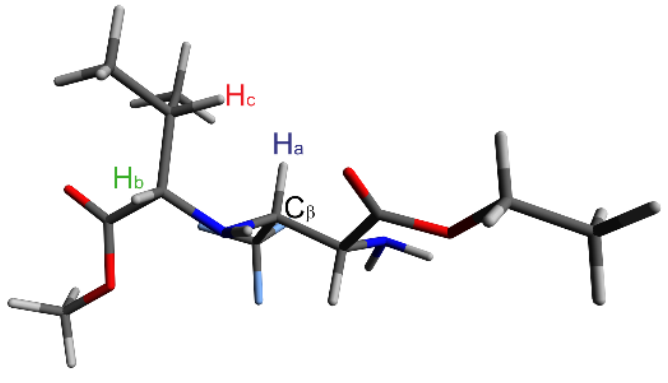

$\mathrm{H}_{\mathrm{a}} / \mathrm{H}_{\mathrm{b}}: 3.662 \AA$

anti-(S,S,R)-16'a

minor diastereomer obtained

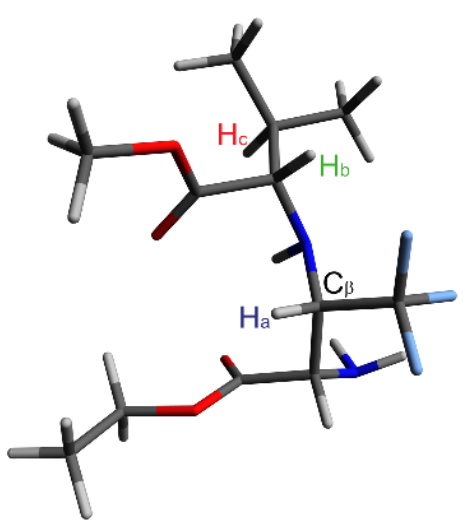

$\mathrm{H}_{a} / \mathrm{H}_{b}: 2.834 \AA$

syn- $(S, S, S)$

Figure 1. Optimized geometries of all diastereomers. By NOESY analysis coupled with computational studies absolute configurations can be assigned to $C_{\beta}$.

Calculated Coordinates:

\section{$(S, R, S)-\mathbf{1 5} \mathbf{a}$}

\begin{tabular}{|c|c|c|c|c|c|}
\hline \multirow{2}{*}{$\begin{array}{l}\text { Center } \\
\text { Number }\end{array}$} & \multirow{2}{*}{$\begin{array}{l}\text { Atomic } \\
\text { Number }\end{array}$} & \multirow{2}{*}{$\begin{array}{c}\text { Atomic } \\
\text { Type }\end{array}$} & \multicolumn{3}{|c|}{ Coordinates (Angstroms) } \\
\hline & & & $x$ & Y & z \\
\hline 1 & 7 & 0 & 0.772825 & -1.127413 & -0.4341 \\
\hline 2 & 6 & 0 & 1.498619 & -0.040336 & 0.2369 \\
\hline 3 & 6 & 0 & 1.240846 & 1.316665 & -0.4606 \\
\hline 4 & 7 & 0 & 2.315774 & 2.290700 & -0.2144 \\
\hline 5 & 6 & 0 & -0.689598 & -1.128506 & -0.3789 \\
\hline 6 & 6 & 0 & -1.217854 & -2.317026 & -1.2381 \\
\hline 7 & 6 & 0 & -2.740716 & -2.491654 & -1.1449 \\
\hline 8 & 6 & 0 & -0.782842 & -2.156775 & -2.7015 \\
\hline 9 & 6 & 0 & -0.077909 & 1.929410 & 0.0225 \\
\hline 10 & 8 & 0 & -0.428005 & 1.955784 & 1.1879 \\
\hline 11 & 8 & 0 & -0.729721 & 2.534363 & -0.9869 \\
\hline 12 & 6 & 0 & -3.168645 & 2.423182 & -0.7774 \\
\hline 13 & 6 & 0 & -1.928877 & 3.291959 & -0.6526 \\
\hline 14 & 6 & 0 & 2.982502 & -0.426323 & 0.2041 \\
\hline 15 & 9 & 0 & 3.137621 & -1.723326 & 0.59041 \\
\hline 16 & 9 & 0 & 3.530090 & -0.318436 & -1.01956 \\
\hline 17 & 9 & 0 & 3.714175 & 0.309469 & 1.0632 \\
\hline 18 & 6 & 0 & -1.189862 & -1.243247 & 1.0668 \\
\hline 19 & 8 & 0 & -0.611694 & -1.877644 & 1.92840 \\
\hline 20 & 8 & 0 & -2.339598 & -0.578870 & 1.27215 \\
\hline 21 & 6 & 0 & -2.867747 & -0.632508 & 2.61443 \\
\hline 22 & 1 & 0 & 1.083841 & -1.999310 & -0.0118 \\
\hline 23 & 1 & 0 & 1.244 & 0.060108 & 1.30366 \\
\hline 24 & 1 & 0 & 1.189 & 1.139 & -1.537092 \\
\hline 25 & 1 & 0 & 2.322 & $2.560 \varepsilon$ & 0.767200 \\
\hline
\end{tabular}




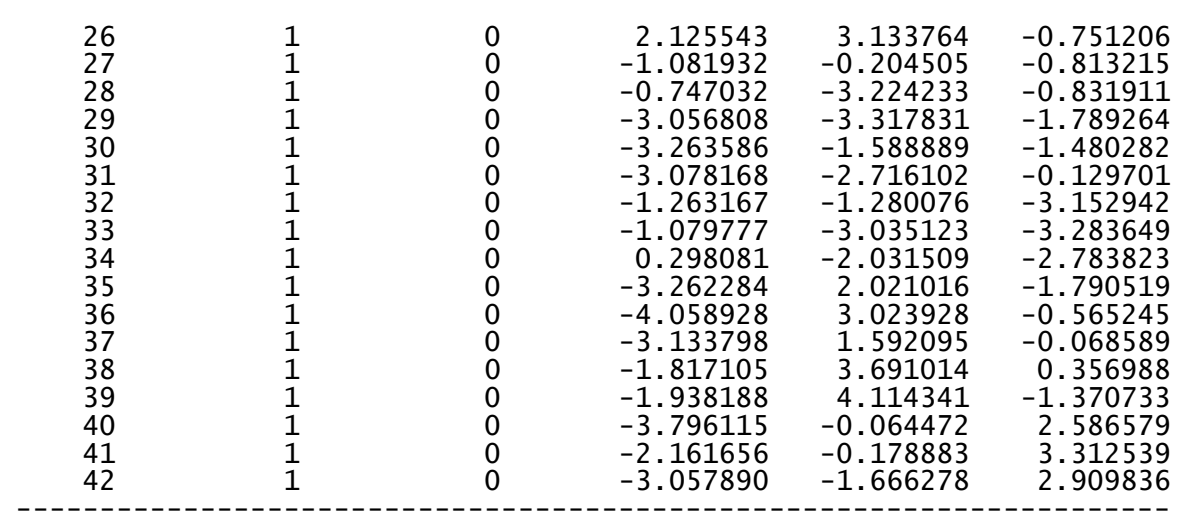

\section{$(S, S, R)-\mathbf{- 1 6} \mathbf{a}$}

\begin{tabular}{|c|c|c|c|c|c|}
\hline $\begin{array}{l}\text { Center } \\
\text { Number }\end{array}$ & $\begin{array}{l}\text { Atomic } \\
\text { Number }\end{array}$ & $\begin{array}{c}\text { Atomic } \\
\text { Type }\end{array}$ & $x^{\text {Coor }}$ & inates ${ }_{Y}$ (Ans & roms) \\
\hline $\begin{array}{r}1 \\
2 \\
3 \\
4 \\
5 \\
6 \\
7 \\
8 \\
9 \\
10 \\
11 \\
12 \\
13 \\
14 \\
15 \\
16 \\
17 \\
18 \\
19 \\
20 \\
21 \\
22 \\
23 \\
24 \\
25 \\
26 \\
27 \\
28 \\
29 \\
30 \\
31 \\
32 \\
33 \\
34 \\
35 \\
36 \\
37 \\
38 \\
39 \\
40 \\
41 \\
42\end{array}$ & $\begin{array}{l}7 \\
6 \\
6 \\
6 \\
1 \\
7 \\
6 \\
1 \\
6 \\
6 \\
1 \\
6 \\
6 \\
9 \\
9 \\
9 \\
8 \\
8 \\
6 \\
1 \\
6 \\
1 \\
1 \\
1 \\
1 \\
1 \\
1 \\
1 \\
1 \\
6 \\
1 \\
1 \\
1 \\
1 \\
1 \\
8 \\
8 \\
6 \\
1 \\
1 \\
\end{array}$ & $\begin{array}{l}0 \\
0 \\
0 \\
0 \\
0 \\
0 \\
0 \\
0 \\
0 \\
0 \\
0 \\
0 \\
0 \\
0 \\
0 \\
0 \\
0 \\
0 \\
0 \\
0 \\
0 \\
0 \\
0 \\
0 \\
0 \\
0 \\
0 \\
0 \\
0 \\
0 \\
0 \\
0 \\
0 \\
0 \\
0 \\
0 \\
0 \\
0 \\
0 \\
0 \\
0 \\
0\end{array}$ & $\begin{array}{r}-2.371778 \\
-1.710654 \\
-0.269493 \\
-2.581808 \\
-1.617214 \\
0.379058 \\
0.534008 \\
-0.349820 \\
1.533051 \\
1.472499 \\
0.403090 \\
2.182038 \\
1.986243 \\
0.437398 \\
0.122850 \\
1.850704 \\
-3.872294 \\
-2.175336 \\
-4.815798 \\
-4.715169 \\
-6.201585 \\
-4.539696 \\
-6.452166 \\
-6.277862 \\
-6.936985 \\
-0.336763 \\
-3.339521 \\
-1.947772 \\
1.494009 \\
2.889482 \\
1.503589 \\
1.796288 \\
3.061419 \\
1.769298 \\
3.252670 \\
2.060641 \\
2.831042 \\
3.929691 \\
4.078841 \\
4.457898 \\
4.824322 \\
3.854442\end{array}$ & $\begin{array}{r}-1.762194 \\
-0.856719 \\
-0.481024 \\
0.401430 \\
-1.269466 \\
0.261064 \\
-1.753658 \\
0.066110 \\
1.147658 \\
2.045547 \\
2.263381 \\
3.388873 \\
1.420673 \\
-2.668349 \\
-2.355571 \\
-1.489179 \\
0.072338 \\
1.548404 \\
1.169561 \\
1.843206 \\
0.566364 \\
1.721674 \\
0.012499 \\
-0.110615 \\
1.365949 \\
0.854280 \\
-1.870399 \\
-2.683572 \\
1.848890 \\
0.465340 \\
0.473609 \\
2.111706 \\
1.239667 \\
3.904076 \\
3.239140 \\
4.051291 \\
-0.470989 \\
0.791915 \\
-1.116626 \\
-1.653005 \\
-0.385895 \\
-1.814574\end{array}$ & $\begin{array}{r}0.842789 \\
-0.101916 \\
0.350334 \\
-0.246148 \\
-1.122233 \\
-0.731975 \\
0.653090 \\
1.299078 \\
-0.469436 \\
0.808494 \\
0.930860 \\
0.560147 \\
2.118865 \\
-0.337054 \\
1.792609 \\
0.826973 \\
-0.372358 \\
-0.285233 \\
-0.541822 \\
0.313045 \\
-0.637672 \\
-1.444247 \\
0.271264 \\
-1.493000 \\
-0.765996 \\
-1.133264 \\
0.553547 \\
0.792467 \\
-1.315240 \\
-0.700124 \\
2.366315 \\
2.947238 \\
2.065212 \\
-0.314547 \\
0.396369 \\
1.423690 \\
-1.662420 \\
-0.160125 \\
-1.972926 \\
-1.099845 \\
-2.294715 \\
-2.778672\end{array}$ \\
\hline
\end{tabular}

$(S, R, R)$

\begin{tabular}{|c|c|c|c|c|c|}
\hline $\begin{array}{l}\text { Center } \\
\text { Number }\end{array}$ & $\begin{array}{l}\text { Atomic } \\
\text { Number }\end{array}$ & $\begin{array}{l}\text { Atomic } \\
\text { Type }\end{array}$ & $x^{\operatorname{CoO}}$ & $\operatorname{nates}_{Y}$ (An & roms) \\
\hline $\begin{array}{r}1 \\
2 \\
3 \\
4 \\
5 \\
6 \\
7 \\
8 \\
9 \\
10 \\
11 \\
12\end{array}$ & $\begin{array}{l}7 \\
6 \\
6 \\
6 \\
6 \\
1 \\
1 \\
6 \\
6 \\
1 \\
7 \\
9\end{array}$ & $\begin{array}{l}0 \\
0 \\
0 \\
0 \\
0 \\
0 \\
0 \\
0 \\
0 \\
0 \\
0 \\
0\end{array}$ & $\begin{array}{r}-0.771636 \\
0.191325 \\
-2.185602 \\
-2.257368 \\
-3.048154 \\
-2.620936 \\
-0.671504 \\
1.525652 \\
0.477771 \\
-0.191279 \\
1.328742 \\
0.633878\end{array}$ & $\begin{array}{r}-0.748857 \\
-0.568683 \\
-0.351479 \\
1.177034 \\
-1.078039 \\
-0.564753 \\
-1.643912 \\
0.019746 \\
-1.876397 \\
0.142666 \\
1.087718 \\
-2.909617\end{array}$ & $\begin{array}{r}-1.135217 \\
-0.061922 \\
-1.014701 \\
-0.906615 \\
0.051734 \\
-1.996543 \\
-1.597920 \\
-0.639529 \\
0.683003 \\
0.670192 \\
-1.620362 \\
-0.189130\end{array}$ \\
\hline
\end{tabular}




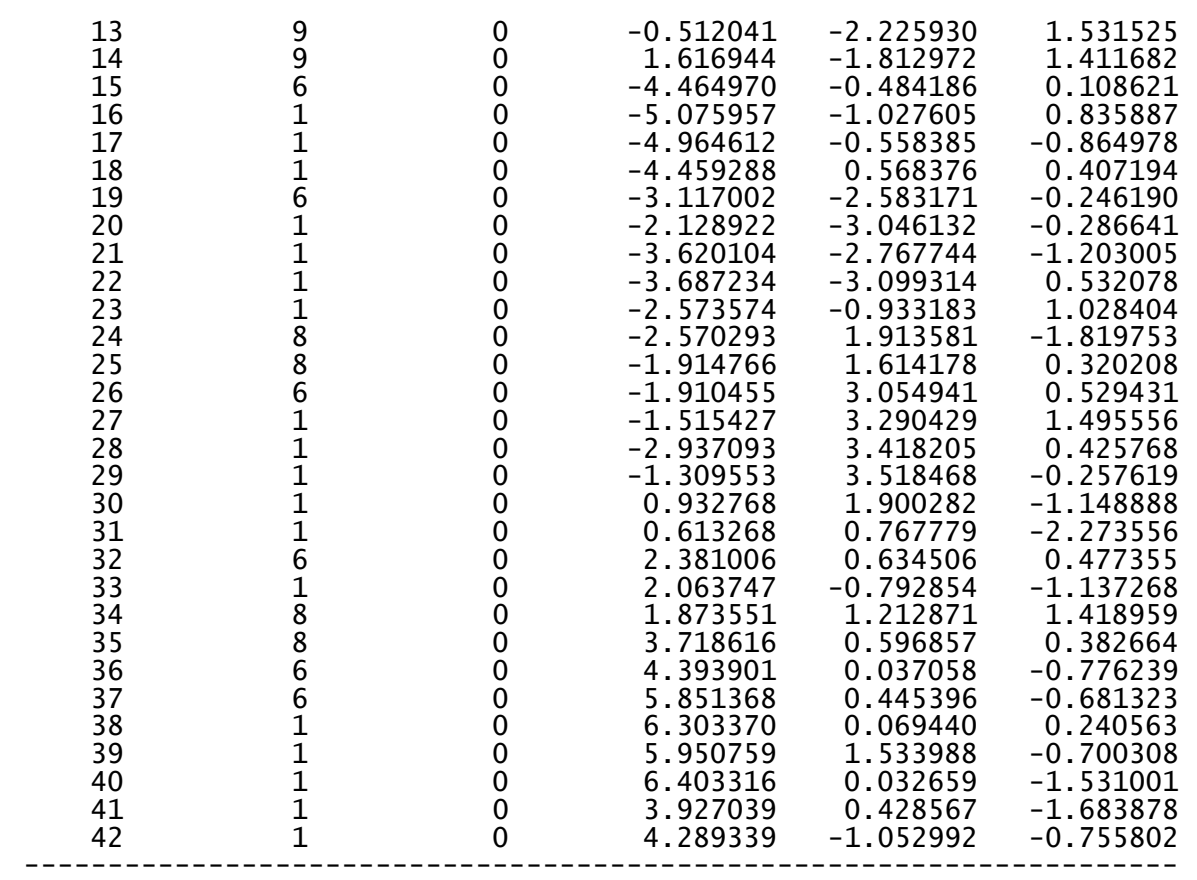

\section{$(S, S, S)$}

\begin{tabular}{|c|c|c|c|c|c|}
\hline \multirow{2}{*}{$\begin{array}{l}\text { Center } \\
\text { Number }\end{array}$} & \multirow{2}{*}{$\begin{array}{l}\text { Atomic } \\
\text { Number }\end{array}$} & \multirow{2}{*}{$\begin{array}{c}\text { Atomic } \\
\text { Type }\end{array}$} & \multicolumn{3}{|c|}{ Coordinates (Angstroms) } \\
\hline & & & $\mathrm{x}$ & & z \\
\hline 1 & 7 & 0 & 0.724845 & -0.659200 & -0.6139 \\
\hline 2 & 6 & 0 & -0.284848 & -1.098686 & 0.3407 \\
\hline 3 & 6 & 0 & -1.594748 & -1.551275 & -0.3940 \\
\hline 4 & 7 & 0 & -1.461171 & -2.549160 & -1.4431 \\
\hline 5 & 6 & 0 & 1.694412 & 0.335747 & -0.1516 \\
\hline 6 & 6 & 0 & 2.734601 & 0.598438 & -1.2776 \\
\hline 7 & 6 & 0 & 3.707142 & 1.736504 & -0.9351 \\
\hline 8 & 6 & 0 & 3.502290 & -0.689918 & -1.6055 \\
\hline 9 & 6 & 0 & 0.996585 & 1.639342 & 0.2599 \\
\hline 10 & 8 & 0 & 0.022976 & 2.101001 & -0.3057 \\
\hline 11 & 8 & 0 & 1.585924 & 2.215902 & 1.3209 \\
\hline 12 & 6 & 0 & 1.024748 & 3.473830 & 1.751 \\
\hline 13 & 6 & 0 & -2.224547 & -0.298724 & -1.0045 \\
\hline 14 & 8 & 0 & -2.103483 & 0.006660 & -2.1760 \\
\hline 15 & 8 & 0 & -2.869759 & 0.427695 & -0.0891 \\
\hline 16 & 6 & 0 & -4.067587 & 2.362748 & 0.65621 \\
\hline 17 & 6 & 0 & -3.388988 & 1.715009 & -0.5329 \\
\hline 18 & 6 & 0 & 0.258689 & -2.233308 & 1.2125 \\
\hline 19 & 9 & 0 & -0.677826 & -2.645824 & 2.0979 \\
\hline 20 & 9 & 0 & 1.335613 & -1.840775 & 1.933 \\
\hline 21 & 9 & 0 & 0.641519 & -3.317790 & 0.5002 \\
\hline 22 & 1 & 0 & 0.260072 & -0.291427 & -1.4412 \\
\hline 23 & 1 & 0 & -0.583179 & -0.316118 & 1.0566 \\
\hline 24 & 1 & 0 & -2.269019 & -1.921362 & 0.38401 \\
\hline 25 & 1 & 0 & -0.818620 & -3.280164 & -1.1574 \\
\hline 26 & 1 & 0 & -1.083993 & -2.112533 & -2.280 \\
\hline 27 & 1 & 0 & 2.218617 & -0.054118 & 0.723 \\
\hline 28 & 1 & 0 & 2.161091 & 0.899660 & -2.166 \\
\hline 29 & 1 & 0 & 4.256197 & 1.522943 & -0.011 \\
\hline 30 & 1 & 0 & 3.201025 & 2.697873 & -0.8097 \\
\hline 31 & 1 & 0 & 4.439796 & 1.852776 & -1.7396 \\
\hline $3 \overline{2}$ & 1 & 0 & 2.819832 & -1.510540 & -1.83420 \\
\hline 33 & 1 & 0 & 4.161591 & -0.531746 & -2.46501 \\
\hline 34 & 1 & 0 & 4.126212 & -0.993735 & -0.75653 \\
\hline 35 & 1 & 0 & 1.091792 & 4.214858 & 0.9526 \\
\hline 36 & 1 & 0 & -0.021392 & 3.345126 & 2.037 \\
\hline 37 & 1 & 0 & 1.620100 & 3.781635 & 2.609 \\
\hline 38 & 1 & 0 & -3.357276 & 2.521107 & 1.472 \\
\hline 39 & 1 & 0 & -4.472077 & 3.335091 & 0.359 \\
\hline 40 & 1 & 0 & -4.891764 & 1.745663 & \\
\hline 4. & 1 & 0 & -2.547476 & 2.305063 & -0.90234 \\
\hline 42 & 1 & 0 & -4.082751 & 1.539678 & $-1.3594 \mathrm{C}$ \\
\hline
\end{tabular}




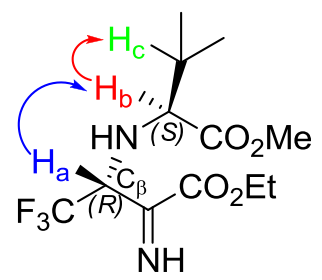

$17 \mathrm{a}$

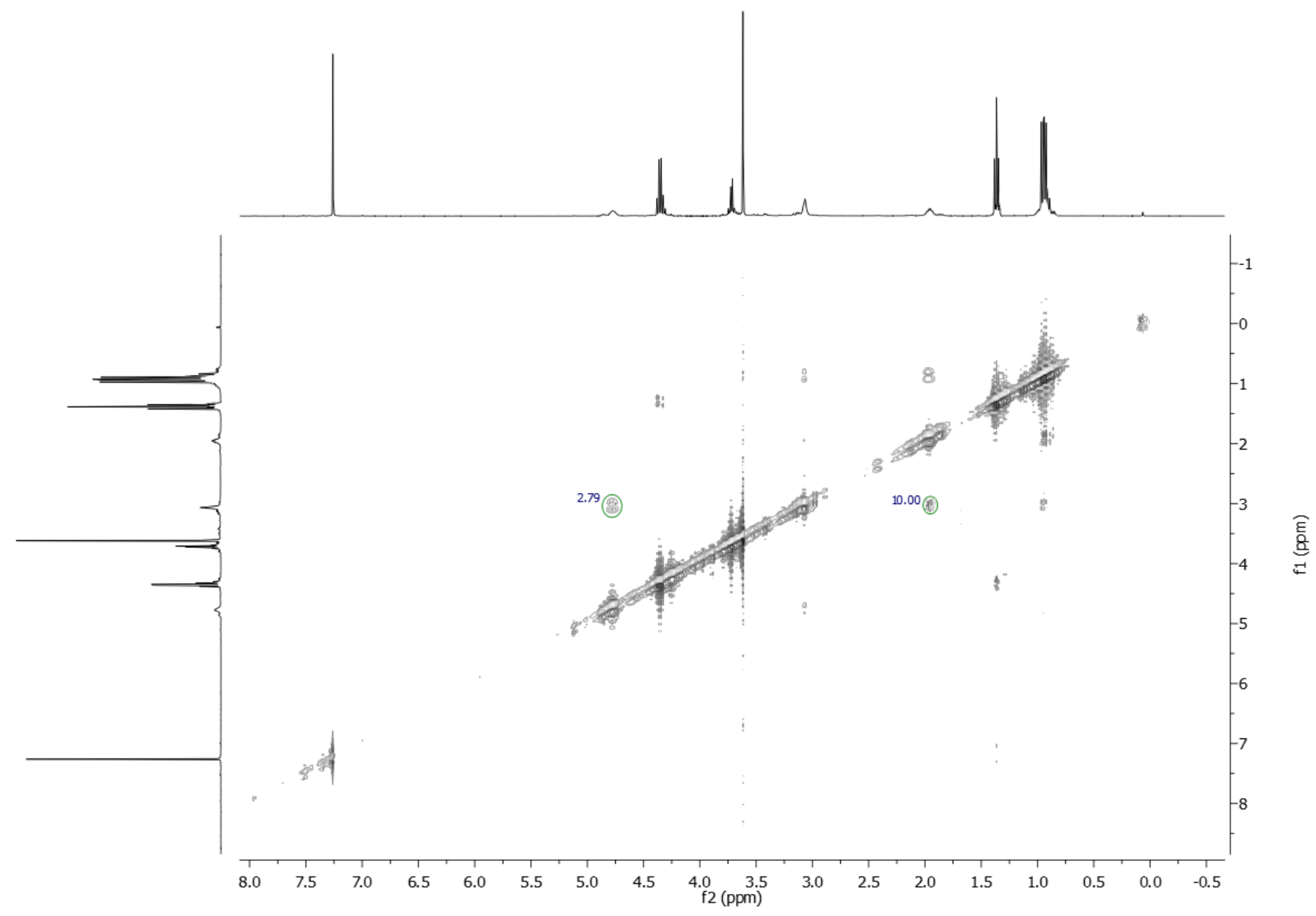

NOESY spectrum of 17a. The cross peak between the protons $\mathrm{H}_{\mathrm{b}}$ and $\mathrm{H}_{\mathrm{c}}$ was used as a distance ruler and its volume was set to 10.00 a.u.; the cross peak between $\mathrm{H}_{\mathrm{d}}$ and $\mathrm{H}_{\mathrm{e}}$ was measured $0.83 \mathrm{a}$. u. (corresponding to an interproton distance of $3.74 \AA$ ), in order to determine the absolute configuration.

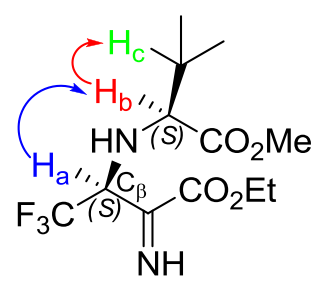

18a 


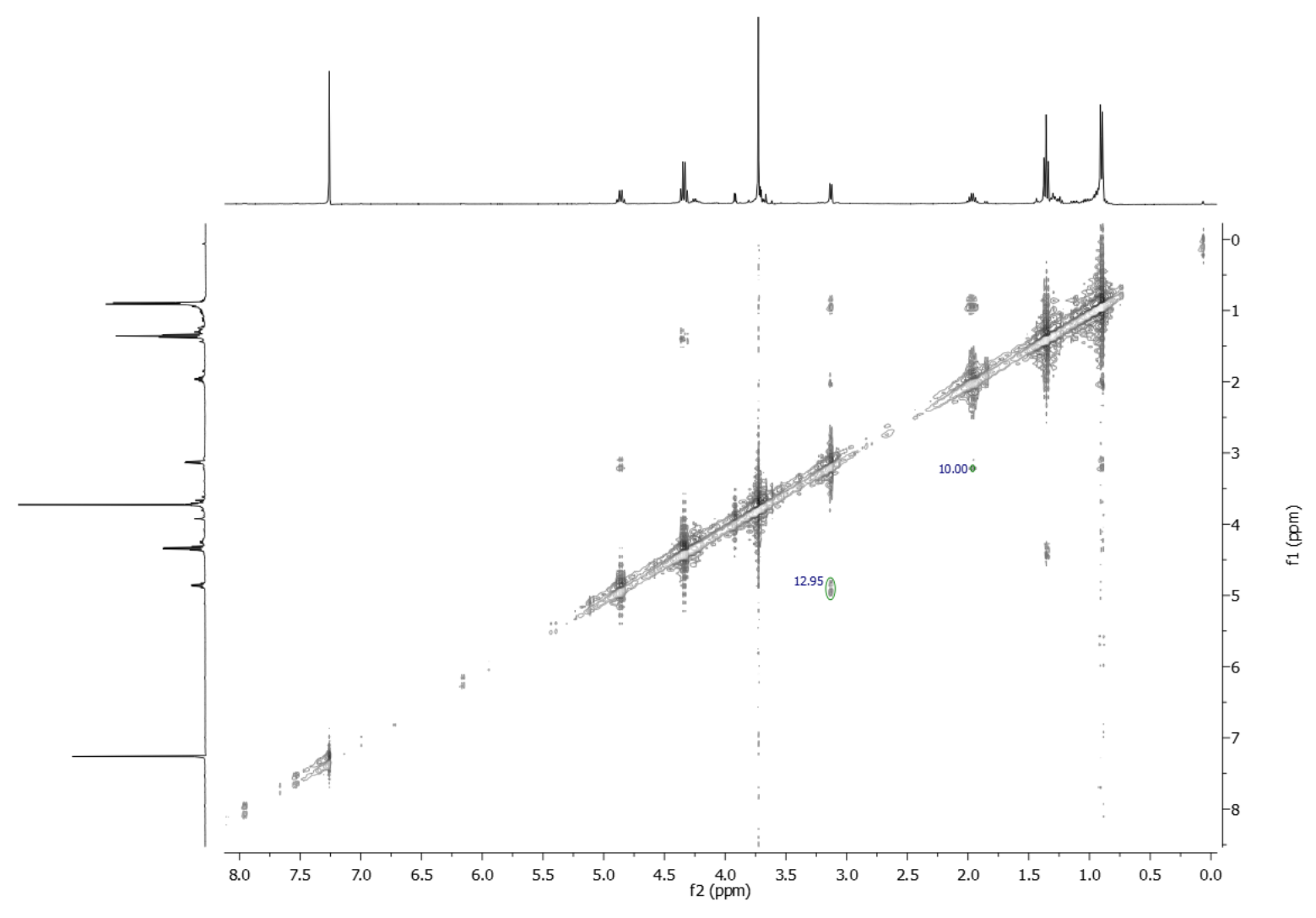

NOESY spectrum of 18a. The cross peak between the protons $\mathrm{H}_{\mathrm{b}}$ and $\mathrm{H}_{\mathrm{c}}$ was used as a distance ruler and its volume was set to 10.00 a.u.; the cross peak between $H_{d}$ and $H_{e}$ was measured 12.95 a. u. (corresponding to an interproton distance of $2.90 \AA$ ), in order to determine the absolute configuration.

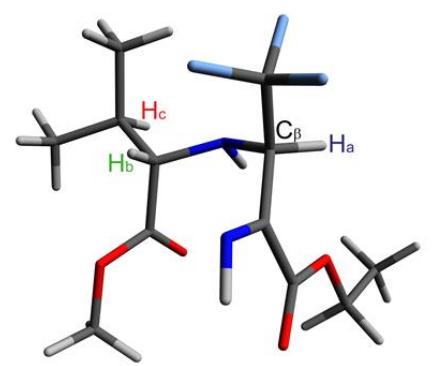

$\mathrm{H}_{\mathrm{a}} / \mathrm{H}_{\mathrm{b}}: 3.671 \AA$

$(S, R)-17 a$

major diastereomer obtained

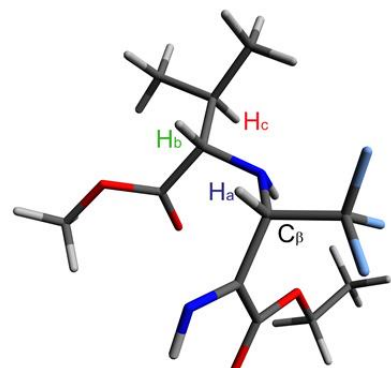

$\mathrm{H}_{\mathrm{a}} / \mathrm{H}_{\mathrm{b}}: 2.279 \AA$

$(S, S)-18 \mathbf{a}$

minor diastereomer obtained

Figure 1. Optimized geometries of all diastereomers. By NOESY analysis coupled with computational studies absolute configurations can be assigned to $\mathrm{C}_{\beta}$.

Calculated Coordinates:

$(S, R)-17 \mathbf{a}$

\begin{tabular}{|c|c|c|c|c|c|}
\hline \multirow{2}{*}{$\begin{array}{l}\text { Center } \\
\text { Number }\end{array}$} & \multirow{2}{*}{$\begin{array}{l}\text { Atomic } \\
\text { Number }\end{array}$} & \multirow{2}{*}{$\begin{array}{l}\text { Atomic } \\
\text { Type }\end{array}$} & \multicolumn{3}{|c|}{ Coordinates (Angstroms) } \\
\hline & & & $\mathrm{X}$ & $\mathrm{Y}$ & Z \\
\hline$\frac{1}{2}$ & $\begin{array}{l}7 \\
6\end{array}$ & $\begin{array}{l}0 \\
0\end{array}$ & $\begin{array}{r}-0.620383 \\
0.007012\end{array}$ & $\begin{array}{l}-0.375900 \\
-1.422894\end{array}$ & $\begin{array}{l}0.946579 \\
0.139432\end{array}$ \\
\hline
\end{tabular}




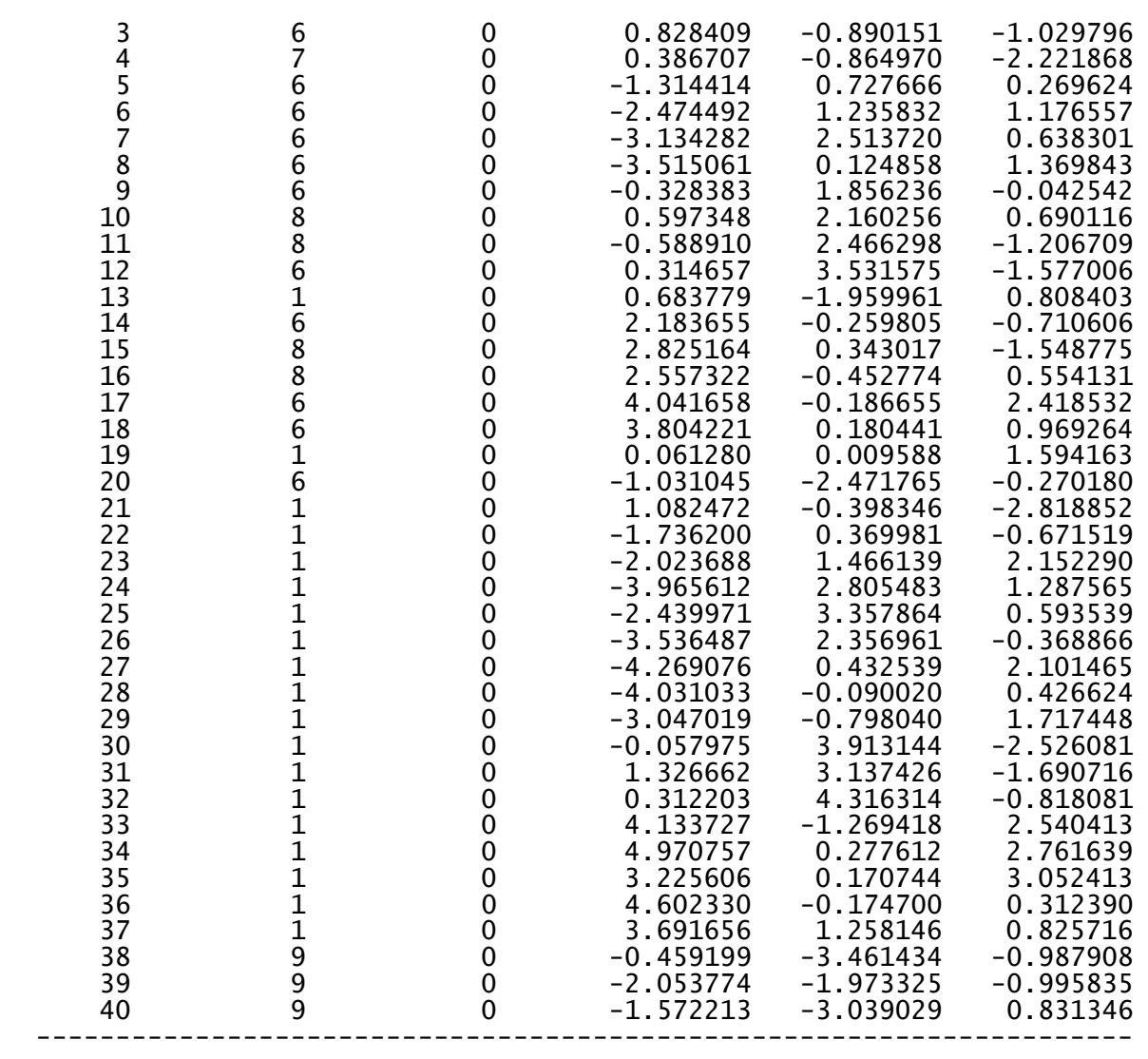

\section{$(S, S)$-18a}

\begin{tabular}{|c|c|c|c|c|c|}
\hline \multirow{2}{*}{$\begin{array}{l}\text { Center } \\
\text { Number }\end{array}$} & \multirow{2}{*}{$\begin{array}{l}\text { Atomic } \\
\text { Number }\end{array}$} & \multirow{2}{*}{$\begin{array}{l}\text { Atomic } \\
\text { Type }\end{array}$} & \multicolumn{3}{|c|}{ Coordinates (Angstroms) } \\
\hline & & & $\mathrm{x}$ & $\mathrm{Y}$ & $z$ \\
\hline 1 & 7 & 0 & 0.345116 & 0.964983 & 0.1884 \\
\hline 2 & 6 & 0 & -0.546248 & 0.834902 & -0.9526 \\
\hline 3 & 6 & 0 & -0.984384 & -0.586300 & -1.3256 \\
\hline 4 & 7 & 0 & -0.713287 & -1.017381 & -2.4920 \\
\hline 5 & 6 & 0 & 1.749213 & 0.620516 & -0.0220 \\
\hline 6 & 6 & 0 & 2.606085 & 1.241529 & 1.1208 \\
\hline 7 & 6 & 0 & 4.070941 & 0.782213 & 1.0841 \\
\hline 8 & 6 & 0 & 2.512900 & 2.772585 & 1.0797 \\
\hline 9 & 6 & 0 & 1.953911 & -0.897471 & -0.0820 \\
\hline 10 & 8 & 0 & 1.353658 & -1.687038 & 0.6246 \\
\hline 11 & 8 & 0 & 2.871868 & -1.255624 & -0.9918 \\
\hline 12 & 6 & 0 & 3.146839 & -2.669945 & -1.0905 \\
\hline 13 & 6 & 0 & -1.721081 & 1.809049 & -0.7743 \\
\hline 14 & 6 & 0 & -1.665756 & -1.519624 & -0.3281 \\
\hline 15 & 8 & 0 & -1.998765 & -2.647785 & -0.6312 \\
\hline 16 & 8 & 0 & -1.852427 & -0.959797 & 0.8662 \\
\hline 17 & 6 & 0 & -2.642958 & -0.928005 & 3.1268 \\
\hline 18 & 6 & 0 & -2.499180 & -1.779926 & 1.8835 \\
\hline 19 & 1 & 0 & -0.026748 & 0.459917 & \\
\hline 20 & 1 & 0 & -1.040433 & -1.990366 & -2.5659 \\
\hline 21 & 1 & 0 & 2.075745 & 1.047768 & -0.9760 \\
\hline 22 & 1 & 0 & 2.162464 & 0.888601 & 2.0628 \\
\hline 23 & 1 & 0 & 4.634597 & 1.276351 & 1.8814 \\
\hline 24 & 1 & 0 & 4.175362 & -0.297096 & 1.2281 \\
\hline 25 & 1 & 0 & 4.543855 & 1.041686 & 0.1304 \\
\hline 26 & 1 & 0 & 3.002760 & 3.208641 & 1.9562 \\
\hline 27 & $\overline{1}$ & 0 & 3.014939 & 3.165826 & 0.1874 \\
\hline 28 & 1 & 0 & 1.472626 & 3.103357 & 1.05 \\
\hline 29 & 1 & 0 & 3.901356 & -2.767194 & -1.8693 \\
\hline 30 & 1 & 0 & 2.239843 & -3.212591 & -1.3638 \\
\hline 31 & 1 & 0 & 3.523918 & -3.051916 & -0.1396 \\
\hline 32 & 1 & 0 & -3.234273 & -0.032349 & 2.9187 \\
\hline 33 & 1 & 0 & -3.150028 & -1.504856 & 3.9056 \\
\hline 34 & $\overline{1}$ & 0 & -1.665298 & -0.621526 & 3.50 \\
\hline 35 & 1 & 0 & -3.464539 & -2.108517 & 1.48 \\
\hline 36 & 1 & 0 & -1.877509 & -2.663427 & 2.0505 \\
\hline 37 & $\overline{9}$ & 0 & -2.685241 & 1.571994 & -1.692740 \\
\hline 38 & 9 & 0 & -1.303183 & 3.080514 & -0.9433 \\
\hline & 9 & 0 & -2.296131 & 1.741904 & 0.442 \\
\hline 40 & 1 & 0 & -0.026807 & 1.199579 & -1.843195 \\
\hline
\end{tabular}

